\title{
IMPACT OF NONCOVALENT INTERACTIONS ON THE STRUCTURAL CHEMISTRY OF THORIUM(IV)-AQUO-CHLORO COMPLEXES
}

Jennifer N. Wacker, ${ }^{1}$ Aaron D. Nicholas, ${ }^{2}$ Monica Vasiliu, ${ }^{3}$ Alexander C. Marwitz, ${ }^{1}$ Jeffery A. Bertke, ${ }^{1}$ David A. Dixon, ${ }^{2}$ and Karah E. Knope ${ }^{1, *}$

1 Department of Chemistry, Georgetown University, Washington, D.C. 20057, United States of America

2 Department of Chemistry, The George Washington University, Washington, D.C. 20052, United States of America 3 Department of Chemistry and Biochemistry, The University of Alabama, Tuscaloosa, Alabama 35487, United States of America

*kek44@georgetown.edu

\section{ELECTRONIC SUPPLEMENTAL INFORMATION}

$\underline{\text { Table of Contents }}$

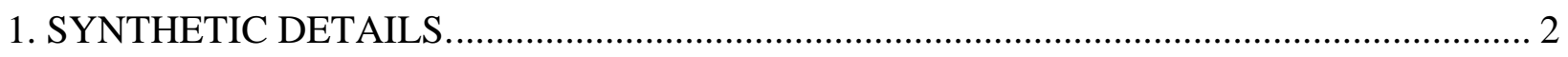

2. CRYSTALLOGRAPHIC REFINEMENT DETAILS OF COMPOUNDS 1-5 ........................ 6

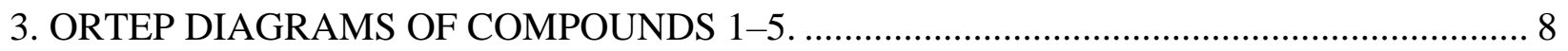

4. POWDER X-RAY DIFFRACTION PLOTS OF COMPOUNDS 3-4 ................................... 11

5. RAMAN AND INFRARED PLOTS OF COMPOUNDS 1-5 .............................................. 13

6. SUPRAMOLECULAR INTERACTIONS OBSERVED IN COMPOUNDS 1-5 .................. 18

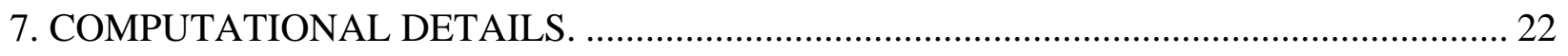

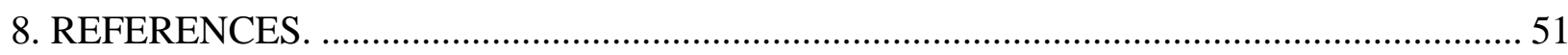




\section{SYNTHETIC DETAILS.}

\section{Synthesis of Bis(4-hydroxy-3,4-dihydroquinazolin-1-ium) Thorium(IV) Diaquo}

Hexachloride Monohydrate, $(\mathbf{Q u i n o H})_{2}\left[\mathbf{T h}\left(\mathbf{H}_{2} \mathbf{O}\right)_{2} \mathbf{C l}_{6}\right] \cdot \mathbf{H}_{2} \mathbf{O} ; \mathbf{1}$. ThCl $4(0.049 \mathrm{~g}, 0.132 \mathrm{mmol})$ was dissolved in hydrochloric acid $\left(\mathrm{HCl}_{(a q)}, 500 \mu \mathrm{L}, 3 \mathrm{M}\right)$ to generate an acidic aqueous solution of $\mathrm{Th}^{\mathrm{IV}}(0.262 \mathrm{M})$. The vial was subsequently charged with quinazoline (17.2 $\left.\mathrm{mg}, 0.132 \mathrm{mmol}\right)$ to yield a yellow solution, which was left to evaporate under a nitrogen atmosphere. After approximately 7 days, yellow/orange block crystals formed in an oil-like layer at the bottom of the vial. Single crystal X-ray diffraction determined the crystals to be $(\text { QuinoH })_{2}\left[\mathrm{Th}\left(\mathrm{H}_{2} \mathrm{O}\right)_{2} \mathrm{Cl}_{6}\right] \cdot \mathrm{H}_{2} \mathrm{O}$ (1). The bulk phase, however, dissolves readily under ambient conditions. Due to the sensitivity of this compound, further analyses to assess both the yield and phase purity of this reaction could not feasibly be performed. Therefore, yield determination, powder X-ray diffraction, and elemental analysis are not reported. Further, excitation of the QuinoH counter-ions led to fluorescence that overwhelmed vibrational transitions, so only the IR data are reported. IR $\left(\mathrm{cm}^{-1}\right): 402(\mathrm{~s}), 455(\mathrm{~m}$, sh), 468 (s), 511 (m), 579 (m), 639 (m), 745 (m, sh), 761 (s), 792 (m), 804 (m), 872 (w), 935 (m, sh), 953 (m), 974 (m), 985 (s), 1010 (vs), 1017 (s, sh), 1042 (w, b), 1120 (m, b), 1157 (m, b), 1215 (vs), 1235 (w), 1270 (m, b), 1286 (m, b), 1319 (m), 1345 (w), 1376 (w, sh), 1435 (s), 1472 (m, sh), 1495 (s), 1569 (vs), 1597 (m), 1621 (s), 1652 (m), 1662 (s), 2838 (s, sh), 2991 (s, sh), 3052 (vs, sh, b), 3122 (vs, b), 3205 (vs, b), 3247 (vs, b).

\section{Synthesis of Thorium(IV) Tetraaquo Tetrachloro Bis(2-Methylpyridinium) Dichloride} Hydrate, $\left[\mathbf{T h}\left(\mathbf{H}_{2} \mathbf{O}\right)_{4} \mathbf{C l}_{4}\right] \cdot 2(2-\mathrm{MePyH} \cdot \mathbf{C l}) \cdot \mathbf{H}_{2} \mathrm{O} ; 2$. $\mathrm{ThCl}_{4}(0.049 \mathrm{~g}, 0.132 \mathrm{mmol})$ was dissolved in hydrochloric acid $\left(\mathrm{HCl}_{(a q)}, 500 \mu \mathrm{L}, 3 \mathrm{M}\right)$ to generate an acidic aqueous solution of $\mathrm{Th}^{\mathrm{IV}}(0.262 \mathrm{M})$. The vial was subsequently charged with 2-methylpyridine (13.04 $\mu \mathrm{L}, 0.132 \mathrm{mmol})$ to yield a colorless solution, which was left to evaporate under a nitrogen atmosphere. After approximately 7 days, colorless block crystals formed at the bottom of the vial. Single crystal X-ray diffraction determined the crystals to be $\left[\mathrm{Th}\left(\mathrm{H}_{2} \mathrm{O}\right)_{4} \mathrm{Cl}_{4}\right] \cdot 2(2-\mathrm{MePyH} \cdot \mathrm{Cl}) \cdot \mathrm{H}_{2} \mathrm{O}(2)$. The bulk phase, however, dissolves readily under ambient conditions. Due to the sensitivity of this compound, further analyses to assess both the yield and phase purity of this reaction could not be feasibly performed. Therefore, yield determination, powder X-ray diffraction, and elemental analysis are not reported. IR $\left(\mathrm{cm}^{-1}\right): 453.8$ (w, sh), $468.8(\mathrm{vs}), 540.0(\mathrm{~m}), 596.3(\mathrm{~m}, \mathrm{~b}), 626.3(\mathrm{~s}), 645.0(\mathrm{~m}, \mathrm{~b}), 705.0$ (s), 
761.3 (vs), 806.3 (w), 875.7 (w), 937.5 (m, b), 988.2 (m, b), 1016. 8 (w), 1026.2 (w), 1045.0 (m), 1098.1 (w, sh), 1107.5 (m), 1166.8 (m), 1235. 6 (m), 1254.3 (w, sh), 1288.7 (s), 1382.5 (w, sh), 1395.0 (m), 1476.2 (s), 1538.7 (s), 1557.5 (w), 1613.7 (vs), 1632.5 (s), 2653.5 (s, b), 2759.0 (s, b), 2860.3 (s, b), 2939.0 (s, b), 3062.8 (s, b), 3152.8 (s, sh), 3254.0 (s, sh), 3434.0 (m, sh), 3557.7 (w). Raman ( $\left.\mathrm{cm}^{-1}\right)$ : $163.5(\mathrm{w}), 216.1(\mathrm{w}), 230.2$ (w), $258.3(\mathrm{w}), 544.2(\mathrm{w}), 628.4(\mathrm{w}), 633.1$ (w, sh), 715.9 (w, b), 808.1 (m), 1017.4 (m), 1047.1 (w), 1108.4 (w), 1167.5 (w), $1240.6(\mathrm{~m}), 1251.9(\mathrm{~m})$, $1296.9(\mathrm{w}), 1381.3$ (m), 1395.3 (w), 1443.1 (m, b), 1541.6 (w), 1617.5 (m), 1634.4 (s), 1648.4 (m, sh), 2189.4 (w, b), 2848.6 (vs, sh), 2873.6 (vs, sh), 2898.6 (vs, sh), 2923.6 (vs), 2989.1 (m, sh), 3040.7 (m, b), 3064.7 (w, sh), 3088.89 (m, sh).

\section{Synthesis of Thorium(IV) Tetraaquo Tetrachloro Bisphenanthrolinium Dichloride,} $\left[\mathbf{T h}\left(\mathrm{H}_{2} \mathrm{O}\right)_{4} \mathbf{C l}_{4}\right] \cdot \mathbf{2}(\mathbf{P h e n H} \cdot \mathbf{C l}) \cdot \mathbf{2} \mathrm{H}_{2} \mathrm{O} ; 3 . \mathrm{ThCl}_{4}(0.049 \mathrm{~g}, 0.132 \mathrm{mmol})$ was dissolved in hydrochloric acid $\left(\mathrm{HCl}_{(a q)}, 500 \mu \mathrm{L}, 3 \mathrm{M}\right)$ to generate an acidic aqueous solution of $\mathrm{Th}^{\mathrm{IV}}(0.262 \mathrm{M})$. The vial was subsequently charged with 1,10-phenanthroline $(23.8 \mathrm{mg}, 0.132 \mathrm{mmol})$ to yield a colorless solution, which was left to evaporate under a nitrogen atmosphere. After approximately 7 days, colorless needle crystals formed at the bottom and side of the vial. Single crystal X-ray diffraction determined the crystals to be $\left[\mathrm{Th}\left(\mathrm{H}_{2} \mathrm{O}\right)_{4} \mathrm{Cl}_{4}\right] \cdot 2(\mathrm{PhenH} \cdot \mathrm{Cl}) \cdot 2 \mathrm{H}_{2} \mathrm{O}(3)$. The bulk product was washed with water and allowed to dry under a nitrogen atmosphere. Approximate yield based on thorium: $0.058 \mathrm{~g}, 47.5 \%$. Powder X-ray diffraction (Figure S6) confirmed that the single crystal used for structure determination was representative of the bulk product. IR $\left(\mathrm{cm}^{-1}\right): 406.3(\mathrm{~m})$, $412.0(\mathrm{~m}), 417.6(\mathrm{~m}), 462.6(\mathrm{~s}), 494.0(\mathrm{w}), 536.4(\mathrm{~m}), 551.9(\mathrm{w}), 620.7(\mathrm{~s}), 640.3$ (w, b), 714.7 (vs), 745.4 (w), 777.9 (vs), 817.8 (vs), 843.9 (vs), 853.9 (m, sh), 883.8 (m), 897.3 (w), 955.1 (w), 975.0 (w), 986.3 (w), 1004.4 (w), 1010.7 (w), 1016.9 (w), 1026.3 (w), 1034.1 (w), $1045.1(w)$, 1070.1 (w), 1077.9 (w, sh), 1082.6 (w), 1095.1 (m), 1138.8 (m), 1143.5 (w), 1146.6 (m), 1151.3 (w), 1185.7 (s), 1191.9 (w), 1201.3 (w), 1213.8 (w), 1224.8 (w, sh), 1229.4 (m), 1238.8 (m), 1245.1 (w, sh), 1279.4 (m), 1287.3 (m), 1299.8 (w), 1316.9 (m), 1335.7 (m), 1346.6 (w), 1356.0 (w), $1365.4(\mathrm{w}), 1373.2$ (w), 1404.4 (w), 1416.9 (m), 1423.2 (m), 1432.6 (w), 1449.8 (s), 1465.4 (s), 1493.5 (s), $1501.4(\mathrm{w}), 1520.2$ (m, sh), 1535.8 (vs), 1563.9 (w), $1584.3(\mathrm{~m}), 1595.2(\mathrm{~s}), 1615.5$ (s), 1634.3 (m), 1649.9 (w, b), 2854.6 (m, sh), 2925.2 (m), 2956.5 (m), 2994.0 (w), 3034.2 (s, sh), 3056.7 (s), 3075.5 (s), 3098.0 (s), 3210.5 (s, b), 3319.2 (s, b), 3458 (m, sh, b). Raman (cm ${ }^{-1}$ ): $128.0(w), 142.1(w), 167.1(w), 226.4(\mathrm{~m}), 240.5(w, s h), 246.8(w), 259.3(w), 398.3(w), 412.4$ 
(s), $462.4(w), 509.3(w), 551.4(w), 598.3(w), 712.0(\mathrm{~s}), 719.6(w, s h), 852.9(w), 884.7(w)$, $974.0(\mathrm{w}), 985.0(\mathrm{w}), 1026.3(\mathrm{w}), 1043.5(\mathrm{~m}), 1077.0(\mathrm{w}), 1095.8(\mathrm{w}), 1142.2(\mathrm{w}), 1195.6(\mathrm{w})$, $1229.4(\mathrm{w}), 1246.3$ (w), 1277.2 (w, sh), 1291.3 (m), 1316.6 (m), 1344.7 (w), 1372.8 (s), 1415.0 (vs), 1448.8 (vs), 1471.3 (s), 1496.6 (w), 1544.4 (m), 1583.8 (m), 1597.8 (m), 1611.9 (m), 1631.6 (m), 1645.6 (w, sh), 3036.3 (m, sh), 3055.0 (s), 3073.8 (s), 3092.5 (s, sh), 3122.6 (m, sh).

\section{Synthesis of Thorium(IV) Tetraaquo Tetrachloro Bis(Terpyridinium) Tetrachloride} Trihydrate, $\left[\mathbf{T h}\left(\mathbf{H}_{2} \mathbf{O}\right)_{4} \mathbf{C l}_{4}\right] \cdot \mathbf{2}\left(\operatorname{TerpyH}_{2} \cdot \mathbf{2} \mathbf{C l}\right) \cdot \mathbf{3} \mathbf{H}_{2} \mathbf{O} ; 4$. $\mathrm{ThCl}_{4}(0.049 \mathrm{~g}, 0.132 \mathrm{mmol})$ was dissolved in hydrochloric acid $\left(\mathrm{HCl}_{(a q)}, 500 \mu \mathrm{L}, 3 \mathrm{M}\right)$ to generate an acidic aqueous solution of $\operatorname{Th}^{\text {IV }}(0.262 \mathrm{M})$. The vial was subsequently charged with 2,2':6',2"'-terpyridine (15.4 mg, 0.066 mmol) to yield a yellow solution, which was left to evaporate under a nitrogen atmosphere. After approximately 7 days, colorless rods formed at the bottom and side of the vial. Single crystal Xray diffraction determined the crystals to be $\left[\mathrm{Th}\left(\mathrm{H}_{2} \mathrm{O}\right)_{4} \mathrm{Cl}_{4}\right] \cdot 2\left(\mathrm{TerpyH}_{2} \cdot 2 \mathrm{Cl}\right) \cdot 3 \mathrm{H}_{2} \mathrm{O}(4)$. Although a 1:1 Th:terpy ratio was performed and yielded 4 , better crystallinity was attained when a ratio of 1:0.5 was used. The latter ratio is the synthetic method reported. The bulk product was washed with water and allowed to dry under a nitrogen atmosphere. Approximate yield based on thorium: $0.046 \mathrm{~g}, 31.1 \%$. Powder X-ray diffraction (Figure S7) confirmed that the single crystal used for structure determination was representative of the bulk product. IR $\left(\mathrm{cm}^{-1}\right)$ : $444.3(\mathrm{w}, \mathrm{b}), 509.9(\mathrm{~m}$, b), 570.3 (w, b), 614.0 (s), 636.0 (w, b), 724.4 (s), 734.9 (m), 742.4 (w), 748.0 (w), 770.5 (vs), 781.8 (s, sh), 827.9 (m), 833.6 (m), 895.4 (m, b), 955.4 (s), 992.9 (s), 1005.0 (w), 1019.1 (w), 1030.8 (w), 1047.2 (w, sh), 1065.0 (w), 1089.4 (w, sh), 1096.5 (m), 1108.2 (w), $1126.9(w), 1138.6$ (w, b), 1162.1 (m), 1176.1 (m), 1192.5 (m), 1216.0 (w, sh), 1232.4 (m), 1258.2 9m), 1269.9 (m, sh), 1284.0 (s), 1295.7 (s), 1328.5 (w), 1347.2 (w, sh), 1363.6 (w), 1370.7 (w, sh), 1410.5 (m, b), 1445.7 (s), 1464.4 (m), 1471.5 (w, sh), 1527.7 (vs), 1584.0 (vs), 1607.4 (vs), 2561.9 (w, b), 2721.2 (s, b, sh), 2787.6 (s, b), 2881.3 (s), 2948.9 (m, sh), 2984.6 (m, sh), 3054.9 (s), 3087.7 (s), 3139.3 (s, b), 3312.7 (s, vb), 3504.9 (w, b). Raman ( $\left.\mathrm{cm}^{-1}\right)$ : 164.3 (w), $185.2(\mathrm{w}), 226.6$ (m), $256.8(\mathrm{~m})$, $299.4(\mathrm{~m}), 329.4(\mathrm{w}), 347.7(\mathrm{w}), 368.8(\mathrm{w}), 402.3(\mathrm{w}), 532.6(\mathrm{w}, \mathrm{b}), 614.5(\mathrm{w}), 620.8(\mathrm{w}), 641.1$ (w), 717.7 (s), 723.9 (w, sh), 745.8 (w), 780.2 (w), 794.2 (w), 830.2 (m), 842.7 (w, sh), 995.8 (m), 1005.2 (vs), 1027.0 (m, sh), 1036.4 (s), 1064.5 (w), 1094.2 (w), 1165.1 (w), 1207.3 (w), 1229.8 (w), 1255.1 (s), 1277.6 (w, sh), 1297.3 (w, sh), 1311.4 (m, sh), 1328.2 (vs), 1345.1 (m, sh), 1367.6 (m, sh), 1412.6 (w), 1446.4 (m), 1466.1 (m), 1525.1 (m), 1572.9 (vs), 1584.2 (s), 1598.2 (vs, sh), 
1606.7 (vs), 1620.7 (vs, sh), 1637.6 (w, sh), 1651.7 (w, sh), 2851.0 (vs), 2873.1 (vs), 2912.5 (vs, b).

\section{Synthesis of Thorium(IV) Tetraaquo Tetrachloro Bis(4-chloropyridinium) Dichloride,}

$\left[\mathbf{T h}\left(\mathbf{H}_{2} \mathbf{O}\right)_{4} \mathbf{C l}_{4}\right] \cdot 2(4-\mathbf{C I P y H} \cdot \mathbf{C l}) ; 5$. $\mathrm{ThCl}_{4}(0.049 \mathrm{~g}, 0.132 \mathrm{mmol})$ was dissolved in hydrochloric $\operatorname{acid}\left(\mathrm{HCl}_{(a q)}, 500 \mu \mathrm{L}, 3 \mathrm{M}\right)$ to generate an acidic aqueous solution of $\mathrm{Th}^{\mathrm{IV}}(0.262 \mathrm{M})$. The vial was subsequently charged with 4-chloropyridine hydrochloride $(19.8 \mathrm{mg}, 0.132 \mathrm{mmol})$ to yield a colorless solution, which was left to evaporate under a nitrogen atmosphere. After approximately 7 days, colorless block crystals formed at the bottom of the vial. Single crystal X-ray diffraction determined the crystals to be $\left[\mathrm{Th}\left(\mathrm{H}_{2} \mathrm{O}\right)_{4} \mathrm{Cl}_{4}\right] \cdot 2(4-\mathrm{ClPyH} \cdot \mathrm{Cl})$ (5). The bulk phase, however, dissolves readily under ambient conditions. Due to the sensitivity of this compound, further analyses to assess both the yield and phase purity of this reaction could not be feasibly performed. Therefore, yield determination, powder X-ray diffraction, and elemental analysis are not reported. IR ( $\left.\mathrm{cm}^{-1}\right)$ : 422.6 (s, b), 488.3 (vs, b), 570.8 (s, b), 724.5 (m), 786.4 (s), 812.6 (s), 915.3 (w), 945.3 (w), $990.3(w), 996.0(w), 1011.9(w), 1019.7$ (w, sh), $1057.2(w), 1085.3(\mathrm{~m}), 111.9(\mathrm{~s}), 1152.6$ (w, b), 1190.1 (m), 1199.5 (m, sh), 1240.1 (m, sh), 1244.8 (m), 1278.6 (w, b), 1303.6 (m), 1330.2 (w), 1345.8 (m), 1359.9 (w), 1367.7 (w), 1476.1 (vs), 1479.2 (vs, sh), 1598.2 (vs), 1621.6 (vs), 1654.2 (w), 2892.2 (m, sh), 2931.6 (m, sh), 2955.7 (m, sh), 3049.7 (vs), 3067.2 (vs, sh), 3080.4 (vs, sh), 3089.1 (vs, sh), 3124.1 (vs, b), 3199.7 (s), 3269.8 (m, b). Raman ( $\left.\mathrm{cm}^{-1}\right)$ : 158.9 (w), 176.6 (w), 207.8 (w), 237.5 (w), 279.7 (w, sh), 306.3 (w) $426.6(\mathrm{~m}), 485.9$ (w), $643.1(\mathrm{~m}), 725.3(\mathrm{~m})$, 1008.5 (s, sh), 1013.8 (vs), 1058.4 (m), 1109.7 (s), 1192.8 (w), 1200.6 (w, sh), 1230.3 (w), 1242.8 (w), 1444.4 (w, b), 1599.9 (w), 1621.2 (m), 2890.5 (s, vb), 3058.4 (s, sh), 3069.0 (s, sh), 3085.9 (vs), $3150.0(\mathrm{w}, \mathrm{b})$. 


\section{CRYSTALLOGRAPHIC REFINEMENT DETAILS OF COMPOUNDS 1-5.}

Note: Crystallographic refinement details can be found in the "experimental refinement section" of the crystallographic information files (CIFs). Details are also provided below for ease of the reader.

For all compounds, non-hydrogen atoms were located in the Fourier difference map and refined anisotropically. Hydrogen atoms bound to carbons of heterocyclic rings were placed in calculated positions and their $\mathrm{U}_{e q}$ values were assigned values 1.2 times that of their parent atom. If present, hydrogen atoms bound to heteroatoms (nitrogen and oxygen) were found in the Fourier difference map unless otherwise stated below. If the $\mathrm{X}-\mathrm{H}$ distance $(\mathrm{X}=\mathrm{N}, \mathrm{O})$ was not within reasonable limits, the distance was fixed; for water molecules, $\mathrm{O}-\mathrm{H}$ distances were fixed at 0.88(0.02) $\AA$. For the protonated nitrogen atoms contained within heterocyclic counterions, $\mathrm{N}-\mathrm{H}$ distances were fixed to $0.86(0.02) \AA$. Protonated nitrogen and $\mathrm{H}$ atom $\mathrm{U}_{e q}$ values were assigned as 1.5 times their carrier atom.

Compound 1: C3 was restrained to behave relatively isotropic. One hydroxyl H atom was located in the difference map and was restrained to a distance of $0.97(0.02) \AA$ with the $\mathrm{H}$ atom $\mathrm{U}_{e q}$ assigned as 1.5 times the parent atom. The distance between the Th and hydrogen bound to $\mathrm{O} 2$ was set in a fixed position. The angle between the $\mathrm{H}$ atoms bound to $\mathrm{O} 4$ was also set to a fixed angle.

Compound 2: Some of the outer coordination sphere molecules were disordered. As such, the lattice water molecule was disordered over two positions and one of the 2-methylpyridinium cations was disordered over two positions; both parts were restrained to possess similar anisotropic $\mathrm{U}$ values. The distance between the pyridinium ring and $2-\mathrm{CH}_{3}$ group was restrained to a similar distance and two atoms of the 2-methylpyridinium were restrained to behave relatively isotropic. The angle between the hydrogen atoms bound to $\mathrm{O} 1$ and $\mathrm{O} 3$ were also set to a fixed angle. The $\mathrm{N}(2)-\mathrm{H}$ hydrogen atom could not be located in the difference map and therefore was placed in a

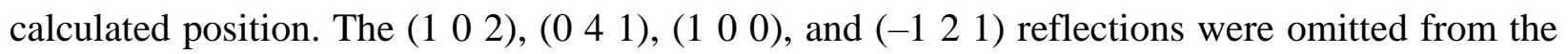
final refinement due to obstruction from the beam stop. 
Compound 3: Some of the outer coordination sphere molecules were disordered. As such, the lattice chloride ion was disordered over two positions and one of the 4-chloropyridinium cations was disordered across a symmetry site over two orientations. The 4-chloropyridinium rings were constrained to be ideal hexagons. The like $\mathrm{Cl}-\mathrm{C}$ distances were restrained to be similar (esd 0.01 $\AA$ ). The $\mathrm{C} 4$ and $\mathrm{Cl} 5 \mathrm{~B}$ atoms were constrained to have equal anisotropic displacement parameters. Similar displacement amplitudes (esd 0.01) were imposed on disordered sites overlapping by less than the sum of van der Waals radii. The 4-chloropyridinium $\mathrm{H}$ atoms bound to the nitrogens of the disordered cation could not be located in the difference map and thus were place in calculated positions.

Compound 4: The structure was refined as a 2-component inversion twin. One of the lattice water molecules was disordered over two positions and restrained to behave isotropically. Additionally, the hydrogens of this lattice water molecule could not be located in the difference map and therefore were left out of the model. The angle between the hydrogen atoms bound to O5 was fixed.

Compound 5: $\mathrm{O} 2$ was restrained to behave relatively isotropic and possess rigid bond restraints. The distance between the Th and HO2B was set in a fixed position. The angle between $\mathrm{H}$ atoms bound to $\mathrm{O} 2$ was also set to a fixed angle. 


\section{ORTEP DIAGRAMS OF COMPOUNDS 1-5.}

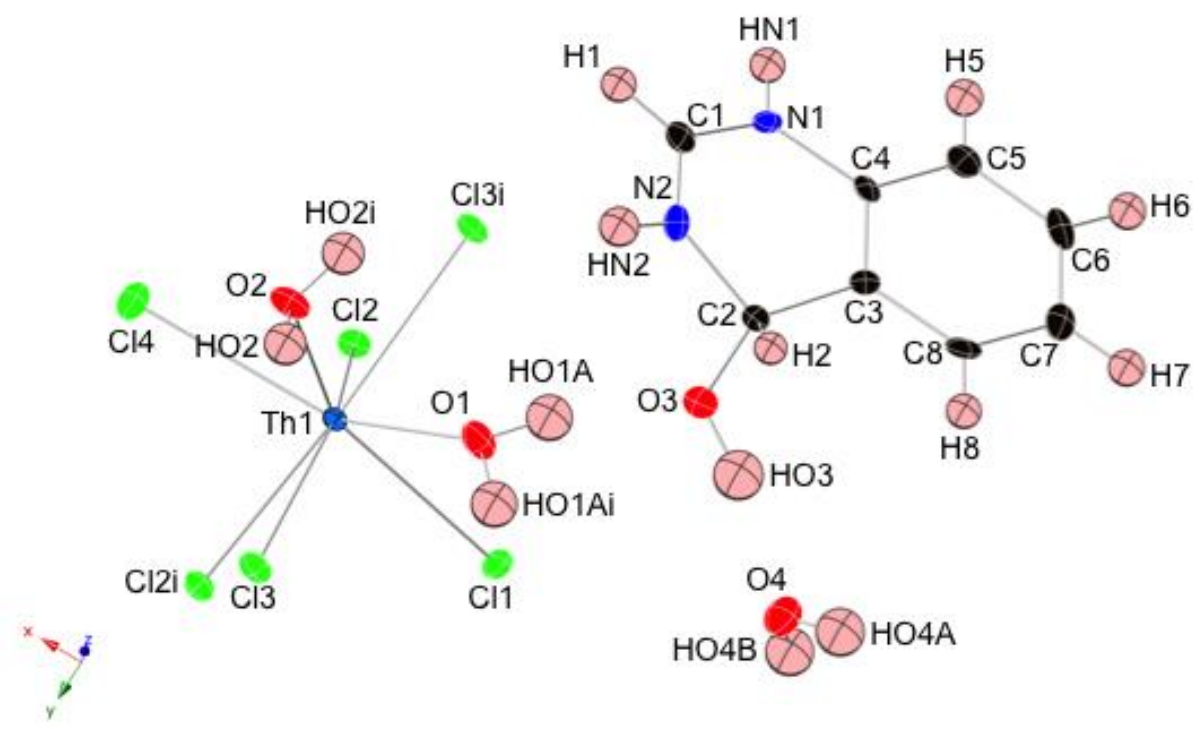

Figure S1. Thermal ellipsoid plot $(50 \%$ probability level $)$ of compound 1, (QuinoH $)_{2}\left[\mathrm{Th}\left(\mathrm{H}_{2} \mathrm{O}\right)_{2} \mathrm{Cl}_{6}\right] \cdot \mathrm{H}_{2} \mathrm{O}$, at $100 \mathrm{~K}$. Symmetry equivalent atoms were generated through their respective symmetry elements $(i=x,-y+3 / 2, z)$.

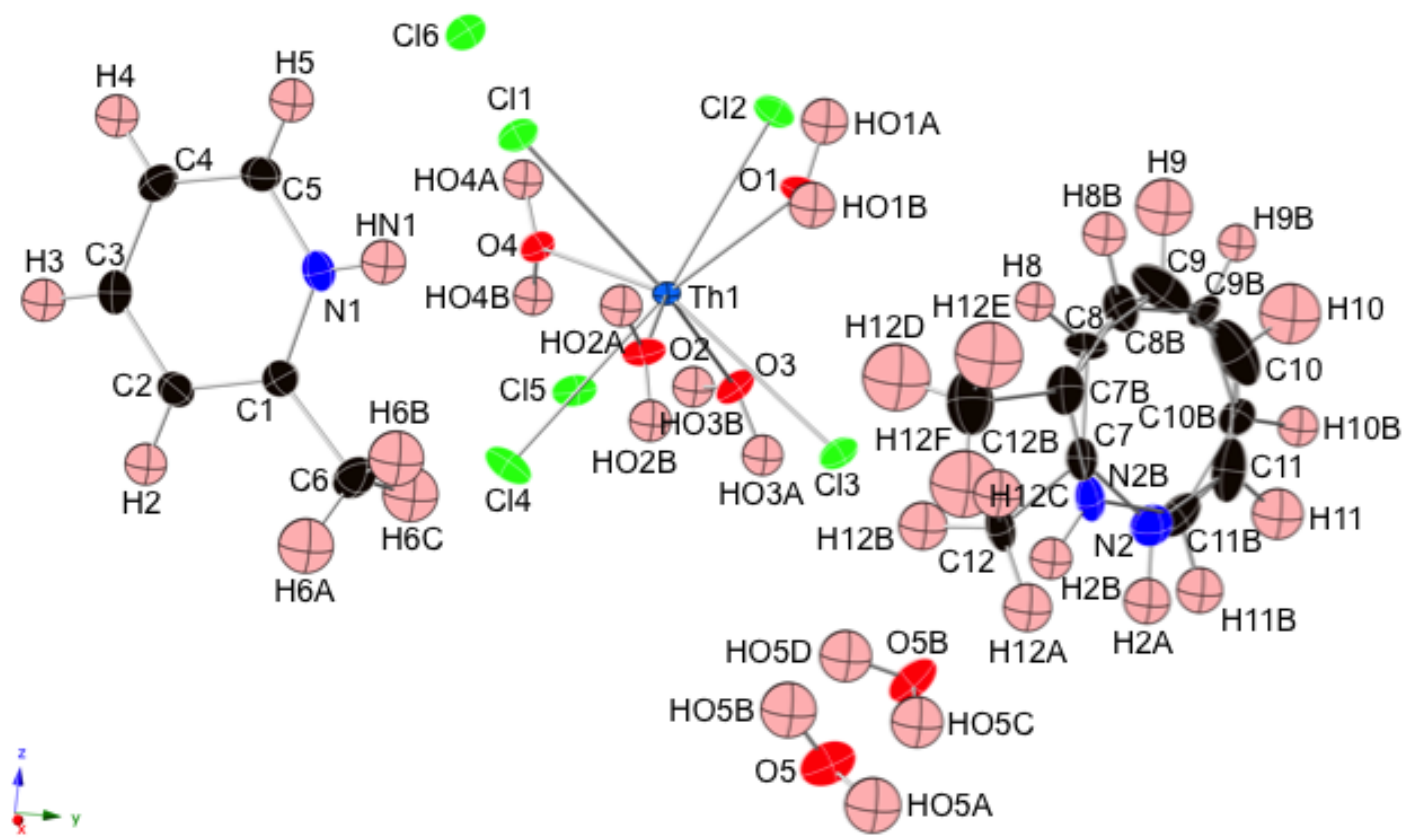

Figure S2. Thermal ellipsoid plot (50\% probability level) of compound 2, $\left[\mathrm{Th}\left(\mathrm{H}_{2} \mathrm{O}\right)_{4} \mathrm{Cl}_{4}\right] \cdot 2(2-$ $\mathrm{MePyH} \cdot \mathrm{Cl}) \cdot \mathrm{H}_{2} \mathrm{O}$, at $100 \mathrm{~K}$. The disorder in the structure has been included in the plot. 


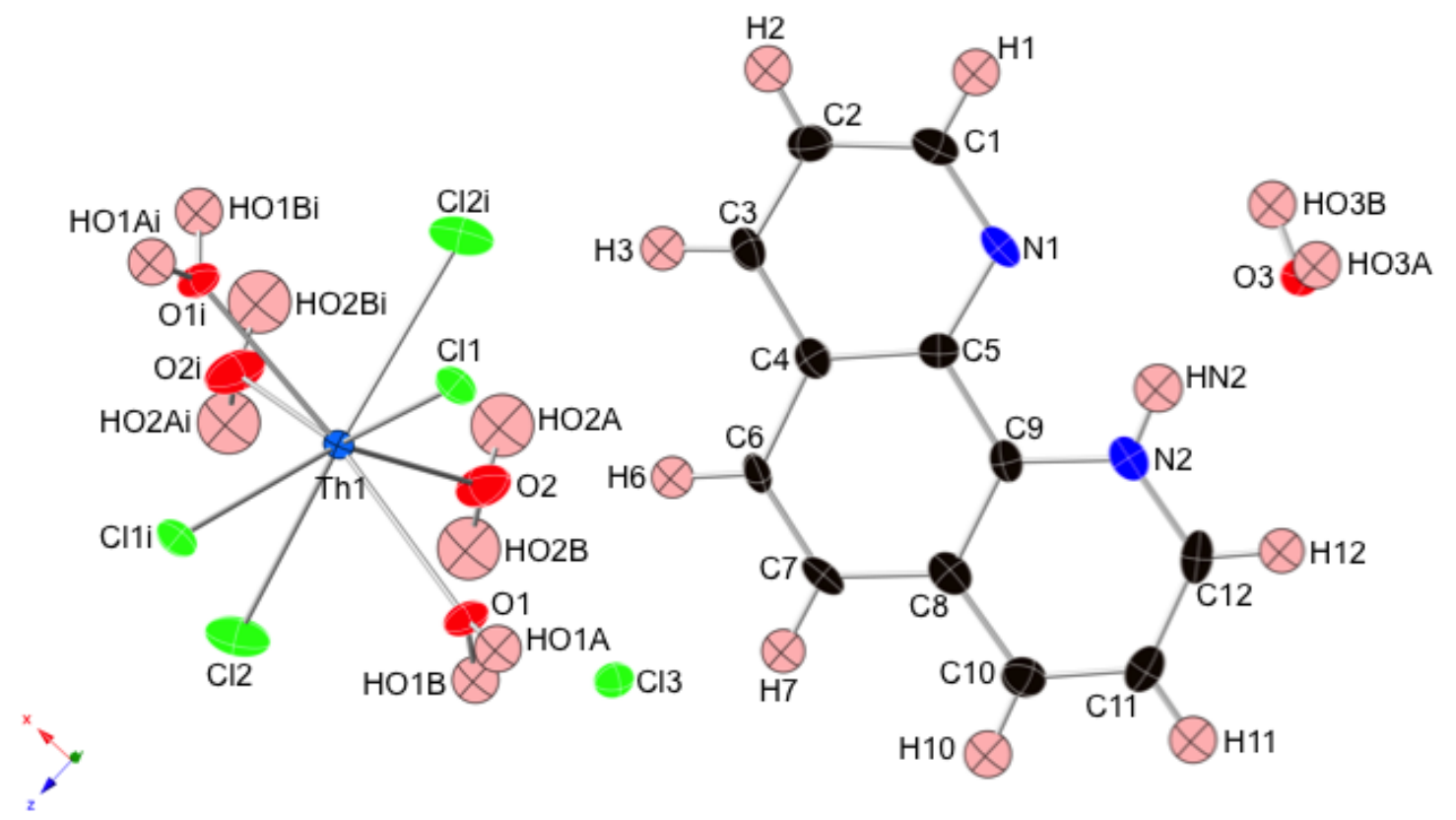

Figure S3. Thermal ellipsoid plot $(50 \%$ probability level) of compound 3, $\left[\mathrm{Th}\left(\mathrm{H}_{2} \mathrm{O}\right)_{4} \mathrm{Cl}_{4}\right] \cdot 2(\mathrm{PhenH} \cdot \mathrm{Cl}) \cdot 2 \mathrm{H}_{2} \mathrm{O}$, at $100 \mathrm{~K}$. Symmetry equivalent atoms were generated through their respective symmetry elements $(\mathrm{i}=-\mathrm{x}+1, \mathrm{y},-\mathrm{z}+3 / 2)$.

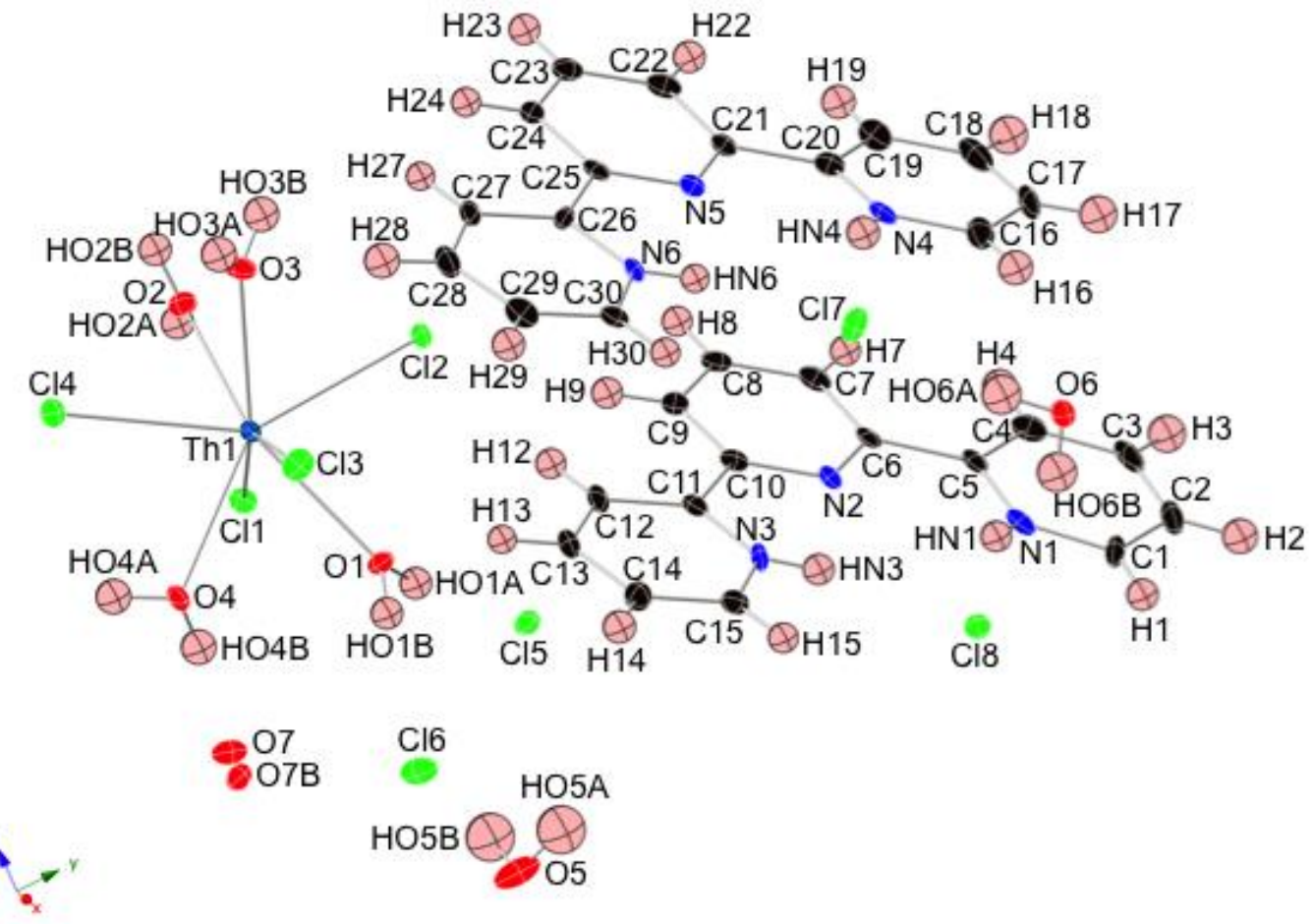

Figure S4. Thermal ellipsoid plot $(50 \%$ probability level $)$ of compound 4, $\left[\mathrm{Th}\left(\mathrm{H}_{2} \mathrm{O}\right)_{4} \mathrm{Cl}_{4}\right] \cdot 2\left(\mathrm{TerpyH}_{2} \cdot 2 \mathrm{Cl}\right) \cdot 3 \mathrm{H}_{2} \mathrm{O}$, at $100 \mathrm{~K}$. The minor disorder in the structure has been included in the plot. 


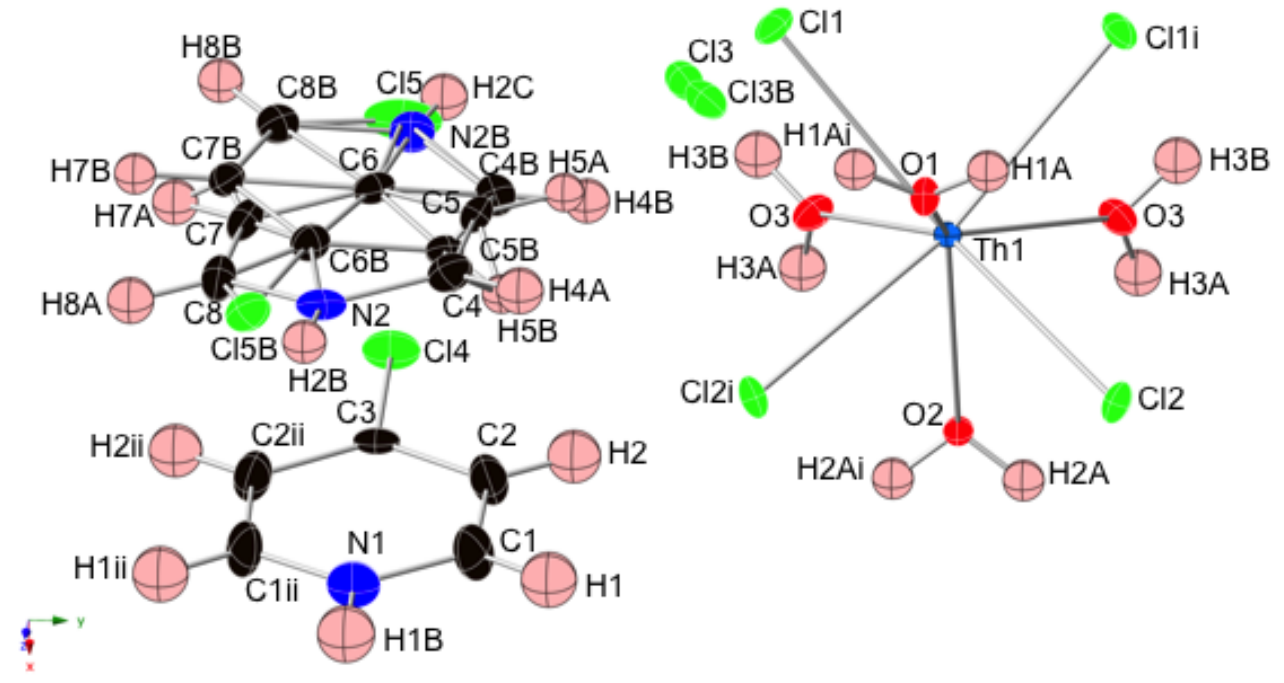

Figure S5. Thermal ellipsoid plot (50\% probability level) of compound 5, [Th( $\left.\left.\mathrm{H}_{2} \mathrm{O}\right)_{4} \mathrm{Cl}_{4}\right] \cdot 2(4-$ $\mathrm{ClPyH} \cdot \mathrm{Cl})$, at $100 \mathrm{~K}$. Symmetry equivalent atoms were generated through their respective symmetry elements $(\mathrm{i}=\mathrm{x},-\mathrm{y}+3 / 2, \mathrm{z} ; \mathrm{ii}=\mathrm{x},-\mathrm{y}+1 / 2, \mathrm{z})$. The disorder in the structure has been included in the plot. 


\section{POWDER X-RAY DIFFRACTION PLOTS OF COMPOUNDS 3-4.}

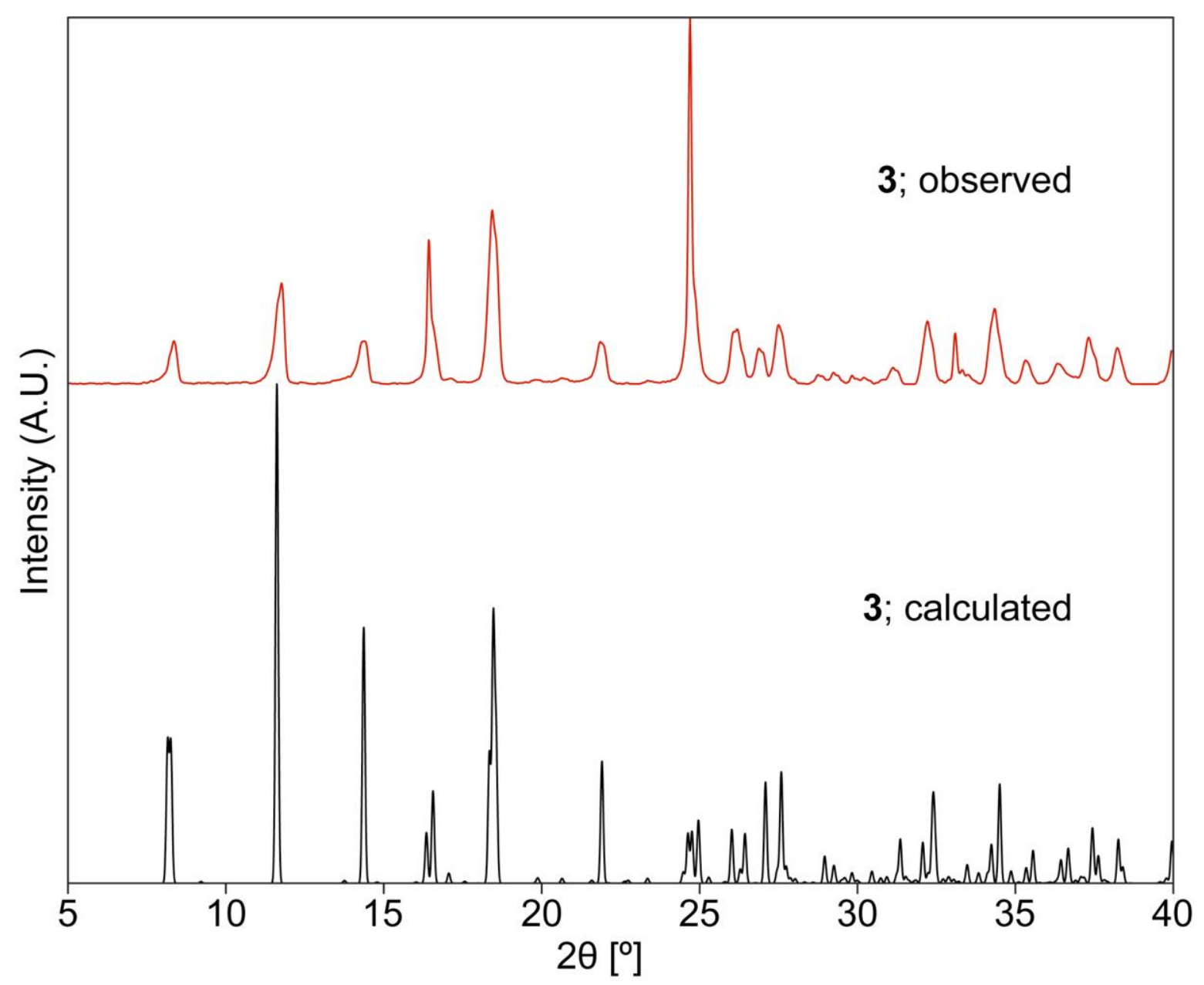

Figure S6. Powder X-ray diffraction pattern (collected with $\mathrm{Cu}$ Ka radiation) observed for 3 (red) overlaid with the pattern calculated from the single crystal structure of $\mathbf{3}$ at $100 \mathrm{~K}$ (black). 


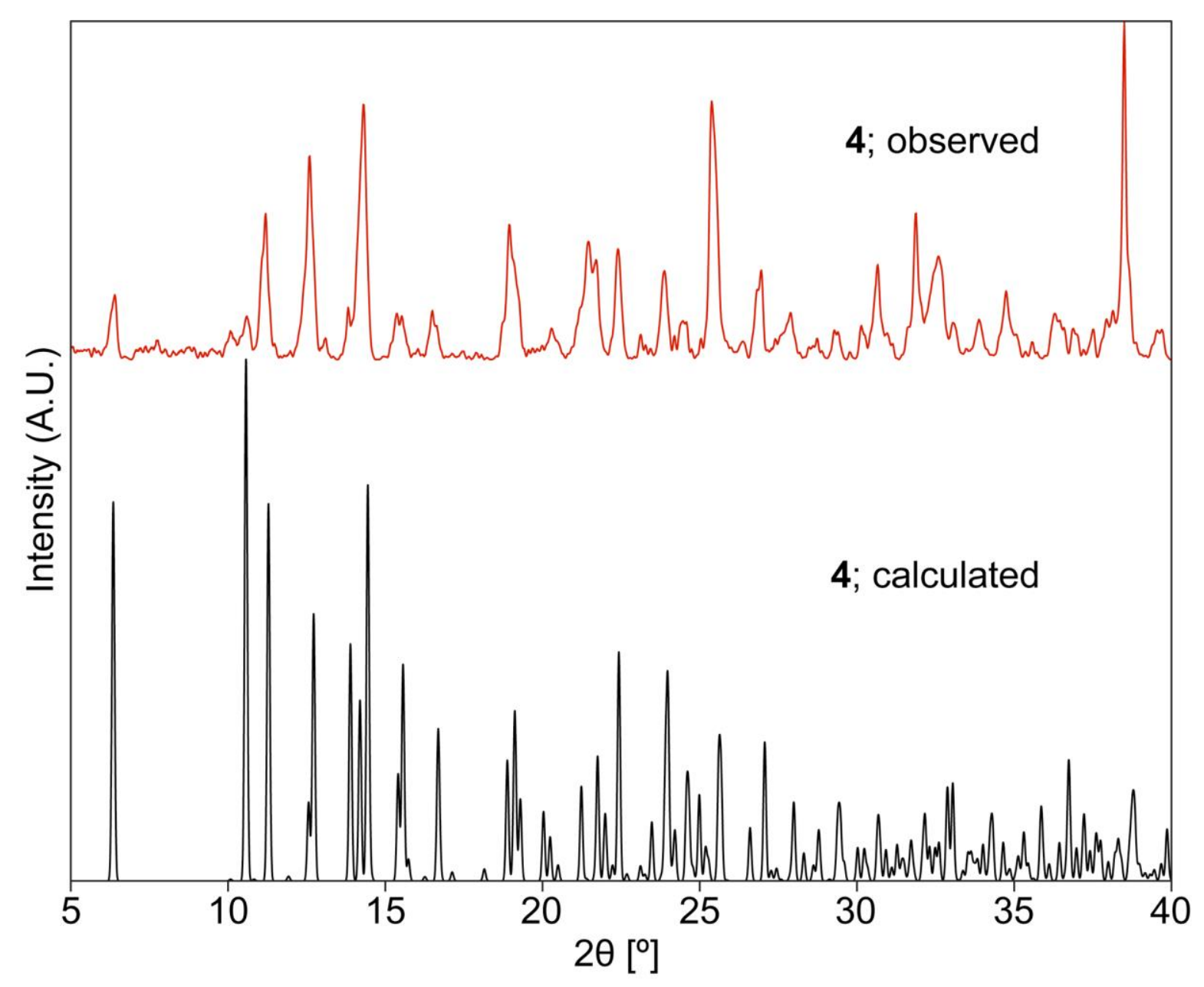

Figure S7. Powder X-ray diffraction pattern (collected with $\mathrm{Cu}$ Ka radiation) observed for $\mathbf{4}$ (red) overlaid with the pattern calculated from the single crystal structure of $\mathbf{4}$ at $100 \mathrm{~K}$ (black). 


\section{RAMAN AND INFRARED PLOTS OF COMPOUNDS 1-5.}

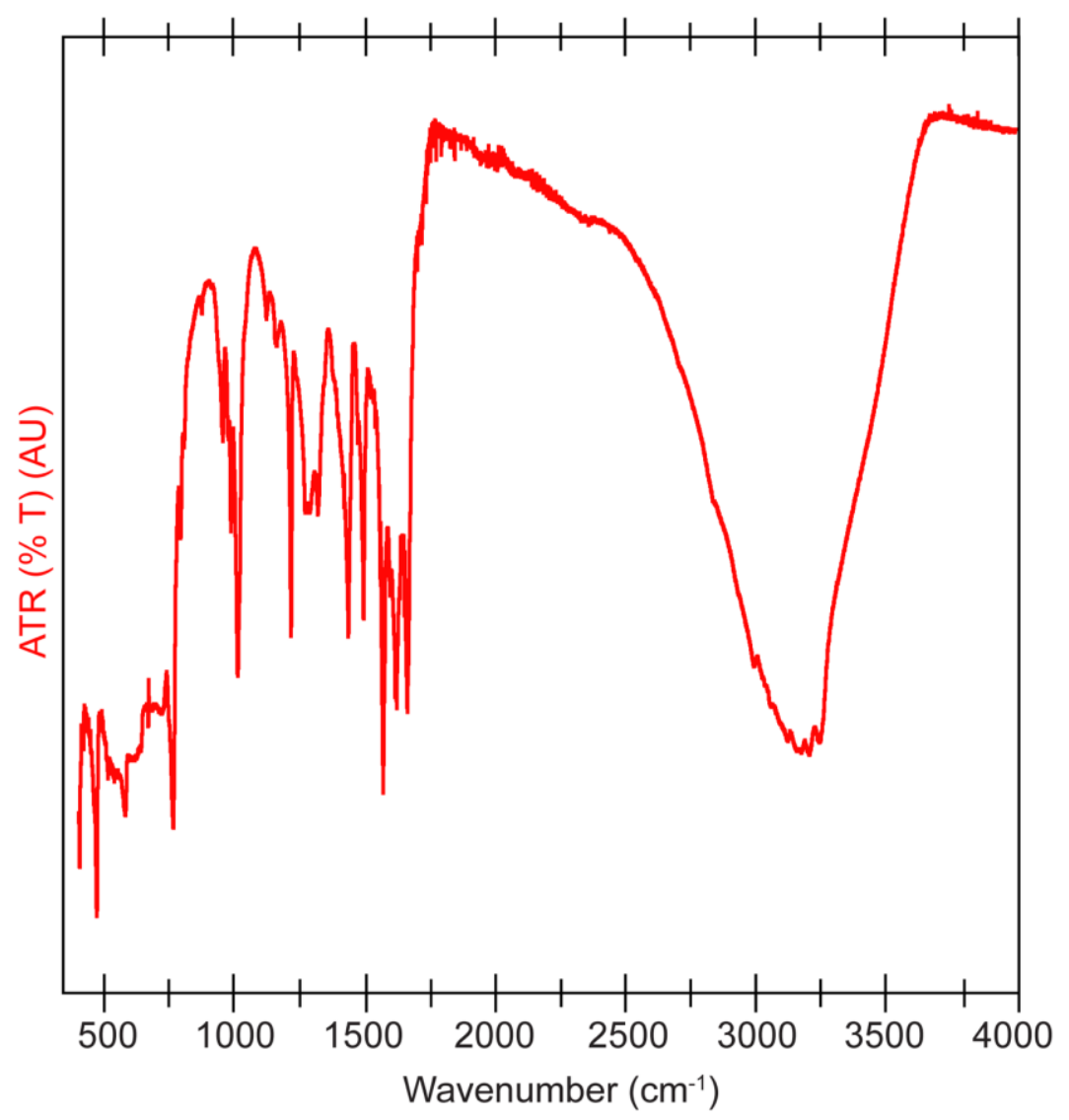

Figure S8. Infrared spectrum of compound 1, (QuinoH $)_{2}\left[\mathrm{Th}\left(\mathrm{H}_{2} \mathrm{O}\right)_{2} \mathrm{Cl}_{6}\right] \cdot \mathrm{H}_{2} \mathrm{O}$.

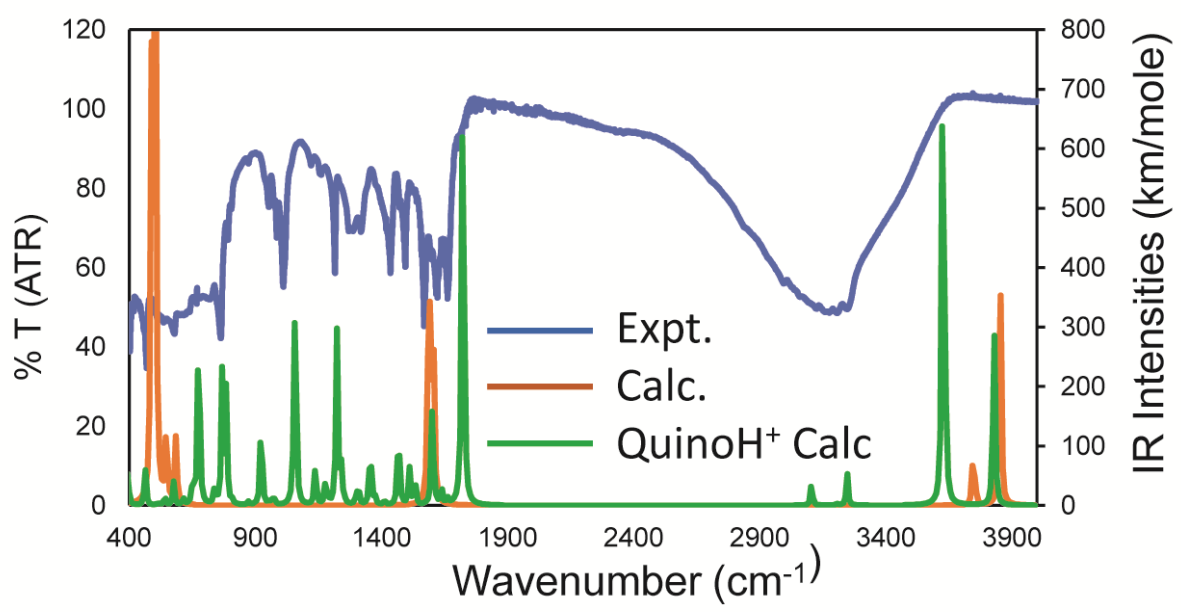

Figure S9. Comparison of calculated infrared frequencies (orange and green) versus experimental observations for compound 1 (blue) shown over $400-4000 \mathrm{~cm}^{-1}$. 
(a)

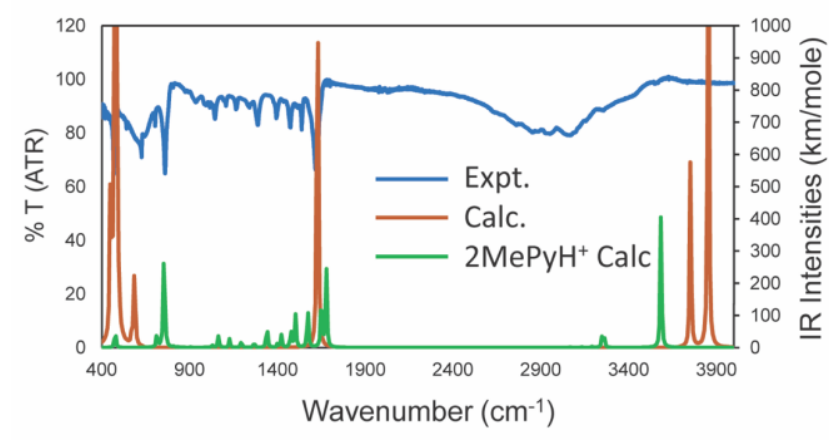

(b)

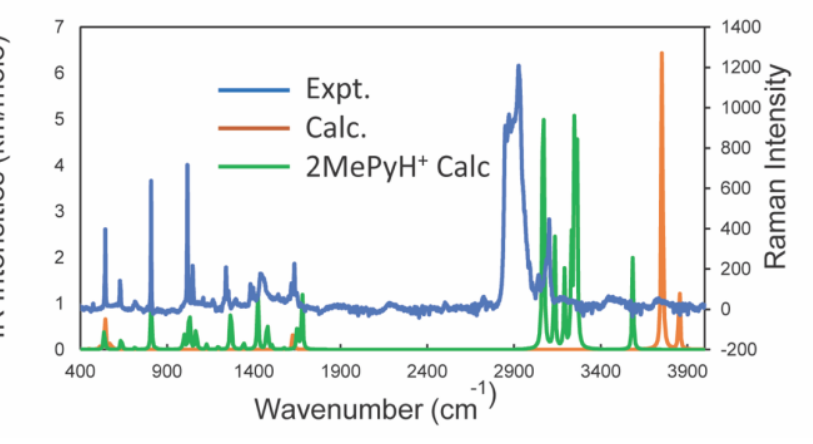

Figure S10. Comparison of (a) calculated infrared frequencies (orange and green) versus experimental observations and (b) calculated Raman frequencies (orange and green) versus experimental observations (blue) for compound 2 shown over $400-4000 \mathrm{~cm}^{-1}$.

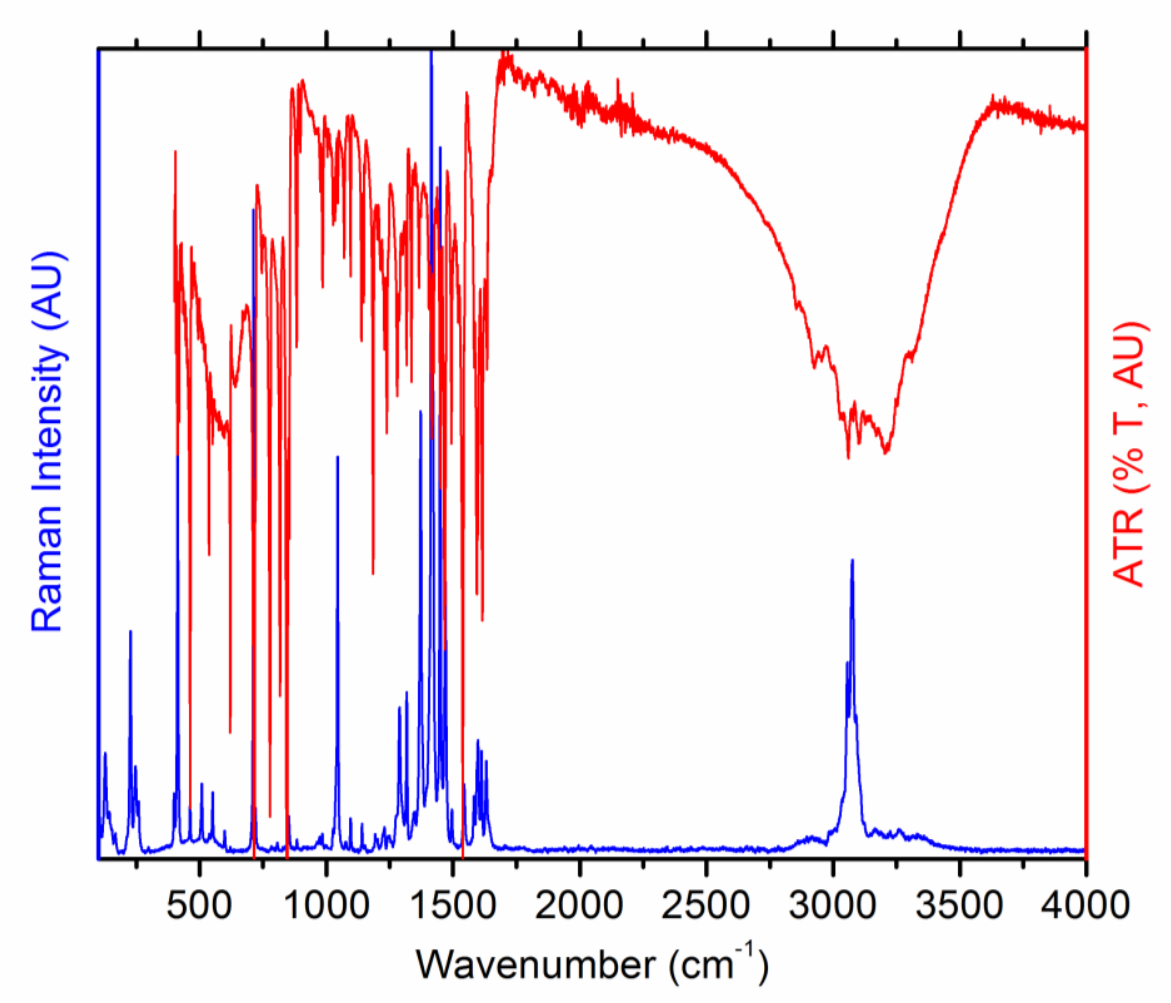

Figure S11. Raman and infrared spectra of compound 3, $\left[\mathrm{Th}\left(\mathrm{H}_{2} \mathrm{O}\right)_{4} \mathrm{Cl}_{4}\right] \cdot 2(\mathrm{PhenH} \cdot \mathrm{Cl}) \cdot 2 \mathrm{H}_{2} \mathrm{O}$ (Raman = blue, IR = red). 
(a)

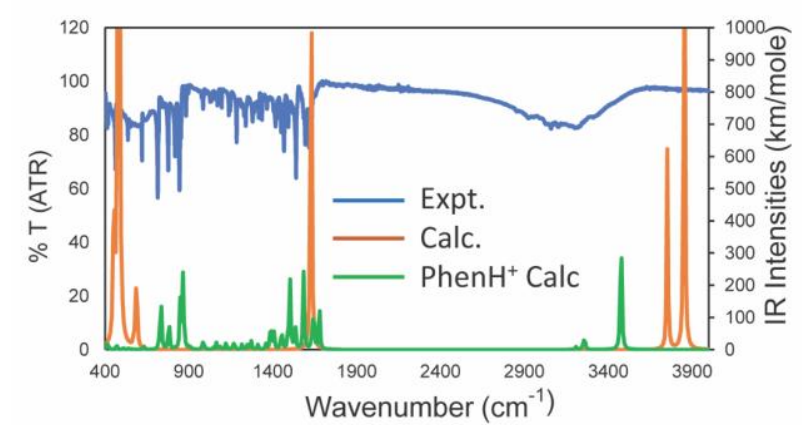

(b)

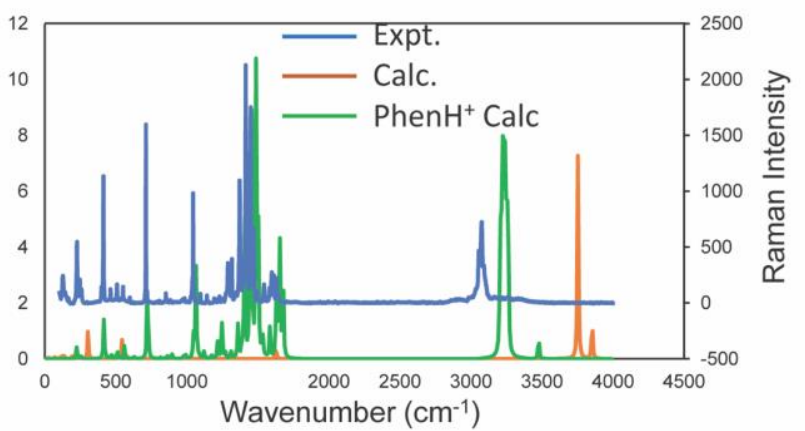

Figure S12. Comparison of (a) calculated infrared frequencies (orange and green) versus experimental observations and (b) calculated Raman frequencies (orange and green) versus experimental observations (blue) for compound 3 shown over $400-4000 \mathrm{~cm}^{-1}$.

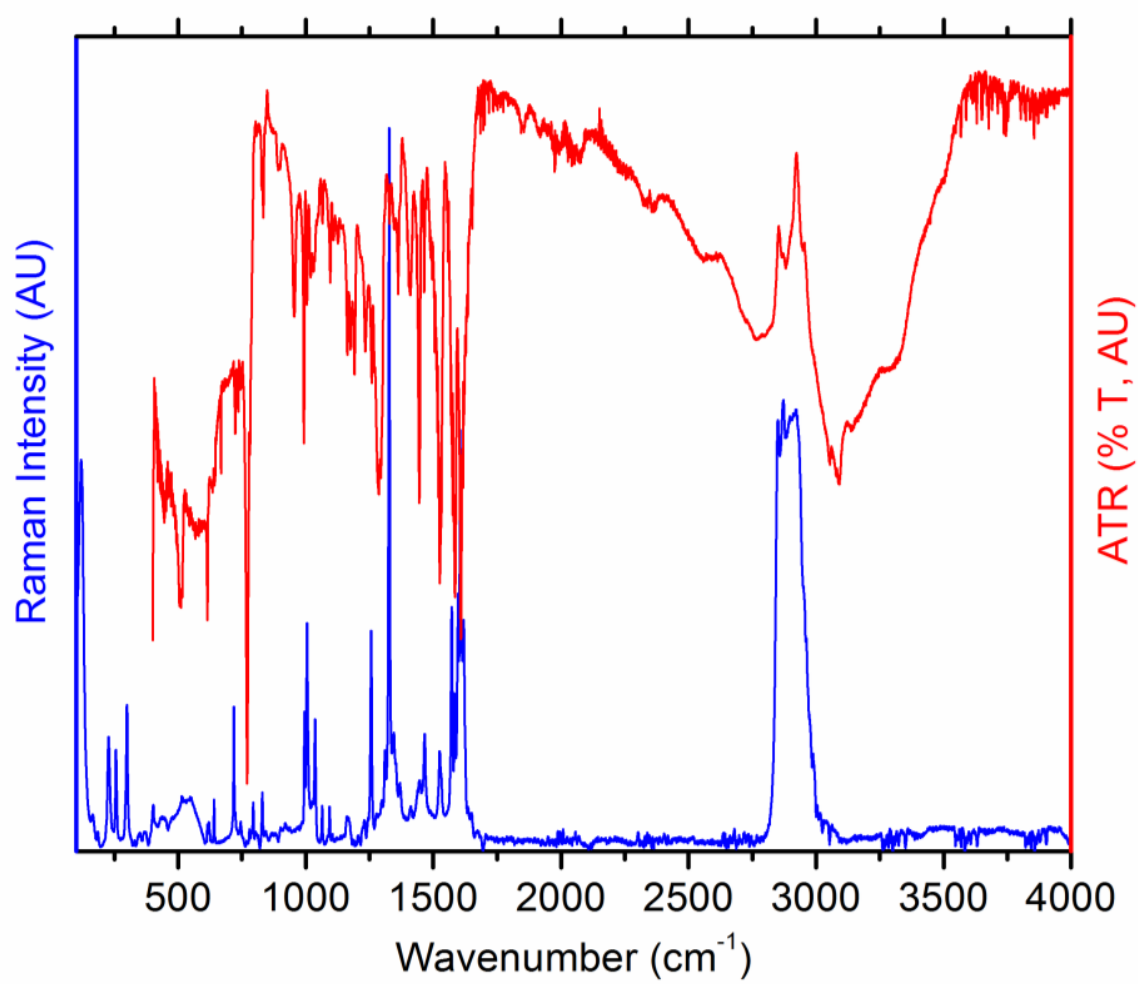

Figure S13. Raman and infrared spectra of compound 4, $\left[\mathrm{Th}\left(\mathrm{H}_{2} \mathrm{O}\right)_{4} \mathrm{Cl}_{4}\right] \cdot 2\left(\mathrm{TerpyH} \mathrm{H}_{2} \cdot 2 \mathrm{Cl}\right) \cdot 3 \mathrm{H}_{2} \mathrm{O}$ (Raman = blue, IR = red). 
(a)

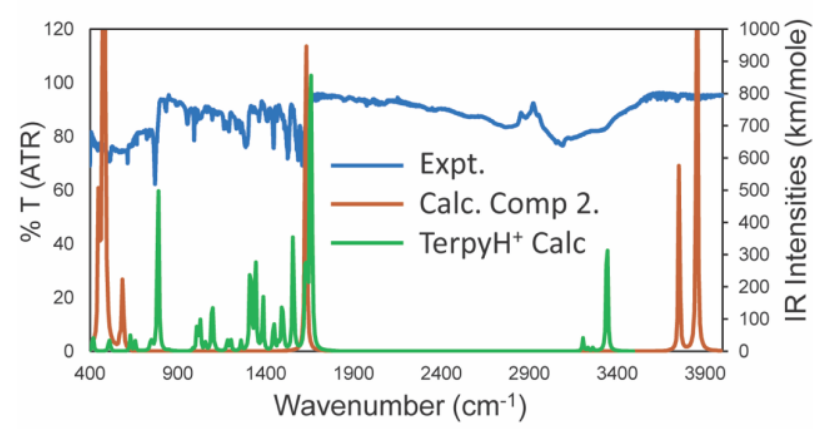

(b)

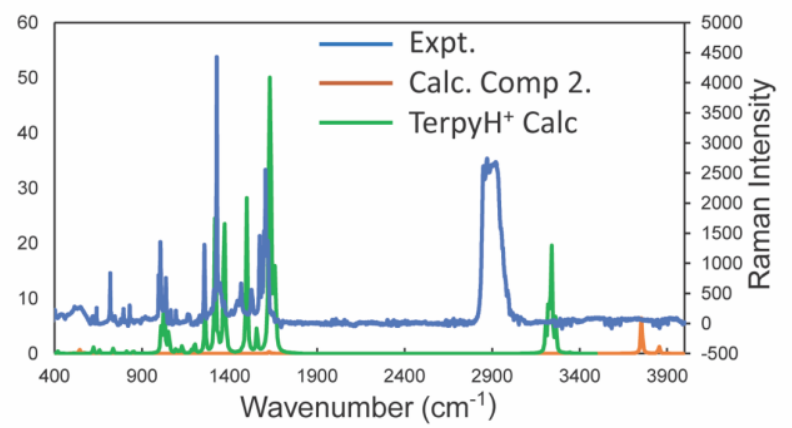

Figure S14. Comparison of (a) calculated infrared frequencies (orange and green) versus experimental observations and (b) calculated Raman frequencies (orange and green) versus experimental observations (blue) for compound 4 shown over $400-4000 \mathrm{~cm}^{-1}$.

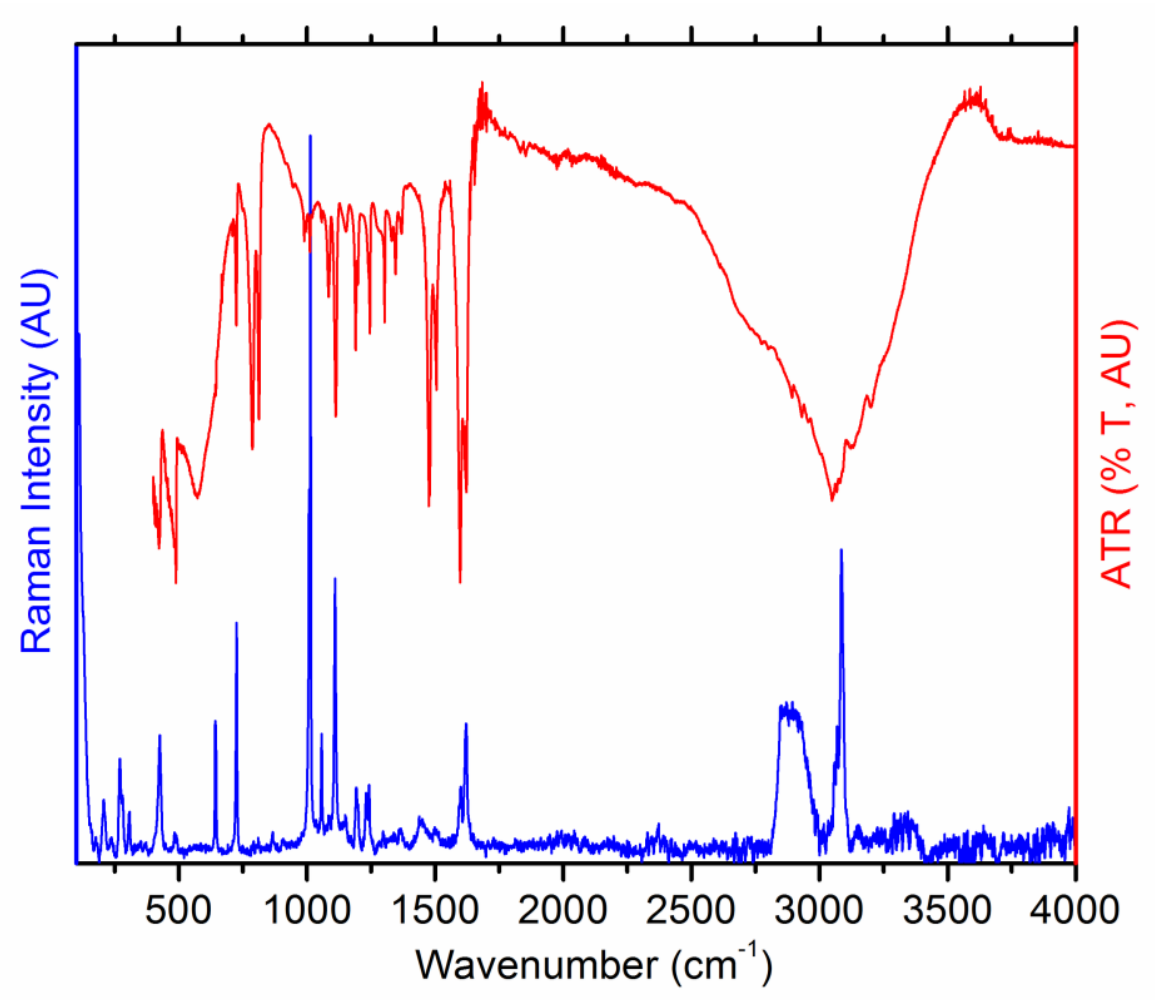

Figure S15. Raman and infrared spectra of compound 5, $\left[\mathrm{Th}\left(\mathrm{H}_{2} \mathrm{O}\right)_{4} \mathrm{Cl}_{4}\right] \cdot 2(4-\mathrm{ClPyH} \cdot \mathrm{Cl})(\mathrm{Raman}=$ blue, IR = red). 
(a)

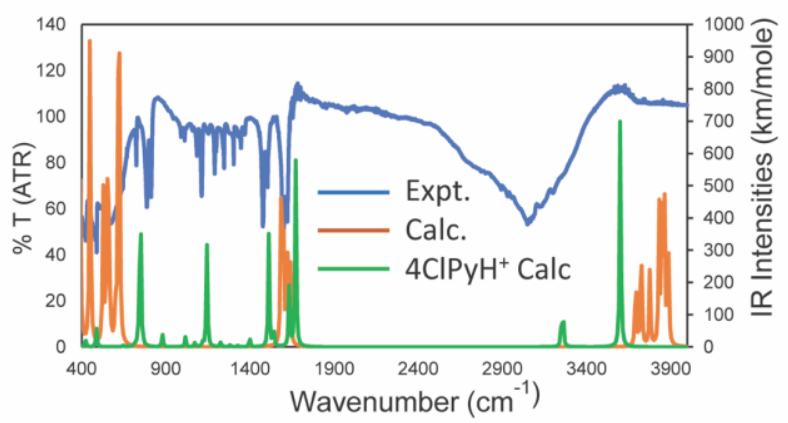

(b)

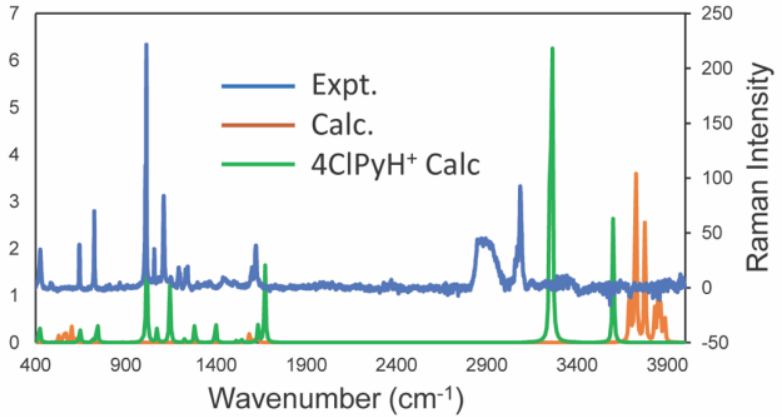

Figure S16. Comparison of (a) calculated infrared frequencies (orange and green) versus experimental observations and (b) calculated Raman frequencies (orange and green) versus experimental observations (blue) for compound 5 shown over $400-4000 \mathrm{~cm}^{-1}$. 


\section{SUPRAMOLECULAR INTERACTIONS OBSERVED IN COMPOUNDS 1-5.}

Supramolecular interactions were calculated using the "Calc All" function within the PLATON software suite. ${ }^{1}$ Significant noncovalent interactions for reported compounds - such as hydrogen bonding, halogen $-\pi, \mathrm{C}-\mathrm{H}-\pi$, and $\pi-\pi$ stacking interactions - are tabulated below, guided by the classification of supramolecular interactions as reported previously. ${ }^{2-4}$

\section{Noncovalent Interaction Notations:}

- $\mathrm{D}=$ donor atom involved in supramolecular interaction.

- $\mathrm{A}=$ acceptor atom involved in supramolecular interaction.

- $\beta=$ angle formed between $\mathrm{C}_{\mathrm{g}}$ and the plane normal to $\mathrm{C}_{\mathrm{g}}$.

- $\gamma=$ angle formed between the ring normal of the plane and centroid vector.

- $\mathrm{C}_{\mathrm{g}}---\mathrm{C}_{\mathrm{g}}=$ Distance between ring centroids.

Table S1. Selected noncovalent interactions - such as hydrogen bonding, halogen $-\pi, \mathrm{C}-\mathrm{H}-\pi$, and $\pi-\pi$ stacking interactions - observed in $(\text { QuinoH })_{2}\left[\mathrm{Th}\left(\mathrm{H}_{2} \mathrm{O}\right)_{2} \mathrm{Cl}_{6}\right] \cdot \mathrm{H}_{2} \mathrm{O}(\mathbf{1}) \cdot \mathrm{Cg}_{\mathrm{g} 2}=$ centroid formed from ring containing atoms $\mathrm{C}_{3}, \mathrm{C}_{4}, \mathrm{C}_{5}, \mathrm{C}_{6}, \mathrm{C}_{7}, \mathrm{C}_{8}$.

\begin{tabular}{|c|c|c|c|c|}
\hline Interaction & $\begin{array}{c}\text { Distance }(\AA), \\
\text { D-H---A }\end{array}$ & $\begin{array}{l}\text { Angle }\left(^{\circ}\right), \\
\angle \mathrm{D}-\mathrm{H}---\mathrm{A}\end{array}$ & $\begin{array}{c}\text { Distance }(\AA), \\
\mathrm{C}_{\mathrm{g}}--\mathrm{C}_{\mathrm{g}}\end{array}$ & $\beta\left({ }^{\circ}\right)$ \\
\hline $\mathrm{O}(1)-\mathrm{H}--\mathrm{O}(3)$ & $2.645(6)$ & 161(4) & - & - \\
\hline $\begin{array}{c}\text { intra } \\
\mathrm{O}(2)-\mathrm{H}---\mathrm{Cl}(3)\end{array}$ & $3.126(3)$ & $103.1(17)$ & - & - \\
\hline $\mathrm{O}(2)-\mathrm{H}---\mathrm{Cl}(2)$ & $3.145(4)$ & $152(2)$ & - & - \\
\hline $\mathrm{O}(3)-\mathrm{H}$--- $\mathrm{O}(4)$ & $2.691(6)$ & $170(4)$ & - & - \\
\hline $\mathrm{O}(4)-\mathrm{H}---\mathrm{O}(2)$ & $2.896(8)$ & 177(4) & - & - \\
\hline $\mathrm{O}(4)-\mathrm{H}$--- $\mathrm{Cl}(4)$ & $3.243(6)$ & $178(5)$ & - & - \\
\hline $\mathrm{N}(1)-\mathrm{H}---\mathrm{Cl}(2)$ & $3.355(4)$ & $165(4)$ & - & - \\
\hline $\mathrm{N}(2)-\mathrm{H}$--- $\mathrm{Cl}(1)$ & $3.330(4)$ & $137(3)$ & - & - \\
\hline $\mathrm{N}(2)-\mathrm{H}$--- $\mathrm{Cl}(2)$ & $3.381(4)$ & 141(3) & - & - \\
\hline $\mathrm{C}_{\mathrm{g} 2---} \mathrm{C}_{\mathrm{g} 2}$ & - & - & $3.635(3)$ & 22.4 \\
\hline $\mathrm{C}_{\mathrm{g} 2}---\mathrm{C}_{\mathrm{g} 2}$ & - & - & $3.728(3)$ & 26.6 \\
\hline
\end{tabular}


Table S2. Selected noncovalent interactions - such as hydrogen bonding, halogen $-\pi, \mathrm{C}-\mathrm{H}-\pi$, and $\pi-\pi$ stacking interactions - observed in $\left[\mathrm{Th}\left(\mathrm{H}_{2} \mathrm{O}\right)_{4} \mathrm{Cl}_{4}\right] \cdot 2(2-\mathrm{MePyH} \cdot \mathrm{Cl}) \cdot \mathrm{H}_{2} \mathrm{O}(\mathbf{2}) \cdot \mathrm{C}_{\mathrm{g} 1}=$ centroid formed from ring containing atoms $\mathrm{N}_{1}, \mathrm{C}_{1}, \mathrm{C}_{2}, \mathrm{C}_{3}, \mathrm{C}_{4}, \mathrm{C}_{5}$.

\begin{tabular}{|c|c|c|c|c|}
\hline Interaction & $\begin{array}{c}\text { Distance }(\AA), \\
\text { D-H---A }\end{array}$ & $\begin{array}{l}\text { Angle }\left(^{\circ}\right), \\
\angle \mathrm{D}-\mathrm{H}---\mathrm{A}\end{array}$ & $\begin{array}{c}\text { Distance }(\AA), \\
\text { C-H---Cg }\end{array}$ & $\gamma\left({ }^{\circ}\right)$ \\
\hline $\mathrm{O}(1)-\mathrm{H}$--- $\mathrm{Cl}(3)$ & $3.127(2)$ & $162(2)$ & - & - \\
\hline $\mathrm{O}(1)-\mathrm{H}--\mathrm{O}(5)$ & $2.665(3)$ & 161(3) & - & - \\
\hline $\mathrm{N}(2)-\mathrm{H}---\mathrm{Cl}(6)$ & $3.347(6)$ & 160 & - & - \\
\hline $\mathrm{O}(2)-\mathrm{H}---\mathrm{Cl}(6)$ & $3.069(2)$ & $165(2)$ & - & - \\
\hline $\mathrm{O}(2)-\mathrm{H}---\mathrm{Cl}(5)$ & $3.103(2)$ & $165(2)$ & - & - \\
\hline $\mathrm{O}(3)-\mathrm{H}---\mathrm{Cl}(2)$ & $3.219(2)$ & $159(2)$ & - & - \\
\hline $\mathrm{O}(3)-\mathrm{H}--\mathrm{Cl}(5)$ & $3.043(2)$ & $175(2)$ & - & - \\
\hline $\mathrm{O}(4)-\mathrm{H}---\mathrm{Cl}(6)$ & $3.072(2)$ & $163(2)$ & - & - \\
\hline $\mathrm{O}(4)-\mathrm{H}--\mathrm{Cl}(5)$ & $3.122(2)$ & 161(2) & - & - \\
\hline $\mathrm{O}(5)-\mathrm{H}---\mathrm{Cl}(2)$ & $3.201(3)$ & $139(4)$ & - & - \\
\hline $\mathrm{O}(5)-\mathrm{H}---\mathrm{Cl}(5)$ & $3.118(3)$ & $168(4)$ & - & - \\
\hline $\mathrm{N}(1)-\mathrm{H}---\mathrm{Cl}(6)$ & $3.161(2)$ & $175.2(18)$ & - & - \\
\hline $\mathrm{C}(12)-\mathrm{H}$--- $\mathrm{C}_{\mathrm{g} 1}$ & - & - & $3.422(4)$ & 13.05 \\
\hline
\end{tabular}

Table S3. Selected noncovalent interactions - such as hydrogen bonding, halogen $-\pi, \mathrm{C}-\mathrm{H}-\pi$, and $\pi-\pi$ stacking interactions - observed in $\left[\mathrm{Th}\left(\mathrm{H}_{2} \mathrm{O}\right)_{4} \mathrm{Cl}_{4}\right] \cdot 2(\mathrm{PhenH} \cdot \mathrm{Cl}) \cdot 2 \mathrm{H}_{2} \mathrm{O}(3) \cdot \mathrm{Cg}_{\mathrm{g} 1}=$ centroid formed from ring containing atoms $\mathrm{N}_{1}, \mathrm{C}_{1}, \mathrm{C}_{2}, \mathrm{C}_{3}, \mathrm{C}_{4}, \mathrm{C}_{5} ; \mathrm{C}_{22}=$ centroid formed from ring containing atoms $\mathrm{N}_{2}, \mathrm{C}_{9}, \mathrm{C}_{8}, \mathrm{C}_{10}, \mathrm{C}_{11}, \mathrm{C}_{12} ; \mathrm{C}_{93}=$ centroid formed from ring containing atoms $\mathrm{C}_{4}$, $\mathrm{C}_{5}, \mathrm{C}_{9}, \mathrm{C}_{8}, \mathrm{C}_{7}, \mathrm{C}_{6}$.

\begin{tabular}{|c|c|c|c|c|}
\hline Interaction & $\begin{array}{c}\text { Distance }(\AA), \\
\text { D-H---A }\end{array}$ & $\begin{array}{l}\text { Angle }\left(^{\circ}\right), \\
\angle \mathrm{D}-\mathrm{H}---\mathrm{A}\end{array}$ & $\begin{array}{c}\text { Distance }(\AA), \\
C_{g}---C_{g}\end{array}$ & $\beta\left({ }^{\circ}\right)$ \\
\hline $\mathrm{O}(1)-\mathrm{H}---\mathrm{Cl}(3)$ & $3.095(4)$ & $169(4)$ & - & - \\
\hline $\mathrm{O}(1)-\mathrm{H}$-- $\mathrm{O}(3)$ & $2.781(6)$ & $173(7)$ & - & - \\
\hline $\mathrm{O}(2)-\mathrm{H}$--- $\mathrm{Cl}(1)$ & $3.083(5)$ & $156(4)$ & - & - \\
\hline $\begin{array}{l}\text { intra } \\
\mathrm{O}(2)-\mathrm{H}---\mathrm{Cl}(2)\end{array}$ & $3.003(5)$ & $100(4)$ & - & - \\
\hline $\mathrm{O}(2)-\mathrm{H}$--- $\mathrm{Cl}(3)$ & $3.033(5)$ & $128(4)$ & - & - \\
\hline $\mathrm{O}(3)-\mathrm{H}---\mathrm{Cl}(3)$ & $3.146(4)$ & $170(6)$ & - & - \\
\hline $\mathrm{O}(3)-\mathrm{H}---\mathrm{Cl}(3)$ & $3.178(5)$ & $173(5)$ & - & - \\
\hline $\mathrm{N}(2)-\mathrm{H}$--- $\mathrm{O}(3)$ & $2.801(6)$ & $151(6)$ & - & - \\
\hline $\begin{array}{c}\text { intra } \\
\mathrm{N}(2)-\mathrm{H}---\mathrm{N}(1)\end{array}$ & $2.748(9)$ & $108(4)$ & - & - \\
\hline $\mathrm{C}_{\mathrm{g} 1}---\mathrm{Cg}_{\mathrm{g} 2}$ & - & - & $3.653(4)$ & 22.8 \\
\hline $\mathrm{C}_{\mathrm{g} 1---} \mathrm{C}_{\mathrm{g} 2}$ & - & - & $3.691(4)$ & 24.1 \\
\hline $\mathrm{C}_{\mathrm{g} 2}---\mathrm{C}_{\mathrm{g} 3}$ & - & - & $3.492(4)$ & 15.2 \\
\hline $\mathrm{C}_{\mathrm{g} 2}---\mathrm{C}_{\mathrm{g} 3}$ & - & - & $3.508(4)$ & 16.4 \\
\hline $\mathrm{C}_{\mathrm{g} 3}---\mathrm{C}_{\mathrm{g} 3}$ & - & - & $3.745(4)$ & 25.7 \\
\hline
\end{tabular}


Table S4. Selected noncovalent interactions - such as hydrogen bonding, halogen $-\pi, \mathrm{C}-\mathrm{H}-\pi$, and $\pi-\pi$ stacking interactions - observed in $\left[\mathrm{Th}_{(}\left(\mathrm{H}_{2} \mathrm{O}\right)_{4} \mathrm{Cl}_{4}\right] \cdot 2\left(\mathrm{TerpyH}_{2} \cdot 2 \mathrm{Cl}\right) \cdot 3 \mathrm{H}_{2} \mathrm{O}(\mathbf{4}) \cdot \mathrm{C}_{\mathrm{g} 1}=$ centroid formed from ring containing atoms $\mathrm{N}_{1}, \mathrm{C}_{1}, \mathrm{C}_{2}, \mathrm{C}_{3}, \mathrm{C}_{4}, \mathrm{C}_{5} ; \mathrm{C}_{\mathrm{g} 2}=$ centroid formed from ring containing atoms $\mathrm{N}_{2}, \mathrm{C}_{6}, \mathrm{C}_{7}, \mathrm{C}_{8}, \mathrm{C}_{9}, \mathrm{C}_{10} ; \mathrm{C}_{94}=$ centroid formed from ring containing atoms $\mathrm{N}_{4}$, $\mathrm{C}_{16}, \mathrm{C}_{17}, \mathrm{C}_{18}, \mathrm{C}_{19}, \mathrm{C}_{20} ; \mathrm{C}_{\mathrm{g} 5}=$ centroid formed from ring containing atoms $\mathrm{N}_{5}, \mathrm{C}_{21}, \mathrm{C}_{22}, \mathrm{C}_{23}, \mathrm{C}_{24}$, $\mathrm{C}_{25}$.

\begin{tabular}{|c|c|c|c|c|}
\hline Interaction & $\begin{array}{c}\text { Distance }(\AA), \\
\text { D-H---A }\end{array}$ & $\begin{array}{l}\text { Angle }\left(^{\circ}\right), \\
\angle \mathrm{D}-\mathrm{H}---\mathrm{A}\end{array}$ & $\begin{array}{c}\text { Distance }(\AA), \\
C_{\mathrm{g}---} C_{g}\end{array}$ & $\beta\left(^{\circ}\right)$ \\
\hline $\mathrm{O}(1)-\mathrm{H}---\mathrm{Cl}(5)$ & $3.100(4)$ & $170(4)$ & - & - \\
\hline $\mathrm{O}(1)-\mathrm{H}---\mathrm{Cl}(6)$ & $3.141(4)$ & $165(4)$ & - & - \\
\hline $\begin{array}{l}\text { intra } \\
\mathrm{O}(2)-\mathrm{H} \text {--- } \mathrm{Cl}(1)\end{array}$ & $2.939(4)$ & $107(3)$ & - & - \\
\hline $\mathrm{O}(2)-\mathrm{H}---\mathrm{Cl}(3)$ & $3.405(3)$ & $154(4)$ & - & - \\
\hline $\mathrm{O}(2)-\mathrm{H}$--- $\mathrm{O}(5)$ & $2.639(5)$ & $170(6)$ & - & - \\
\hline $\mathrm{O}(3)-\mathrm{H}---\mathrm{Cl}(6)$ & $3.055(4)$ & $173(4)$ & - & - \\
\hline $\mathrm{O}(3)-\mathrm{H}---\mathrm{Cl}(5)$ & $3.066(4)$ & $159(5)$ & - & - \\
\hline $\begin{array}{c}\text { intra } \\
\mathrm{O}(4)-\mathrm{H} \text {--- } \mathrm{Cl}(4)\end{array}$ & $3.045(4)$ & $110(3)$ & - & - \\
\hline $\mathrm{O}(4)-\mathrm{H}--\mathrm{O}(6)$ & $2.676(5)$ & $156(5)$ & - & - \\
\hline $\mathrm{O}(4)-\mathrm{H}$--- O(7) & $2.669(7)$ & $175(6)$ & - & - \\
\hline $\mathrm{O}(5)-\mathrm{H}---\mathrm{Cl}(5)$ & $3.245(7)$ & $156(5)$ & - & - \\
\hline $\mathrm{O}(5)-\mathrm{H}---\mathrm{Cl}(6)$ & $3.176(4)$ & $169(6)$ & - & - \\
\hline $\mathrm{O}(6)-\mathrm{H}---\mathrm{Cl}(7)$ & $3.097(4)$ & $170(4)$ & - & - \\
\hline $\mathrm{O}(6)-\mathrm{H}---\mathrm{Cl}(8)$ & $3.160(4)$ & $155(5)$ & - & - \\
\hline $\mathrm{N}(1)-\mathrm{H}--\mathrm{Cl}(8)$ & $3.026(5)$ & $155(5)$ & - & - \\
\hline $\begin{array}{c}\text { intra } \\
\mathrm{N}(1)-\mathrm{H}---\mathrm{N}(2)\end{array}$ & $2.678(7)$ & $102(4)$ & - & - \\
\hline $\mathrm{N}(3)-\mathrm{H}---\mathrm{Cl}(8)$ & $3.083(6)$ & $165(6)$ & - & - \\
\hline $\mathrm{N}(4)-\mathrm{H}---\mathrm{Cl}(7)$ & $3.101(6)$ & $160(5)$ & - & - \\
\hline $\begin{array}{c}\text { intra } \\
\mathrm{N}(4)-\mathrm{H}---\mathrm{N}(5)\end{array}$ & $2.662(7)$ & $100(4)$ & - & - \\
\hline $\mathrm{N}(6)-\mathrm{H}---\mathrm{Cl}(7)$ & $3.059(5)$ & $163(5)$ & - & - \\
\hline $\mathrm{Cg}_{\mathrm{g} 1---} \mathrm{C}_{\mathrm{g} 5}$ & - & - & $3.884(3)$ & 31.5 \\
\hline $\mathrm{C}_{\mathrm{g} 2---} \mathrm{C}_{\mathrm{g} 4}$ & - & - & $3.826(3)$ & 30.3 \\
\hline
\end{tabular}


Table S5. Selected noncovalent interactions - such as hydrogen bonding, halogen $-\pi, \mathrm{C}-\mathrm{H}-\pi$, and $\pi-\pi$ stacking interactions - observed in $\left[\mathrm{Th}\left(\mathrm{H}_{2} \mathrm{O}\right)_{4} \mathrm{Cl}_{4}\right] \cdot 2(4-\mathrm{ClPyH} \cdot \mathrm{Cl})(\mathbf{5}) \cdot \mathrm{C}_{\mathrm{g} 1}=$ centroid formed from ring containing atoms $\mathrm{N}_{1}, \mathrm{C}_{1}, \mathrm{C}_{2}, \mathrm{C}_{3}, \mathrm{C}_{2}, \mathrm{C}_{1} ; \mathrm{C}_{\mathrm{g} 2}=$ centroid formed from ring containing atoms $\mathrm{N}_{2}, \mathrm{C}_{4}, \mathrm{C}_{5}, \mathrm{C}_{6}, \mathrm{C}_{7}, \mathrm{C}_{8} ; \mathrm{C}_{\mathrm{g} 3}=$ centroid formed from ring containing atoms $\mathrm{N}_{2 \mathrm{~B}}, \mathrm{C}_{4 \mathrm{~B}}, \mathrm{C}_{5 \mathrm{~B}}, \mathrm{C}_{6 \mathrm{~B}}$, $\mathrm{C}_{7 \mathrm{~B}}, \mathrm{C}_{8 \mathrm{~B}}$.

\begin{tabular}{ccccc}
\hline Interaction & $\begin{array}{c}\text { Distance }(\AA), \\
\mathrm{D}-\mathrm{H}---\mathrm{A}\end{array}$ & $\begin{array}{c}\text { Angle }\left({ }^{\circ}\right), \\
\angle \mathrm{D}-\mathrm{H}---\mathrm{A}\end{array}$ & $\begin{array}{c}\text { Distance }(\AA), \\
\mathrm{C}_{\mathrm{g}---\mathrm{C}_{\mathrm{g}}}\end{array}$ & $\begin{array}{c}\beta \text { or } \gamma \\
\left({ }^{\circ}\right)\end{array}$ \\
\hline $\mathrm{O}(1)-\mathrm{H}--\mathrm{Cl}(3)$ & $3.066(5)$ & $173(2)$ & - & - \\
$\mathrm{N}(1)-\mathrm{H}--\mathrm{Cl}(2)$ & $3.421(2)$ & $129(2)$ & - & - \\
$\mathrm{N}(1)-\mathrm{H}---\mathrm{O}(1)$ & $2.965(4)$ & $143(5)$ & - & - \\
$\mathrm{O}(2)-\mathrm{H}--\mathrm{Cl}(1)$ & $3.129(2)$ & $154(2)$ & - & - \\
$\mathrm{O}(3)-\mathrm{H}--\mathrm{Cl}(3)$ & $3.038(4)$ & $174(3)$ & - & - \\
$\mathrm{O}(3)-\mathrm{H}--\mathrm{Cl}(3)$ & $3.184(6)$ & $165(2)$ & - & - \\
$\mathrm{Cg} 2--\mathrm{Cl}(4)$ & - & - & $3.615(5)$ & 8.19 \\
$\mathrm{Cg}_{\mathrm{g} 3}--\mathrm{Cl}(4)$ & - & - & $3.417(7)$ & 27.88 \\
$\mathrm{Cg}_{\mathrm{g} 1}-\mathrm{Cl}(5)$ & - & - & $3.586(5)$ & 8.67 \\
$\mathrm{Cg}_{\mathrm{g} 1--\mathrm{C} 2}$ & - & - & $3.787(4)$ & 11.3 \\
$\mathrm{Cg}_{\mathrm{g} 1--} \mathrm{Cg}_{\mathrm{g} 3}$ & - & - & $3.548(6)$ & 12.2 \\
$\mathrm{Cg}_{\mathrm{g} 1--} \mathrm{Cg}_{\mathrm{g} 3}$ & - & & $3.589(6)$ & 18.4
\end{tabular}




\section{COMPUTATIONAL DETAILS.}

Table S6. Reaction energies $\Delta \mathrm{G}_{a q}$ in $\mathrm{kJ} / \mathrm{mol}$ at the B3LYP/DZVP2 and B3LYP/aD DFT levels and CCSD $(\mathrm{T}) / \mathrm{aD}(\mathrm{aD}=$ aug-ccpVDZ(H,O)/aug-cc-pV(D+d)Z(Cl)/cc-pVDZ-PP(Th)) and MP2/aD correlated MO levels. Opt at B3LYP/DZVP2 in gas or in solution.

\begin{tabular}{|c|c|c|c|c|c|c|c|c|}
\hline Optimization level & gas & gas & $\mathrm{aq}$ & $\mathrm{aq}$ & gas & gas & $\mathrm{aq}$ & $\mathrm{aq}$ \\
\hline method & B3LYP & B3LYP & B3LYP & B3LYP & $\operatorname{CCSD}(\mathrm{T})$ & MP2 & $\operatorname{CCSD}(\mathrm{T})$ & MP2 \\
\hline Basis set & DZVP2 & $\mathrm{aD}$ & DZVP2 & $\mathrm{aD}$ & $\mathrm{aD}$ & $\mathrm{aD}$ & $\mathrm{aD}$ & $\mathrm{aD}$ \\
\hline \multicolumn{9}{|l|}{ Reaction } \\
\hline $\mathrm{Th}\left(\mathrm{H}_{2} \mathrm{O}\right)_{9}{ }^{4+}+\mathrm{Cl}^{-} \rightarrow \mathrm{Th}\left(\mathrm{H}_{2} \mathrm{O}\right)_{8} \mathrm{Cl}^{3+}+\mathrm{H}_{2} \mathrm{O}$ & -44.4 & -36.8 & -15.1 & -4.2 & -41.8 & -40.2 & -13.0 & -9.2 \\
\hline $\begin{array}{l}\mathrm{Th}\left(\mathrm{H}_{2} \mathrm{O}\right)_{8} \mathrm{Cl}^{3+}+\mathrm{Cl}^{-} \rightarrow \mathrm{Th}\left(\mathrm{H}_{2} \mathrm{O}\right)_{7} \mathrm{Cl}_{2}{ }^{2+}(\mathbf{g})+ \\
\mathrm{H}_{2} \mathrm{O}\end{array}$ & -52.3 & -43.5 & -47.3 & -37.7 & -51.9 & -53.1 & -44.8 & -46.0 \\
\hline $\begin{array}{l}\mathrm{Th}\left(\mathrm{H}_{2} \mathrm{O}\right)_{7} \mathrm{Cl}_{2}{ }^{2+}(\mathbf{g})+\mathrm{Cl}^{-} \rightarrow \mathrm{Th}\left(\mathrm{H}_{2} \mathrm{O}\right)_{6} \mathrm{Cl}_{3}{ }^{1+}(\mathbf{f})+ \\
\mathrm{H}_{2} \mathrm{O}\end{array}$ & -26.4 & -14.2 & -23.0 & -10.0 & -22.6 & -23.4 & -14.2 & -14.6 \\
\hline $\begin{array}{l}\mathrm{Th}\left(\mathrm{H}_{2} \mathrm{O}\right)_{6} \mathrm{Cl}_{3}{ }^{1+}(\mathbf{f})+\mathrm{Cl}^{-} \rightarrow \mathrm{Th}\left(\mathrm{H}_{2} \mathrm{O}\right)_{5} \mathrm{Cl}_{4}(\mathbf{e})+ \\
\mathrm{H}_{2} \mathrm{O}\end{array}$ & -14.6 & -5.9 & -10.0 & 0.8 & -11.3 & -13.8 & -5.4 & -7.5 \\
\hline $\begin{array}{l}\mathrm{Th}\left(\mathrm{H}_{2} \mathrm{O}\right)_{5} \mathrm{Cl}_{4}(\mathbf{e})+\mathrm{Cl}^{-} \rightarrow \mathrm{Th}\left(\mathrm{H}_{2} \mathrm{O}\right)_{3} \mathrm{Cl}_{5}{ }^{1-}+2 \\
\mathrm{H}_{2} \mathrm{O}\end{array}$ & -26.4 & -21.3 & -36.0 & -29.7 & -2.1 & -3.8 & -8.8 & -11.7 \\
\hline $\begin{array}{l}\mathrm{Th}\left(\mathrm{H}_{2} \mathrm{O}\right)_{3} \mathrm{Cl}_{5}{ }^{1-}+\mathrm{Cl}^{-} \rightarrow \mathrm{Th}\left(\mathrm{H}_{2} \mathrm{O}\right)_{2} \mathrm{Cl}_{6}{ }^{2-}(\mathbf{a})+ \\
\mathrm{H}_{2} \mathrm{O}\end{array}$ & & & 13.4 & 24.7 & & & 22.6 & 19.2 \\
\hline $\mathrm{Th}\left(\mathrm{H}_{2} \mathrm{O}\right)_{5} \mathrm{Cl}_{4}(\mathbf{e}) \rightarrow \mathrm{Th}\left(\mathrm{H}_{2} \mathrm{O}\right)_{4} \mathrm{Cl}_{4}(\mathbf{b})+\mathrm{H}_{2} \mathrm{O}$ & -34.7 & -37.2 & -23.4 & -27.2 & -14.6 & -13.8 & -2.1 & -1.7 \\
\hline $\mathrm{Th}\left(\mathrm{H}_{2} \mathrm{O}\right)_{5} \mathrm{Cl}_{4}(\mathbf{e}) \rightarrow \mathrm{Th}\left(\mathrm{H}_{2} \mathrm{O}\right)_{4} \mathrm{Cl}_{4}(\mathbf{c})+\mathrm{H}_{2} \mathrm{O}$ & -32.2 & -34.7 & -25.5 & -29.7 & -12.1 & -11.3 & -4.6 & -4.6 \\
\hline $\mathrm{Th}\left(\mathrm{H}_{2} \mathrm{O}\right)_{5} \mathrm{Cl}_{4}(\mathbf{e}) \rightarrow \mathrm{Th}\left(\mathrm{H}_{2} \mathrm{O}\right)_{4} \mathrm{Cl}_{4}(\mathbf{d})+\mathrm{H}_{2} \mathrm{O}$ & -20.1 & -23.0 & -22.6 & -27.2 & 4.2 & 5.0 & 1.3 & 1.7 \\
\hline $\mathrm{Th}\left(\mathrm{H}_{2} \mathrm{O}\right)_{4} \mathrm{Cl}_{4}(\mathbf{b})+\mathrm{Cl}^{-} \rightarrow \mathrm{Th}\left(\mathrm{H}_{2} \mathrm{O}\right)_{3} \mathrm{Cl}_{5}{ }^{1-}+\mathrm{H}_{2} \mathrm{O}$ & 8.4 & 15.9 & -12.6 & -2.5 & 12.6 & 10.0 & -6.7 & -9.6 \\
\hline $\mathrm{Th}\left(\mathrm{H}_{2} \mathrm{O}\right)_{4} \mathrm{Cl}_{4}(\mathbf{c})+\mathrm{Cl}^{-} \rightarrow \mathrm{Th}\left(\mathrm{H}_{2} \mathrm{O}\right)_{3} \mathrm{Cl}_{5}{ }^{1-}+\mathrm{H}_{2} \mathrm{O}$ & 5.9 & 13.4 & -10.5 & 0.0 & 10.0 & 7.5 & -4.6 & -7.1 \\
\hline $\mathrm{Th}\left(\mathrm{H}_{2} \mathrm{O}\right)_{4} \mathrm{Cl}_{4}(\mathbf{d})+\mathrm{Cl}^{-} \rightarrow \mathrm{Th}\left(\mathrm{H}_{2} \mathrm{O}\right)_{3} \mathrm{Cl}_{5}{ }^{1-}+\mathrm{H}_{2} \mathrm{O}$ & -6.3 & 1.7 & -13.4 & -2.5 & -6.7 & -8.8 & -10.0 & -13.4 \\
\hline
\end{tabular}


Table S7. Reaction energies gas phase in kJ/mol at the B3LYP/DZVP2/cc-pVDZ-PP DFT levels and CCSD(T)/aD and MP2/aD correlated MO levels.

\begin{tabular}{|c|c|c|c|c|c|c|c|}
\hline \multirow[t]{2}{*}{ Reaction } & \multicolumn{2}{|c|}{ B3LYP/DZVP2 } & \multicolumn{2}{|c|}{$\mathrm{CCSD}(\mathrm{T}) / \mathrm{aD}$} & \multicolumn{2}{|c|}{$\mathrm{MP} 2 / \mathrm{aD}$} & \multirow{2}{*}{$\Delta$ Gsolv* } \\
\hline & $\Delta \mathrm{H}$ & $\Delta \mathrm{G}$ & $\Delta \mathrm{H}$ & $\Delta \mathrm{G}$ & $\Delta \mathrm{H}$ & $\Delta \mathrm{G}$ & \\
\hline $\mathrm{Th}\left(\mathrm{H}_{2} \mathrm{O}\right){ }_{9}^{4+}+\mathrm{Cl}^{-} \rightarrow \mathrm{Th}\left(\mathrm{H}_{2} \mathrm{O}\right)_{8} \mathrm{Cl}^{3+}+\mathrm{H}_{2} \mathrm{O}$ & -1490.3 & -1513.4 & -1479.5 & -1502.5 & -1477.8 & -1500.8 & 1442.6 \\
\hline $\mathrm{Th}\left(\mathrm{H}_{2} \mathrm{O}\right)_{8} \mathrm{Cl}^{3+}+\mathrm{Cl}^{-} \rightarrow \mathrm{Th}\left(\mathrm{H}_{2} \mathrm{O}\right)_{7} \mathrm{Cl}_{2}^{2+}(\mathrm{g})+\mathrm{H}_{2} \mathrm{O}$ & -1175.3 & -1182.4 & -1165.2 & -1172.4 & -1166.1 & -1173.2 & 1102.1 \\
\hline $\mathrm{Th}\left(\mathrm{H}_{2} \mathrm{O}\right)_{7} \mathrm{Cl}_{2}{ }^{2+}(\mathbf{g})+\mathrm{Cl}^{-} \rightarrow \mathrm{Th}\left(\mathrm{H}_{2} \mathrm{O}\right)_{6} \mathrm{Cl}_{3}{ }^{1+}(\mathbf{f})+\mathrm{H}_{2} \mathrm{O}$ & -809.2 & -816.3 & -792.0 & -799.6 & -792.9 & -800.4 & 759.0 \\
\hline $\mathrm{Th}\left(\mathrm{H}_{2} \mathrm{O}\right)_{6} \mathrm{Cl}_{3}{ }^{1+}(\mathbf{f})+\mathrm{Cl}^{-} \rightarrow \mathrm{Th}\left(\mathrm{H}_{2} \mathrm{O}\right)_{5} \mathrm{Cl}_{4}(\mathbf{e})+\mathrm{H}_{2} \mathrm{O}$ & -492.5 & -500.8 & -476.1 & -484.5 & -478.2 & -486.6 & 454.8 \\
\hline $\mathrm{Th}\left(\mathrm{H}_{2} \mathrm{O}\right)_{5} \mathrm{Cl}_{4}(\mathbf{e}) \rightarrow \mathrm{Th}\left(\mathrm{H}_{2} \mathrm{O}\right)_{4} \mathrm{Cl}_{4}(\mathbf{b})+\mathrm{H}_{2} \mathrm{O}$ & 18.4 & -30.1 & 33.5 & -15.1 & 34.3 & -14.2 & -17.6 \\
\hline $\mathrm{Th}\left(\mathrm{H}_{2} \mathrm{O}\right)_{5} \mathrm{Cl}_{4}(\mathbf{e}) \rightarrow \mathrm{Th}\left(\mathrm{H}_{2} \mathrm{O}\right)_{4} \mathrm{Cl}_{4}(\mathbf{c})+\mathrm{H}_{2} \mathrm{O}$ & 18.8 & -28.0 & 33.5 & -13.0 & 34.3 & -12.1 & -17.2 \\
\hline $\mathrm{Th}\left(\mathrm{H}_{2} \mathrm{O}\right)_{5} \mathrm{Cl}_{4}(\mathbf{e}) \rightarrow \mathrm{Th}\left(\mathrm{H}_{2} \mathrm{O}\right)_{4} \mathrm{Cl}_{4}(\mathbf{d})+\mathrm{H}_{2} \mathrm{O}$ & 43.1 & -2.9 & 63.6 & 17.2 & 64.0 & 18.0 & -31.0 \\
\hline $\mathrm{Th}\left(\mathrm{H}_{2} \mathrm{O}\right)_{4} \mathrm{Cl}_{4}(\mathbf{b})+\mathrm{Cl}^{-} \rightarrow \mathrm{Th}\left(\mathrm{H}_{2} \mathrm{O}\right)_{3} \mathrm{Cl}_{5}{ }^{1-}+\mathrm{H}_{2} \mathrm{O}$ & -172.4 & -174.1 & -154.0 & -155.6 & -156.5 & -158.2 & 150.2 \\
\hline $\mathrm{Th}\left(\mathrm{H}_{2} \mathrm{O}\right)_{4} \mathrm{Cl}_{4}(\mathbf{c})+\mathrm{Cl}^{-} \rightarrow \mathrm{Th}\left(\mathrm{H}_{2} \mathrm{O}\right)_{3} \mathrm{Cl}_{5}{ }^{1-}+\mathrm{H}_{2} \mathrm{O}$ & -172.4 & -176.1 & -154.0 & -157.7 & -156.5 & -160.2 & 149.8 \\
\hline $\mathrm{Th}\left(\mathrm{H}_{2} \mathrm{O}\right)_{4} \mathrm{Cl}_{4}(\mathbf{d})+\mathrm{Cl}^{-} \rightarrow \mathrm{Th}\left(\mathrm{H}_{2} \mathrm{O}\right)_{3} \mathrm{Cl}_{5}{ }^{1-}+\mathrm{H}_{2} \mathrm{O}$ & -197.1 & -201.3 & -183.7 & -188.3 & -186.2 & -190.4 & 163.6 \\
\hline
\end{tabular}

$* \Delta \mathrm{G}_{\text {solv }}$ at B3LYP/DZVP2

Table S8. Frequencies $\left(\mathrm{cm}^{-1}\right)$, IR Intensities (km/mole) and Raman Activities $\left(\AA^{4} / \mathrm{amu}\right)$ at B3LYP/DZVP2/cc-pVDZ-PP level.

\begin{tabular}{|c|c|c|c|c|c|c|c|c|c|c|c|c|c|c|}
\hline \multicolumn{3}{|c|}{$\mathrm{Th}\left(\mathrm{H}_{2} \mathrm{O}\right)_{9}{ }^{4+}$} & \multicolumn{3}{|c|}{$\mathrm{Th}\left(\mathrm{H}_{2} \mathrm{O}\right)_{8} \mathrm{Cl}^{3+}$} & \multicolumn{3}{|c|}{$\mathrm{Th}\left(\mathrm{H}_{2} \mathrm{O}\right)_{7} \mathrm{Cl}_{2}{ }^{2+}(\mathbf{g})$} & \multicolumn{3}{|c|}{$\mathrm{Th}\left(\mathrm{H}_{2} \mathrm{O}\right)_{6} \mathrm{Cl}_{3}{ }^{1+}(\mathbf{f})$} & \multicolumn{3}{|c|}{$\mathrm{Th}\left(\mathrm{H}_{2} \mathrm{O}\right)_{5} \mathrm{Cl}_{4}(\mathbf{e})$} \\
\hline Freq & IR & Raman & Freq & Int & Raman & Freq & Int & Raman & Freq & Int & Raman & Freq & Int & Raman \\
\hline 48.0 & 0.1 & 0.0 & 16.8 & 3.7 & 0.0 & 22.1 & 8.2 & 0.1 & 37.2 & 2.0 & 0.2 & 34.4 & 4.6 & 0.0 \\
\hline 48.0 & 0.1 & 0.0 & 62.2 & 0.2 & 0.0 & 64.5 & 2.3 & 0.2 & 62.5 & 2.6 & 0.3 & 69.4 & 3.3 & 0.2 \\
\hline 102.8 & 2.4 & 0.0 & 73.8 & 1.5 & 0.1 & 68.1 & 0.2 & 0.2 & 74.4 & 9.2 & 0.5 & 75.9 & 0.9 & 0.6 \\
\hline 102.8 & 2.4 & 0.0 & 83.5 & 0.4 & 0.2 & 93.3 & 5.8 & 0.1 & 75.3 & 8.5 & 0.4 & 80.3 & 9.8 & 0.0 \\
\hline 111.0 & 0.0 & 0.0 & 87.3 & 1.6 & 0.1 & 97.9 & 10.8 & 0.1 & 95.4 & 1.4 & 0.2 & 89.2 & 1.0 & 0.7 \\
\hline
\end{tabular}




\begin{tabular}{|c|c|c|c|c|c|c|c|c|c|c|c|c|c|c|}
\hline 126.2 & 2.5 & 0.0 & 102.9 & 5.0 & 0.2 & 107.8 & 0.2 & 0.2 & 101.4 & 0.2 & 0.1 & 93.2 & 5.1 & 0.4 \\
\hline 126.7 & 2.1 & 0.0 & 110.3 & 2.8 & 0.6 & 111.9 & 1.3 & 0.4 & 108.0 & 3.8 & 0.3 & 98.4 & 2.2 & 0.2 \\
\hline 126.7 & 1.9 & 0.1 & 114.8 & 0.2 & 0.1 & 113.7 & 2.4 & 0.4 & 111.1 & 1.5 & 0.3 & 103.6 & 7.8 & 0.3 \\
\hline 126.7 & 0.2 & 0.9 & 115.6 & 0.4 & 0.0 & 118.1 & 3.4 & 1.2 & 117.4 & 4.0 & 0.4 & 112.3 & 0.9 & 0.3 \\
\hline 154.4 & 4.9 & 0.0 & 126.2 & 3.7 & 0.3 & 127.1 & 3.8 & 0.2 & 128.4 & 26.2 & 0.3 & 117.7 & 11.9 & 0.5 \\
\hline 156.1 & 0.0 & 0.4 & 141.7 & 0.1 & 0.4 & 141.6 & 3.2 & 0.2 & 136.7 & 2.0 & 0.1 & 121.9 & 26.3 & 0.4 \\
\hline 156.1 & 0.0 & 0.4 & 147.3 & 5.3 & 0.2 & 150.8 & 0.8 & 0.4 & 149.2 & 6.8 & 0.2 & 149.9 & 8.6 & 0.3 \\
\hline 177.0 & 0.0 & 0.3 & 175.7 & 3.4 & 0.6 & 179.9 & 4.6 & 0.4 & 167.4 & 3.3 & 0.3 & 165.3 & 16.4 & 0.5 \\
\hline 237.2 & 0.0 & 0.2 & 184.8 & 3.8 & 0.4 & 191.2 & 11.4 & 0.2 & 189.9 & 13.8 & 0.2 & 202.9 & 0.8 & 0.2 \\
\hline 243.6 & 0.0 & 0.1 & 212.7 & 1.0 & 0.1 & 215.4 & 5.2 & 0.1 & 212.0 & 4.0 & 0.1 & 217.5 & 13.2 & 0.1 \\
\hline 243.6 & 0.0 & 0.1 & 215.2 & 5.1 & 0.2 & 225.4 & 0.4 & 0.3 & 230.1 & 4.8 & 0.1 & 225.7 & 15.2 & 0.3 \\
\hline 264.6 & 0.1 & 0.0 & 226.5 & 5.2 & 0.3 & 228.3 & 1.2 & 0.0 & 234.2 & 8.5 & 0.1 & 233.1 & 3.2 & 0.0 \\
\hline 282.7 & 1.7 & 0.1 & 254.9 & 4.9 & 0.2 & 269.7 & 0.5 & 0.5 & 260.4 & 21.5 & 0.4 & 264.3 & 26.9 & 0.6 \\
\hline 286.1 & 1.8 & 0.2 & 269.3 & 0.2 & 0.1 & 269.7 & 0.0 & 0.1 & 283.2 & 36.2 & 0.5 & 267.3 & 73.3 & 0.5 \\
\hline 286.1 & 1.8 & 0.2 & 278.2 & 2.1 & 0.1 & 294.5 & 0.6 & 0.1 & 286.5 & 136.3 & 0.6 & 270.9 & 40.0 & 0.6 \\
\hline 291.3 & 3.1 & 0.0 & 290.6 & 29.9 & 0.2 & 297.5 & 79.8 & 0.1 & 307.4 & 19.7 & 0.4 & 282.1 & 190.5 & 0.4 \\
\hline 299.9 & 0.0 & 0.4 & 303.0 & 2.0 & 0.3 & 327.7 & 2.7 & 11.3 & 313.5 & 32.4 & 3.5 & 302.4 & 27.1 & 11.0 \\
\hline 299.9 & 0.0 & 0.4 & 318.7 & 40.6 & 1.3 & 328.4 & 85.6 & 1.3 & 317.3 & 49.9 & 8.0 & 365.3 & 65.1 & 1.4 \\
\hline 346.4 & 0.0 & 8.5 & 343.0 & 9.6 & 1.7 & 355.3 & 79.4 & 0.4 & 326.8 & 70.5 & 1.4 & 370.4 & 113.5 & 1.5 \\
\hline 369.1 & 16.4 & 0.0 & 349.8 & 18.1 & 10.4 & 357.8 & 170.2 & 0.1 & 400.7 & 52.0 & 0.8 & 389.8 & 378.9 & 0.5 \\
\hline 395.3 & 4.4 & 1.0 & 383.6 & 41.2 & 0.6 & 397.4 & 1.9 & 1.0 & 460.5 & 158.6 & 0.6 & 505.6 & 117.9 & 0.6 \\
\hline 395.3 & 4.4 & 1.0 & 411.4 & 0.1 & 0.6 & 400.2 & 21.5 & 0.3 & 470.5 & 478.0 & 0.6 & 529.2 & 13.2 & 2.2 \\
\hline 491.1 & 0.7 & 0.0 & 424.6 & 14.9 & 0.1 & 420.4 & 526.0 & 0.6 & 481.5 & 76.8 & 0.1 & 546.1 & 404.5 & 2.8 \\
\hline
\end{tabular}




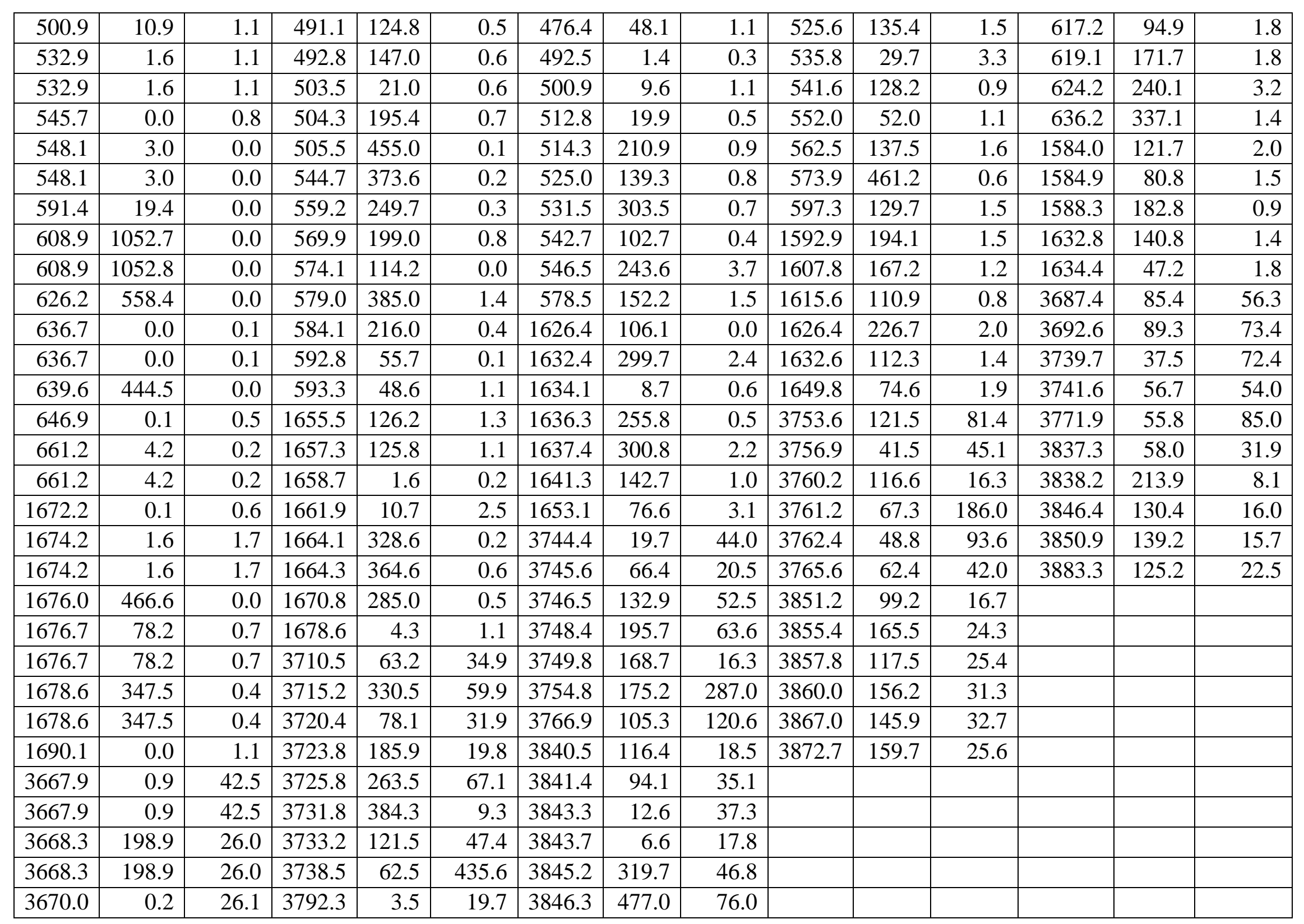




\begin{tabular}{|r|r|r|r|r|r|r|r|r|l|l|l|l|l|l|}
\hline 3671.5 & 900.9 & 0.0 & 3795.2 & 305.8 & 80.0 & 3854.3 & 190.7 & 33.3 & & & & & & \\
\hline 3675.3 & 628.4 & 14.1 & 3798.0 & 316.8 & 21.0 & & & & & & & & & \\
\hline 3675.3 & 628.4 & 14.1 & 3805.0 & 133.4 & 29.5 & & & & & & & & & \\
\hline 3691.5 & 0.0 & 635.1 & 3807.0 & 109.2 & 61.8 & & & & & & & & & \\
\hline 3730.5 & 131.0 & 0.0 & 3810.2 & 13.7 & 45.9 & & & & & & & & & \\
\hline 3731.4 & 63.2 & 40.8 & 3811.4 & 612.4 & 38.1 & & & & & & & & & \\
\hline 3731.4 & 63.2 & 40.8 & 3811.7 & 227.9 & 38.9 & & & & & & & & & \\
\hline 3732.0 & 0.0 & 60.8 & & & & & & & & & & & & \\
\hline 3732.2 & 637.1 & 10.5 & & & & & & & & & & & & \\
\hline 3732.2 & 637.1 & 10.5 & & & & & & & & & & & & \\
\hline 3737.7 & 132.2 & 48.7 & & & & & & & & & & & & \\
\hline 3737.7 & 132.2 & 48.7 & & & & & & & & & & & & \\
\hline 3738.4 & 621.0 & 0.0 & & & & & & & & & & & & \\
\hline
\end{tabular}

Table S9. Frequencies $\left(\mathrm{cm}^{-1}\right)$, IR Intensities (km/mole) and Raman Activities $\left(\AA^{4} / \mathrm{amu}\right)$ at B3LYP/DZVP2/cc-pVDZ-PP level.

\begin{tabular}{|c|c|c|c|c|c|c|c|c|c|c|c|}
\hline \multicolumn{3}{|c|}{$\mathrm{Th}\left(\mathrm{H}_{2} \mathrm{O}\right)_{4} \mathrm{Cl}_{4}(\mathbf{d})$} & \multicolumn{3}{|c|}{$\mathrm{Th}\left(\mathrm{H}_{2} \mathrm{O}\right)_{4} \mathrm{Cl}_{4}(\mathbf{c})$} & \multicolumn{3}{|c|}{$\mathrm{Th}\left(\mathrm{H}_{2} \mathrm{O}\right)_{4} \mathrm{Cl}_{4}(\mathbf{b})$} & \multicolumn{3}{|c|}{$\mathrm{Th}\left(\mathrm{H}_{2} \mathrm{O}\right)_{3} \mathrm{Cl}_{5}{ }^{1-}$} \\
\hline Freq & Int & Raman & Freq & Int & Raman & Freq & Int & Raman & Freq & Int & Raman \\
\hline 26.6 & 2.0 & 0.4 & 26.3 & 7.5 & 0.6 & 24.8 & 12.7 & 0.0 & 39.7 & 0.4 & 0.4 \\
\hline 52.2 & 2.8 & 0.1 & 26.7 & 12.1 & 0.0 & 25.3 & 7.8 & 0.7 & 48.7 & 6.5 & 0.1 \\
\hline 68.3 & 7.5 & 0.2 & 54.6 & 0.0 & 0.2 & 54.3 & 0.0 & 0.1 & 70.5 & 1.5 & 0.1 \\
\hline 84.4 & 4.3 & 0.3 & 69.7 & 0.0 & 0.9 & 70.0 & 0.0 & 1.0 & 79.4 & 0.6 & 0.4 \\
\hline 88.8 & 2.1 & 0.4 & 92.0 & 2.5 & 0.2 & 93.0 & 2.7 & 0.1 & 92.0 & 0.7 & 0.8 \\
\hline 89.4 & 0.5 & 0.3 & 100.0 & 0.0 & 0.0 & 100.2 & 0.0 & 0.0 & 102.0 & 6.7 & 0.6 \\
\hline 101.0 & 9.5 & 1.3 & 102.0 & 18.1 & 0.4 & 102.7 & 17.9 & 0.4 & 104.6 & 6.2 & 1.3 \\
\hline 105.2 & 6.5 & 1.1 & 121.0 & 7.5 & 0.1 & 120.0 & 6.1 & 0.1 & 115.0 & 0.6 & 0.5 \\
\hline 110.6 & 22.9 & 0.3 & 122.7 & 0.0 & 1.3 & 122.8 & 0.0 & 1.3 & 137.1 & 57.7 & 0.3 \\
\hline 128.0 & 6.5 & 0.4 & 134.0 & 26.0 & 1.1 & 134.4 & 27.0 & 1.1 & 143.8 & 8.6 & 0.4 \\
\hline 139.3 & 11.6 & 0.2 & 135.0 & 0.5 & 0.1 & 135.9 & 0.4 & 0.1 & 145.8 & 15.3 & 0.7 \\
\hline 148.5 & 21.6 & 0.6 & 138.8 & 76.2 & 0.2 & 138.7 & 76.4 & 0.3 & 158.7 & 12.1 & 0.2 \\
\hline
\end{tabular}




\begin{tabular}{|c|c|c|c|c|c|c|c|c|c|c|c|}
\hline 160.4 & 24.6 & 0.3 & 144.6 & 0.0 & 0.3 & 144.1 & 0.0 & 0.3 & 167.2 & 20.7 & 0.3 \\
\hline 193.0 & 10.6 & 0.2 & 190.8 & 0.2 & 1.1 & 189.3 & 0.2 & 1.0 & 190.7 & 23.2 & 0.3 \\
\hline 209.4 & 21.1 & 0.1 & 192.3 & 83.2 & 0.2 & 190.9 & 81.3 & 0.2 & 196.3 & 21.9 & 0.0 \\
\hline 215.0 & 1.6 & 0.1 & 226.2 & 21.8 & 0.0 & 226.2 & 21.1 & 0.0 & 209.8 & 14.2 & 0.5 \\
\hline 226.8 & 1.7 & 0.0 & 228.7 & 0.0 & 0.7 & 228.1 & 0.1 & 0.7 & 231.4 & 9.3 & 0.3 \\
\hline 248.1 & 22.2 & 0.6 & 234.7 & 2.4 & 0.1 & 232.8 & 2.4 & 0.1 & 244.0 & 10.2 & 0.4 \\
\hline 251.7 & 7.6 & 0.9 & 236.8 & 0.0 & 2.0 & 234.8 & 0.0 & 2.0 & 253.7 & 33.1 & 0.2 \\
\hline 268.3 & 32.3 & 1.0 & 239.3 & 0.5 & 0.0 & 239.1 & 0.3 & 0.0 & 255.5 & 75.3 & 0.2 \\
\hline 274.1 & 127.1 & 0.3 & 249.1 & 0.2 & 0.4 & 248.6 & 0.2 & 0.4 & 268.7 & 122.9 & 0.3 \\
\hline 281.7 & 81.1 & 0.3 & 268.7 & 49.0 & 0.0 & 268.6 & 50.5 & 0.0 & 270.8 & 152.4 & 0.7 \\
\hline 286.7 & 86.9 & 1.0 & 269.4 & 181.4 & 0.0 & 269.3 & 180.0 & 0.0 & 290.5 & 15.5 & 12.9 \\
\hline 307.5 & 23.7 & 12.3 & 270.4 & 121.7 & 0.0 & 270.1 & 121.8 & 0.0 & 352.2 & 115.1 & 1.1 \\
\hline 377.7 & 311.1 & 0.7 & 303.9 & 0.0 & 13.0 & 303.8 & 0.0 & 13.0 & 577.9 & 389.9 & 1.2 \\
\hline 392.1 & 125.4 & 0.4 & 452.2 & 172.8 & 0.2 & 450.5 & 173.4 & 0.2 & 598.1 & 147.1 & 3.4 \\
\hline 449.1 & 293.7 & 0.3 & 481.5 & 1399.8 & 0.0 & 479.6 & 1394.5 & 0.0 & 628.2 & 153.2 & 3.2 \\
\hline 527.2 & 142.8 & 2.1 & 494.2 & 0.7 & 0.1 & 492.7 & 0.9 & 0.1 & 647.0 & 551.2 & 1.8 \\
\hline 550.3 & 142.6 & 1.1 & 514.7 & 0.0 & 0.9 & 512.8 & 0.0 & 0.8 & 668.0 & 1.2 & 7.9 \\
\hline 559.3 & 102.7 & 2.1 & 542.6 & 0.5 & 6.5 & 541.9 & 0.5 & 6.4 & 679.4 & 113.6 & 5.8 \\
\hline 569.3 & 19.5 & 2.6 & 546.2 & 0.0 & 4.7 & 545.5 & 0.0 & 4.8 & 1596.5 & 76.1 & 1.2 \\
\hline 599.4 & 27.1 & 4.8 & 572.1 & 12.1 & 2.5 & 571.3 & 13.5 & 2.5 & 1612.7 & 98.4 & 1.7 \\
\hline 620.1 & 504.7 & 1.8 & 587.4 & 89.4 & 0.6 & 586.7 & 88.8 & 0.6 & 1627.1 & 26.5 & 2.6 \\
\hline 1585.4 & 131.3 & 3.5 & 1623.7 & 53.1 & 0.1 & 1623.6 & 53.4 & 0.2 & 3668.5 & 49.4 & 47.2 \\
\hline 1596.5 & 158.8 & 1.0 & 1627.1 & 0.0 & 7.0 & 1627.0 & 0.2 & 7.0 & 3669.2 & 63.9 & 41.8 \\
\hline 1620.8 & 122.5 & 2.2 & 1627.9 & 21.1 & 1.6 & 1627.7 & 22.3 & 1.6 & 3676.9 & 74.3 & 90.9 \\
\hline 1638.4 & 80.4 & 2.2 & 1630.1 & 327.0 & 0.3 & 1629.8 & 327.3 & 0.3 & 3759.1 & 133.1 & 10.6 \\
\hline 3692.6 & 79.6 & 53.9 & 3752.8 & 0.0 & 27.5 & 3753.3 & 2.0 & 23.0 & 3765.2 & 201.5 & 13.7 \\
\hline 3723.4 & 70.2 & 48.4 & 3752.8 & 176.4 & 1.7 & 3753.4 & 173.7 & 5.2 & 3836.9 & 100.2 & 19.4 \\
\hline 3726.9 & 44.0 & 92.8 & 3753.0 & 10.2 & 2.0 & 3753.7 & 8.6 & 6.9 & & & \\
\hline 3776.7 & 69.1 & 79.6 & 3753.8 & 0.0 & 229.9 & 3754.5 & 2.6 & 226.9 & & & \\
\hline 3831.6 & 123.6 & 21.4 & 3851.5 & 0.0 & 6.5 & 3852.2 & 0.0 & 5.4 & & & \\
\hline
\end{tabular}




\begin{tabular}{|l|r|r|r|r|r|r|r|r|r|l|l|}
\hline 3849.3 & 120.3 & 27.9 & 3851.6 & 0.1 & 2.5 & 3852.5 & 0.1 & 4.2 & & & \\
\hline 3862.1 & 150.1 & 29.1 & 3852.9 & 40.4 & 36.2 & 3853.7 & 55.7 & 35.1 & & & \\
\hline 3884.8 & 128.6 & 25.2 & 3853.6 & 457.9 & 5.3 & 3854.5 & 441.9 & 6.6 & & & \\
\hline
\end{tabular}

Table S10. Electronic energies in Hartrees at different level of theory.

\begin{tabular}{|l|c|l|l|l|l|l|}
\hline \multirow{2}{*}{ Molecule } & \multicolumn{3}{|l|}{ B3LYP/DZVP2/cc-pVDZ-PP, gas } & \multicolumn{3}{l|}{ B3LYP/DZVP2/cc-pVDZ-PP, aq } \\
\cline { 2 - 7 } & $\Delta \mathrm{H}(0 \mathrm{~K})$ & $\Delta \mathrm{H}(298 \mathrm{~K})$ & $\Delta \mathrm{G}(298 \mathrm{~K})$ & $\Delta \mathrm{H}(0 \mathrm{~K})$ & \multicolumn{1}{l|}{$\Delta(298 \mathrm{~K})$} & $\Delta \mathrm{G}(298 \mathrm{~K})$ \\
\hline $\mathrm{Th}\left(\mathrm{H}_{2} \mathrm{O}\right)_{9}{ }^{4+}$ & -1093.489194 & -1093.464509 & -1093.537756 & -1094.576892 & -1094.546067 & -1094.636599 \\
\hline $\mathrm{Th}\left(\mathrm{H}_{2} \mathrm{O}\right)_{8} \mathrm{Cl}^{3+}$ & -1477.893901 & -1477.868414 & -1477.946366 & -1478.535483 & -1478.507991 & -1478.589003 \\
\hline $\mathrm{Th}\left(\mathrm{H}_{2} \mathrm{O}\right)_{7} \mathrm{Cl}_{2}^{2+}(\mathbf{g})$ & -1862.177206 & -1862.152398 & -1862.229026 & -1862.498601 & -1862.471570 & -1862.553729 \\
\hline $\mathrm{Th}\left(\mathrm{H}_{2} \mathrm{O}\right)_{6} \mathrm{Cl}_{3}{ }^{1+}(\mathbf{f})$ & -2246.320753 & -2246.296871 & -2246.372224 & -2246.456269 & -2246.430847 & -2246.509247 \\
\hline $\mathrm{Th}\left(\mathrm{H}_{2} \mathrm{O}\right)_{5} \mathrm{Cl}_{4}(\mathbf{e})$ & -2630.343715 & -2630.320703 & -2630.395252 & -2630.408469 & -2630.384625 & -2630.459731 \\
\hline $\mathrm{Th}\left(\mathrm{H}_{2} \mathrm{O}\right)_{4} \mathrm{Cl}_{4}(\mathbf{b})$ & -2553.902974 & -2553.881857 & -2553.953497 & -2553.960136 & -2553.938674 & -2554.009869 \\
\hline $\mathrm{Th}\left(\mathrm{H}_{2} \mathrm{O}\right)_{4} \mathrm{Cl}_{4}(\mathbf{c})$ & -2553.902936 & -2553.881843 & -2553.952691 & -2553.960560 & -2553.938697 & -2554.010637 \\
\hline $\mathrm{Th}\left(\mathrm{H}_{2} \mathrm{O}\right)_{4} \mathrm{Cl}_{4}(\mathbf{d})$ & -2553.893185 & -2553.872486 & -2553.943153 & -2553.958322 & -2553.936219 & -2554.011072 \\
\hline $\mathrm{Th}\left(\mathrm{H}_{2} \mathrm{O}\right)_{3} \mathrm{Cl}_{5}{ }^{1-}$ & -2937.803218 & -2937.783816 & -2937.852109 & -2937.910810 & -2937.889996 & -2937.961409 \\
\hline $\mathrm{Th}\left(\mathrm{H}_{2} \mathrm{O}\right)_{2} \mathrm{Cl}_{6}{ }^{2-}(\mathbf{a})$ & & & & -3321.853593 & -3321.834215 & -3321.902971 \\
\hline
\end{tabular}

\begin{tabular}{|l|c|c|c|}
\hline Molecule $^{\mathrm{a}}$ & B3LYP/aD & MP2/aD & CCSD(T)/aD \\
\hline $\mathrm{Th}\left(\mathrm{H}_{2} \mathrm{O}\right)_{9}{ }^{4+}$ & -1094.667666 & -1090.713357 & -1090.846623 \\
\hline $\mathrm{Th}\left(\mathrm{H}_{2} \mathrm{O}\right)_{8} \mathrm{Cl}^{3+}$ & -1478.637342 & -1474.722756 & -1474.861735 \\
\hline $\mathrm{Th}\left(\mathrm{H}_{2} \mathrm{O}\right)_{7} \mathrm{Cl}_{2}{ }^{2+}(\mathbf{g})$ & -1862.607117 & -1858.644166 & -1858.787001 \\
\hline $\mathrm{Th}\left(\mathrm{H}_{2} \mathrm{O}\right)_{6} \mathrm{Cl}_{3}{ }^{1+}(\mathbf{f})$ & -2246.571993 & -2242.403308 & -2242.550240 \\
\hline $\mathrm{Th}\left(\mathrm{H}_{2} \mathrm{O}\right)_{5} \mathrm{Cl}_{4}(\mathbf{e})$ & -2630.532502 & -2626.050569 & -2626.200894 \\
\hline $\mathrm{Th}\left(\mathrm{H}_{2} \mathrm{O}\right)_{4} \mathrm{Cl}_{4}(\mathbf{b})$ & -2554.074161 & -2549.780657 & -2549.918054 \\
\hline $\mathrm{Th}\left(\mathrm{H}_{2} \mathrm{O}\right)_{4} \mathrm{Cl}_{4}(\mathbf{c})$ & -2554.074091 & -2549.779667 & -2549.917012 \\
\hline $\mathrm{Th}\left(\mathrm{H}_{2} \mathrm{O}\right)_{4} \mathrm{Cl}_{4}(\mathbf{d})$ & -2554.072103 & -2549.761555 & -2549.899080 \\
\hline $\mathrm{Th}\left(\mathrm{H}_{2} \mathrm{O}\right)_{3} \mathrm{Cl}_{5}{ }^{-}$ & -2938.031606 & -2933.296349 & -2933.436889 \\
\hline $\mathrm{Th}\left(\mathrm{H}_{2} \mathrm{O}\right)_{2} \mathrm{Cl}_{6}{ }^{2-}(\mathbf{a})$ & -3321.982346 & -3316.684207 & -3316.827724 \\
\hline
\end{tabular}

${ }^{a}$ Single point calculations using the B3LYP/DZVP2/cc-pVDZ-PP aq optimized geometries 


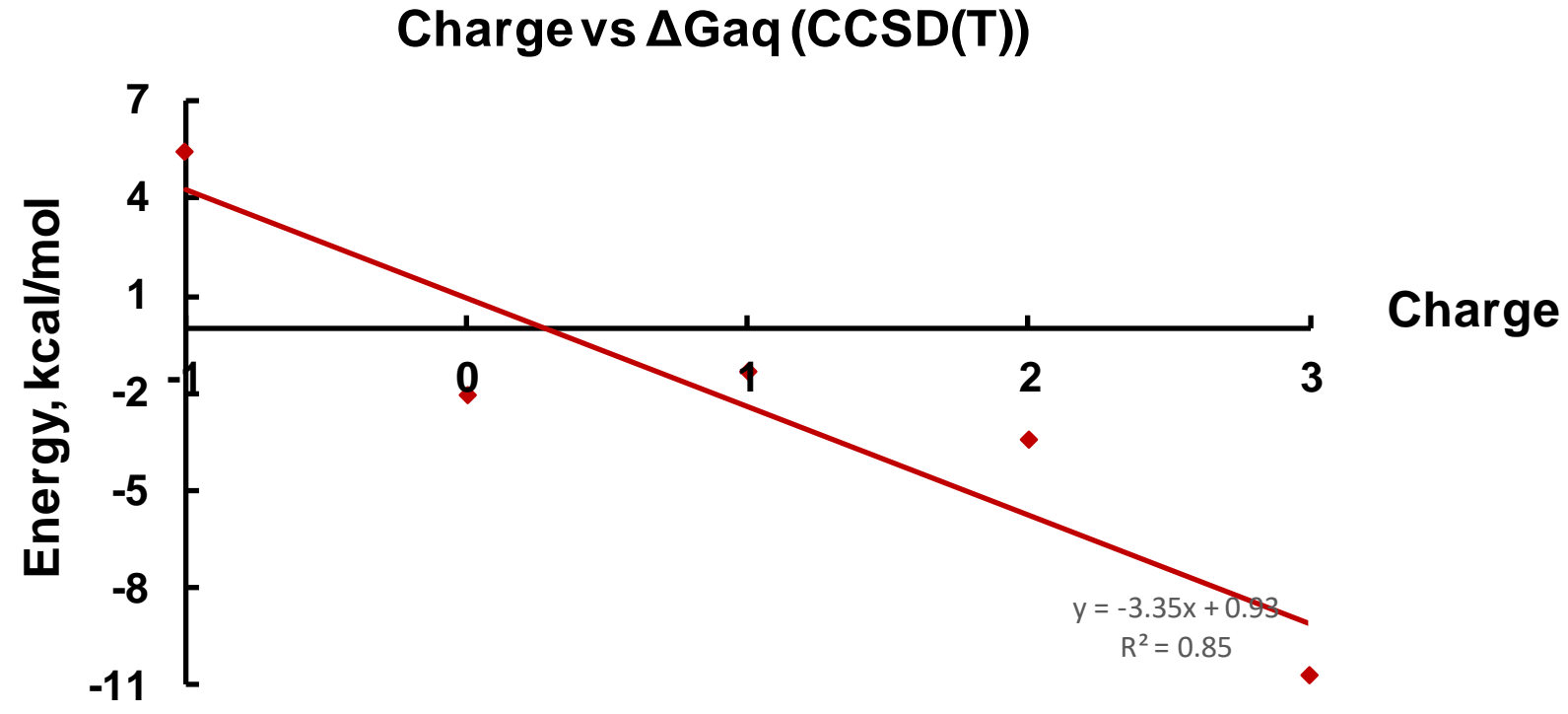

Figure S17. $\mathrm{Cl}^{-}$addition and $\mathrm{H}_{2} \mathrm{O}$ displacement energy as a function of molecular charge at the $\operatorname{CCSD}(\mathrm{T}) /$ aqueous opt level modelled for 9-coordinate $\mathrm{Th}^{\mathrm{IV}}$-aquo-chloro complexes. 
QuinoH $^{*}$<smiles>OC1NC=[NH+]c2ccccc21</smiles>

3.43
2-MePyH<smiles>Cc1cccc[nH+]1</smiles>

6.00
PhenH<smiles>c1cnc2c(c1)ccc1cccnc12</smiles>

4.84
TerpyH $\mathrm{H}_{2}$<smiles>c1ccc(-c2cccc(-c3cccc[nH+]3)n2)[nH+]c1</smiles>

$2.64,4.33$

\section{4-CIPyH 4,4'-BipyH ${ }_{2} \quad$ 3-CIPyH $\quad$ PhthalH}<smiles>Clc1cc[nH+]cc1</smiles><smiles>c1cc(-c2cc[nH+]cc2)cc[nH+]1</smiles><smiles>Clc1ccc[nH+]c1</smiles><smiles>c1ccc2c[nH+]ncc2c1</smiles>

3.83

Table S11. G3MP2 Proton Affinities $\left(\mathrm{PA}=\Delta \mathrm{H}_{298 \mathrm{~K}}\right)$ in $\mathrm{kcal} / \mathrm{mol}$ and $\mathrm{p} K$ a for Bases. ${ }^{\mathrm{a}}$

\begin{tabular}{|c|c|c|c|c|c|}
\hline Base & PA calc & PA Expt ${ }^{b}$ & $\mathrm{p} K_{\mathrm{a}}$ calc & $\begin{array}{l}\mathrm{p} K_{\text {a relative }} \\
\text { to Pyridine }\end{array}$ & $\mathrm{p} K_{\mathrm{a}} \operatorname{Expt}^{\mathrm{c}}$ \\
\hline QuinoH & $221.1 / 232.9^{\mathrm{d}}$ & & $-1.5 / 5.7^{\mathrm{d}}$ & $0.6 / 7.9^{\mathrm{d}}$ & 3.43 \\
\hline 2-МеРуH & 226.1 & 226.8 & 4.1 & 6.3 & 6.00 \\
\hline PhenH & 238.6 & & 3.3 & 5.5 & 4.84 \\
\hline 4-СlРyH & 218.8 & 219.0 & 1.3 & 3.5 & 3.83 \\
\hline 4,4'-BipyH 2 & 222.6 & & 2.1 & 4.3 & 3.17 \\
\hline 3-СlРyH & 216.7 & 215.9 & 0.2 & 2.4 & 2.81 \\
\hline PhthalH & 227.0 & & 0.8 & 3.0 & 3.47 \\
\hline TerpyH $_{2}$ (side) & 233.0 & & -0.1 & 2.1 & 2.64 \\
\hline TerpyH $_{2}$ (center) & 239.3 & & 2.9 & 5.1 & \\
\hline Pyridine & 222.1 & 222.3 & 3.0 & $5.2(\exp )$ & \\
\hline
\end{tabular}

${ }^{a}$ Removal of $\mathrm{H}^{+}$from protonated species calculated using gas phase proton affinities and COSMO solvation energies using the standard state corrected free energy for proton solvation at $298 \mathrm{~K}$ of $-264.3 \mathrm{kcal} / \mathrm{mol}^{5-6}$

${ }^{\mathrm{b}} \operatorname{Ref} 7$.

${ }^{\mathrm{c}}$ Ref 8-9.

${ }^{\mathrm{d}} \mathrm{OH}$ neutral. 
Table S12. Cartesian coordinates in $\AA$ for optimized geometries for gas phase Th complexes at the B3LYP/DZVP2(H,O,Cl)/cc-pVDZ-PP(Th) level.

$$
\begin{array}{cccc}
\mathrm{Th}\left(\mathrm{H}_{2} \mathrm{O}\right){ }_{9}{ }^{4+} & & \\
4 \mathrm{l} & & & \\
\mathrm{TH} & 0.000000 & 0.000000 & 0.000091 \\
\mathrm{O} & 0.000000 & 2.546809 & 0.000206 \\
\mathrm{H} & -0.562859 & 3.145092 & -0.534392 \\
\mathrm{O} & -2.205601 & -1.273404 & 0.000206 \\
\mathrm{H} & -2.442300 & -2.059996 & -0.534392 \\
\mathrm{O} & 2.205601 & -1.273404 & 0.000206 \\
\mathrm{H} & 3.005159 & -1.085096 & -0.534392 \\
\mathrm{H} & 2.442782 & -2.060559 & 0.533793 \\
\mathrm{H} & 0.563105 & 3.145791 & 0.533793 \\
\mathrm{H} & -3.005888 & -1.085232 & 0.533793 \\
\mathrm{O} & -0.003672 & -1.752091 & -1.833338 \\
\mathrm{H} & -0.555176 & -1.783502 & -2.643209 \\
\mathrm{O} & 1.519191 & 0.872865 & -1.833338 \\
\mathrm{H} & 1.910341 & 1.769742 & -1.898302 \\
\mathrm{O} & -1.515519 & 0.879226 & -1.833338 \\
\mathrm{H} & -1.266970 & 1.372548 & -2.643209 \\
\mathrm{H} & 0.577471 & -2.539275 & -1.898302 \\
\mathrm{H} & -2.487812 & 0.769533 & -1.898302 \\
\mathrm{H} & 1.822146 & 0.410954 & -2.643209 \\
\mathrm{O} & 0.001213 & -1.752144 & 1.833022 \\
\mathrm{H} & 0.552337 & -1.785467 & 2.643080 \\
\mathrm{O} & -1.518008 & 0.875021 & 1.833022 \\
\mathrm{H} & -1.822428 & 0.414396 & 2.643080 \\
\mathrm{O} & 1.516795 & 0.877123 & 1.833022 \\
\mathrm{H} & 1.270091 & 1.371071 & 2.643080 \\
\mathrm{H} & 2.488855 & 0.765024 & 1.897180 \\
\mathrm{H} & -1.906958 & 1.772899 & 1.897180 \\
\mathrm{H} & -0.581897 & -2.537924 & 1.897180 \\
& & & \\
\mathrm{Th}\left(\mathrm{H}_{2} \mathrm{O}\right){ }_{8} \mathrm{Cl}^{3+} & & \\
3 \mathrm{1} & & & \\
\mathrm{CL} & -2.529109 & -0.002477 & 0.000734 \\
\mathrm{H} & -1.634146 & 1.577671 & -2.171587 \\
\mathrm{H} & -0.241566 & 2.250153 & -2.370181 \\
\mathrm{H} & 0.914830 & 3.091321 & 0.394324 \\
\mathrm{H} & 2.122379 & 2.585541 & -0.445718 \\
\mathrm{H} & -0.260307 & 1.919318 & 2.687136 \\
\mathrm{H} & -1.632898 & 1.805294 & 1.961310 \\
\mathrm{H} & 0.903685 & -3.074528 & 0.531015 \\
\mathrm{H} & 2.112346 & -2.609152 & -0.330539 \\
\mathrm{H} & 2.543270 & -0.736411 & 1.973698
\end{array}
$$




$\begin{array}{cccc}\mathrm{H} & 2.544872 & 0.819480 & 1.936106 \\ \mathrm{H} & -1.633128 & -1.703566 & 2.049502 \\ \mathrm{H} & -0.257759 & -1.792349 & 2.774081 \\ \mathrm{H} & -1.632379 & -1.662319 & -2.109800 \\ \mathrm{H} & -0.240801 & -2.344533 & -2.278909 \\ \mathrm{O} & -0.698728 & 1.533794 & -1.893198 \\ \mathrm{O} & 1.326756 & 2.284787 & 0.030560 \\ \mathrm{O} & -0.678269 & 1.621253 & 1.857812 \\ \mathrm{O} & 1.317370 & -2.285829 & 0.131826 \\ \mathrm{O} & 2.078979 & 0.033325 & 1.595268 \\ \mathrm{O} & -0.678124 & -1.528600 & 1.934430 \\ \mathrm{O} & -0.696858 & -1.607310 & -1.833727 \\ \mathrm{TH} & 0.041141 & -0.000477 & -0.000994 \\ \mathrm{O} & 1.913325 & -0.043286 & -1.821601 \\ \mathrm{H} & 2.886779 & -0.041412 & -1.755605 \\ \mathrm{H} & 1.721394 & -0.064576 & -2.778836\end{array}$

$\mathrm{Th}\left(\mathrm{H}_{2} \mathrm{O}\right)_{7} \mathrm{Cl}_{2}{ }^{2+}(\mathbf{g})$

21

$\begin{array}{lrcc}\text { CL } & -2.519402 & -0.855626 & 0.015081 \\ \mathrm{CL} & 2.443135 & -0.965258 & 0.009387 \\ \mathrm{H} & -0.988005 & -2.444595 & -1.745153 \\ \mathrm{H} & 0.578986 & -2.518030 & -1.880599 \\ \mathrm{H} & 0.675462 & 0.697872 & -3.130481 \\ \mathrm{H} & 2.036705 & 0.350577 & -2.436944 \\ \mathrm{H} & -1.365245 & 2.397507 & -1.960272 \\ \mathrm{H} & -2.377660 & 1.230711 & -1.709413 \\ \mathrm{H} & 0.679085 & 0.780475 & 3.110654 \\ \mathrm{H} & 2.037760 & 0.400778 & 2.428736 \\ \mathrm{H} & 1.515740 & 2.763130 & 0.751944 \\ \mathrm{H} & 1.506985 & 2.758424 & -0.809839 \\ \mathrm{H} & -2.371525 & 1.274445 & 1.686868 \\ \mathrm{H} & -1.361612 & 2.452658 & 1.889184 \\ \mathrm{H} & -0.981481 & -2.404118 & 1.803434 \\ \mathrm{H} & 0.586262 & -2.476546 & 1.931396 \\ \mathrm{O} & -0.160594 & -1.954163 & -1.594812 \\ \mathrm{O} & 1.131752 & 0.671761 & -2.271781 \\ \mathrm{O} & -1.440449 & 1.486289 & -1.630629 \\ \mathrm{O} & 1.135727 & 0.724830 & 2.253444 \\ \mathrm{O} & 1.068545 & 2.386581 & -0.025360 \\ \mathrm{O} & -1.435239 & 1.529101 & 1.595482 \\ \mathrm{O} & -0.154349 & -1.918291 & 1.637421 \\ \mathrm{TH} & -0.000423 & 0.025365 & -0.000618\end{array}$

$\operatorname{Th}\left(\mathrm{H}_{2} \mathrm{O}\right)_{6} \mathrm{Cl}_{3}{ }^{1+}(\mathbf{f})$

11 


$\begin{array}{lccc}\text { CL } & -0.046642 & 2.597542 & -0.462442 \\ \mathrm{CL} & -2.398600 & -0.482190 & -1.069519 \\ \mathrm{CL} & 2.598973 & -0.257218 & -0.679049 \\ \mathrm{H} & -2.668852 & 1.167986 & 1.179652 \\ \mathrm{H} & -1.638734 & 1.743815 & 2.199588 \\ \mathrm{H} & 0.905228 & -2.179125 & 2.265625 \\ \mathrm{H} & 2.259536 & -1.733627 & 1.576475 \\ \mathrm{H} & -0.543065 & 0.522021 & -3.036260 \\ \mathrm{H} & 1.024033 & 0.538411 & -2.909955 \\ \mathrm{H} & -1.934155 & -1.225543 & 2.179052 \\ \mathrm{H} & 1.652504 & 0.855907 & 2.654711 \\ \mathrm{H} & -1.778272 & -2.441668 & 1.228151 \\ \mathrm{H} & 1.295751 & 2.182844 & 1.885879 \\ \mathrm{O} & -1.868957 & 0.948931 & 1.690274 \\ \mathrm{O} & -1.278170 & -1.739627 & 1.676144 \\ \mathrm{O} & 0.991064 & 1.280409 & 2.084490 \\ \mathrm{O} & 1.359763 & -1.451137 & 1.810855 \\ \mathrm{O} & 0.210904 & 0.138891 & -2.556742 \\ \mathrm{TH} & 0.022127 & 0.002374 & 0.015939 \\ \mathrm{O} & 0.133482 & -2.421993 & -0.964865 \\ \mathrm{H} & 0.985663 & -2.679834 & -1.355899 \\ \mathrm{H} & -0.549211 & -2.596954 & -1.635566\end{array}$

$\begin{array}{cccc}\mathrm{Th}\left(\mathrm{H}_{2} \mathrm{O}\right)_{5} \mathrm{Cl}_{4}(\mathbf{e}) & & \\ 01 & & & \\ \mathrm{CL} & 1.987424 & -1.673829 & -0.871403 \\ \mathrm{CL} & 1.520040 & 2.168725 & -0.687185 \\ \mathrm{CL} & -1.571961 & -2.212124 & -0.230193 \\ \mathrm{CL} & -2.113371 & 1.500399 & -0.904080 \\ \mathrm{H} & 2.636220 & 1.026362 & 1.580943 \\ \mathrm{H} & 2.933007 & -0.332554 & 0.881478 \\ \mathrm{H} & -2.800593 & 0.461910 & 1.133241 \\ \mathrm{H} & -2.395961 & -0.774472 & 1.988639 \\ \mathrm{H} & -1.060299 & -0.073856 & -2.915047 \\ \mathrm{H} & 0.149150 & -1.096593 & -2.901492 \\ \mathrm{H} & -0.894986 & 1.712933 & 2.389325 \\ \mathrm{H} & 1.164288 & -1.360398 & 2.479491 \\ \mathrm{H} & 0.082595 & 2.660632 & 1.584335 \\ \mathrm{H} & 0.122938 & -2.394218 & 1.894489 \\ \mathrm{O} & 2.357695 & 0.096632 & 1.544225 \\ \mathrm{O} & -0.031461 & 1.764907 & 1.943538 \\ \mathrm{O} & 0.257931 & -1.463372 & 2.140182 \\ \mathrm{O} & -2.149488 & 0.143363 & 1.788567 \\ \mathrm{O} & -0.181724 & -0.256709 & -2.544548 \\ \mathrm{TH} & 0.011820 & 0.017531 & -0.014583\end{array}$




$\begin{array}{lccc}\mathrm{Th}\left(\mathrm{H}_{2} \mathrm{O}\right){ }_{4} \mathrm{Cl}_{4}(\mathbf{b}) & & \\ 01 & & & \\ \mathrm{CL} & -1.430737 & 1.446558 & -1.782687 \\ \mathrm{CL} & 1.430737 & -1.446558 & -1.782687 \\ \mathrm{CL} & 2.035454 & 0.020244 & 1.782696 \\ \mathrm{CL} & -2.035454 & -0.020244 & 1.782696 \\ \mathrm{H} & 2.323485 & 1.186296 & -1.656903 \\ \mathrm{H} & 1.156552 & 2.221364 & -1.764989 \\ \mathrm{H} & -0.813890 & 2.480194 & 1.655067 \\ \mathrm{H} & 0.743427 & 2.392257 & 1.764518 \\ \mathrm{H} & 0.813890 & -2.480194 & 1.655067 \\ \mathrm{H} & -0.743427 & -2.392257 & 1.764518 \\ \mathrm{H} & -1.156552 & -2.221364 & -1.764989 \\ \mathrm{H} & -2.323485 & -1.186296 & -1.656903 \\ \mathrm{O} & 1.660551 & 1.673162 & -1.139103 \\ \mathrm{O} & 0.000000 & 2.357838 & 1.138122 \\ \mathrm{O} & 0.000000 & -2.357838 & 1.138122 \\ \mathrm{O} & -1.660551 & -1.673162 & -1.139103 \\ \mathrm{TH} & 0.000000 & 0.000000 & 0.000222\end{array}$

$\begin{array}{lrrc}\mathrm{Th}\left(\mathrm{H}_{2} \mathrm{O}\right)_{4} \mathrm{Cl}_{4}(\mathbf{c}) & & \\ 01 & & & \\ \mathrm{CL} & -1.783772 & 1.874665 & 0.789388 \\ \mathrm{CL} & -1.783772 & -1.874665 & -0.789388 \\ \mathrm{CL} & 1.783772 & -1.874665 & 0.789388 \\ \mathrm{CL} & 1.783772 & 1.874665 & -0.789388 \\ \mathrm{H} & -1.760872 & -0.216985 & 2.495968 \\ \mathrm{H} & -1.760872 & 0.216985 & -2.495968 \\ \mathrm{H} & -1.659763 & -1.690300 & 1.982471 \\ \mathrm{H} & -1.659763 & 1.690300 & -1.982471 \\ \mathrm{H} & 1.659763 & 1.690300 & 1.982471 \\ \mathrm{H} & 1.659763 & -1.690300 & -1.982471 \\ \mathrm{H} & 1.760872 & 0.216985 & 2.495968 \\ \mathrm{H} & 1.760872 & -0.216985 & -2.495968 \\ \mathrm{O} & -1.138030 & -0.894641 & 2.181054 \\ \mathrm{O} & -1.138030 & 0.894641 & -2.181054 \\ \mathrm{O} & 1.138030 & 0.894641 & 2.181054 \\ \mathrm{O} & 1.138030 & -0.894641 & -2.181054 \\ \mathrm{TH} & 0.000000 & 0.000000 & 0.000000\end{array}$

\section{$\mathrm{Th}\left(\mathrm{H}_{2} \mathrm{O}\right)_{4} \mathrm{Cl}_{4}(\mathbf{d})$}

01

$\begin{array}{llll}\text { CL } & 2.702238 & -0.027312 & 0.414103\end{array}$

$\begin{array}{llll}\text { CL } & 0.316673 & 2.606506 & -0.613612\end{array}$

$\begin{array}{llll}\text { CL } & -2.479371 & -0.006747 & -1.043226\end{array}$

$\begin{array}{llll}\text { CL } & 0.305425 & -2.584970 & -0.703382\end{array}$ 


$\begin{array}{crcc}\mathrm{H} & 1.346958 & 0.813405 & -2.683456 \\ \mathrm{H} & 1.310700 & -0.765502 & -2.700003 \\ \mathrm{H} & -2.376773 & -1.398058 & 1.482795 \\ \mathrm{H} & -1.279538 & -2.450924 & 1.155430 \\ \mathrm{H} & 0.114584 & -0.515015 & 3.118911 \\ \mathrm{H} & -2.402154 & 1.568814 & 0.930655 \\ \mathrm{H} & 1.560988 & -0.071109 & 2.636774 \\ \mathrm{H} & -1.327348 & 2.212161 & 1.858307 \\ \mathrm{O} & 0.841492 & 0.032275 & -2.406607 \\ \mathrm{O} & -1.435953 & -1.602780 & 1.615098 \\ \mathrm{O} & 0.604966 & 0.091209 & 2.541051 \\ \mathrm{O} & -1.692673 & 1.360959 & 1.566691 \\ \mathrm{TH} & 0.023839 & 0.019620 & 0.008386 \\ & & & \\ \mathrm{Th}\left(\mathrm{H}_{2} \mathrm{O}\right)_{3} \mathrm{Cl} 5^{1-} & & \\ -1 \mathrm{1} & & & \\ \mathrm{CL} & 0.129024 & 1.538543 & -2.245348 \\ \mathrm{CL} & -1.964456 & -1.388489 & -1.379631 \\ \mathrm{CL} & 2.578678 & 0.946018 & 0.430743 \\ \mathrm{CL} & -1.572319 & 2.138421 & 0.939019 \\ \mathrm{TH} & -0.018941 & -0.039481 & -0.005723 \\ \mathrm{CL} & 0.916411 & -2.367391 & 0.970247 \\ \mathrm{O} & -2.028073 & -0.806720 & 1.606284 \\ \mathrm{O} & 0.460483 & 0.563592 & 2.560298 \\ \mathrm{H} & -0.010340 & 1.415722 & 2.619322 \\ \mathrm{H} & -2.529208 & -1.330444 & 0.953154 \\ \mathrm{H} & 1.410056 & 0.775920 & 2.476916 \\ \mathrm{H} & -2.492401 & 0.047934 & 1.683974 \\ \mathrm{O} & 1.547195 & -1.084596 & -1.853668 \\ \mathrm{H} & 2.416728 & -1.165071 & -1.428804 \\ \mathrm{H} & 1.588258 & -0.309699 & -2.448326\end{array}$


Table S13. Cartesian coordinates in $\AA$ for optimized geometries for aqueous solution phase Th complexes at the B3LYP/DZVP2(H,O,Cl)/cc-pVDZ-PP(Th)/COSMO level.

$\begin{array}{cccc}\mathrm{Th}\left(\mathrm{H}_{2} \mathrm{O}\right){ }^{4+} & & \\ 4 \mathrm{1} & & & \\ \mathrm{TH} & -0.001918 & 0.001001 & -0.001038 \\ \mathrm{O} & -0.084547 & 1.548020 & 1.948048 \\ \mathrm{H} & 0.387862 & 2.394298 & 2.036490 \\ \mathrm{O} & -0.052502 & 0.892026 & -2.326091 \\ \mathrm{H} & 0.503099 & 0.601484 & -3.070398 \\ \mathrm{O} & 0.149360 & -2.454269 & 0.392639 \\ \mathrm{H} & 0.680316 & -2.885583 & 1.084814 \\ \mathrm{H} & -0.373498 & -3.145572 & -0.049737 \\ \mathrm{H} & -0.599410 & 1.411688 & 2.762568 \\ \mathrm{H} & -0.599144 & 1.633175 & -2.640782 \\ \mathrm{O} & 1.829993 & -0.997865 & -1.343396 \\ \mathrm{H} & 2.566522 & -0.531739 & -1.776522 \\ \mathrm{O} & 1.827001 & -0.529554 & 1.593219 \\ \mathrm{H} & 1.923400 & -0.135843 & 2.477732 \\ \mathrm{O} & 1.678430 & 1.808061 & -0.259098 \\ \mathrm{H} & 2.438338 & 1.984125 & 0.323145 \\ \mathrm{H} & 1.987985 & -1.953270 & -1.438988 \\ \mathrm{H} & 1.772434 & 2.375138 & -1.044237 \\ \mathrm{H} & 2.643965 & -1.021213 & 1.398163 \\ \mathrm{O} & -1.715930 & -1.194392 & -1.339443 \\ \mathrm{H} & -2.467631 & -1.712818 & -1.002536 \\ \mathrm{O} & -1.858541 & 1.629610 & -0.282062 \\ \mathrm{H} & -2.648811 & 1.512884 & -0.838527 \\ \mathrm{O} & -1.749354 & -0.708073 & 1.614763 \\ \mathrm{H} & -2.557658 & -0.219430 & 1.849608 \\ \mathrm{H} & -1.817024 & -1.595361 & 2.008520 \\ \mathrm{H} & -2.037596 & 2.365449 & 0.329182 \\ \mathrm{H} & -1.821853 & -1.116063 & -2.303726 \\ & & & \\ \mathrm{Th}\left(\mathrm{H}_{2} \mathrm{O}\right) 8 \mathrm{Cl}^{3+} & & \\ 31 & & & \\ \mathrm{CL} & 2.671773 & 0.031630 & -0.017885 \\ \mathrm{H} & 1.380838 & 2.603724 & -1.045136 \\ \mathrm{H} & -0.006643 & 2.629854 & -1.767348 \\ \mathrm{H} & -0.742594 & -0.034423 & -3.079517 \\ \mathrm{H} & -1.921575 & 0.749816 & -2.414708 \\ \mathrm{H} & 0.090822 & -2.400697 & -2.050715 \\ \mathrm{H} & 1.568807 & -2.139431 & -1.612898 \\ \mathrm{H} & -0.807053 & -0.533893 & 3.011517 \\ \mathrm{H} & -2.039304 & 0.254935 & 2.461232 \\ \mathrm{H} & -2.368571 & -2.055923 & 0.596723\end{array}$




$\begin{array}{lccc}\mathrm{H} & -2.360055 & -1.939245 & -0.969776 \\ \mathrm{H} & 1.551461 & -2.377992 & 1.269955 \\ \mathrm{H} & 0.068660 & -2.690984 & 1.654635 \\ \mathrm{H} & 1.369814 & 2.323110 & 1.592997 \\ \mathrm{H} & -0.001653 & 2.139151 & 2.320326 \\ \mathrm{O} & 0.612717 & 2.056590 & -1.283234 \\ \mathrm{O} & -1.213078 & 0.106093 & -2.239317 \\ \mathrm{O} & 0.686111 & -1.733883 & -1.664949 \\ \mathrm{O} & -1.282854 & -0.296604 & 2.196408 \\ \mathrm{O} & -2.029658 & -1.532603 & -0.150072 \\ \mathrm{O} & 0.671307 & -1.977948 & 1.378004 \\ \mathrm{O} & 0.623685 & 1.709897 & 1.710922 \\ \mathrm{TH} & -0.051368 & -0.000085 & -0.000892 \\ \mathrm{O} & -2.023743 & 1.561464 & 0.053570 \\ \mathrm{H} & -2.956078 & 1.291281 & 0.125140 \\ \mathrm{H} & -1.979770 & 2.506577 & 0.281222\end{array}$

$\begin{array}{cccc}\mathrm{Th}\left(\mathrm{H}_{2} \mathrm{O}\right){ }_{7} \mathrm{Cl}_{2}{ }^{2+}(\mathbf{g}) & & \\ 2 \mathrm{l} & & & \\ \mathrm{CL} & -2.519402 & -0.855626 & 0.015081 \\ \mathrm{CL} & 2.443135 & -0.965258 & 0.009387 \\ \mathrm{H} & -0.988005 & -2.444595 & -1.745153 \\ \mathrm{H} & 0.578986 & -2.518030 & -1.880599 \\ \mathrm{H} & 0.675462 & 0.697872 & -3.130481 \\ \mathrm{H} & 2.036705 & 0.350577 & -2.436944 \\ \mathrm{H} & -1.365245 & 2.397507 & -1.960272 \\ \mathrm{H} & -2.377660 & 1.230711 & -1.709413 \\ \mathrm{H} & 0.679085 & 0.780475 & 3.110654 \\ \mathrm{H} & 2.037760 & 0.400778 & 2.428736 \\ \mathrm{H} & 1.515740 & 2.763130 & 0.751944 \\ \mathrm{H} & 1.506985 & 2.758424 & -0.809839 \\ \mathrm{H} & -2.371525 & 1.274445 & 1.686868 \\ \mathrm{H} & -1.361612 & 2.452658 & 1.889184 \\ \mathrm{H} & -0.981481 & -2.404118 & 1.803434 \\ \mathrm{H} & 0.586262 & -2.476546 & 1.931396 \\ \mathrm{O} & -0.160594 & -1.954163 & -1.594812 \\ \mathrm{O} & 1.131752 & 0.671761 & -2.271781 \\ \mathrm{O} & -1.440449 & 1.486289 & -1.630629 \\ \mathrm{O} & 1.135727 & 0.724830 & 2.253444 \\ \mathrm{O} & 1.068545 & 2.386581 & -0.025360 \\ \mathrm{O} & -1.435239 & 1.529101 & 1.595482 \\ \mathrm{O} & -0.154349 & -1.918291 & 1.637421 \\ \mathrm{TH} & -0.000423 & 0.025365 & -0.000618\end{array}$

$\operatorname{Th}\left(\mathrm{H}_{2} \mathrm{O}\right)_{6} \mathrm{Cl}_{3}{ }^{1+}(\mathbf{f})$

11 


$\begin{array}{lrrr}\text { CL } & 1.067339 & -2.013068 & 1.415511 \\ \text { CL } & 0.967064 & 1.765724 & 1.764479 \\ \text { CL } & 2.509609 & 0.131268 & -1.062890 \\ \text { H } & -1.663836 & -2.758126 & -0.475083 \\ \text { H } & -2.347444 & -1.991930 & 0.697383 \\ \text { H } & -2.012504 & 0.365063 & 2.446041 \\ \text { H } & -1.085394 & -0.827076 & 2.842168 \\ \text { H } & -1.727940 & 2.583190 & 0.922652 \\ \mathrm{H} & -2.559324 & 1.968853 & -0.259818 \\ \mathrm{H} & -2.552576 & -0.460427 & -2.017118 \\ \mathrm{H} & -1.766966 & 0.714090 & -2.690172 \\ \mathrm{H} & -0.426045 & -1.897039 & -2.641266 \\ \mathrm{H} & 1.103190 & -1.708416 & -2.380193 \\ \mathrm{H} & 1.096901 & 2.227532 & -1.880857 \\ \mathrm{H} & -0.286364 & 2.848435 & -1.496523 \\ \mathrm{O} & -1.886060 & -1.868542 & -0.150746 \\ \mathrm{O} & -1.513370 & -0.388431 & 2.086940 \\ \mathrm{O} & -1.900141 & 1.765661 & 0.425503 \\ \mathrm{O} & -1.949770 & 0.281741 & -1.838610 \\ \mathrm{O} & 0.229254 & -1.688706 & -1.953532 \\ \mathrm{O} & 0.183509 & 2.007132 & -1.628127 \\ \mathrm{TH} & -0.092525 & 0.000426 & -0.050993\end{array}$

$\mathrm{Th}\left(\mathrm{H}_{2} \mathrm{O}\right)_{5} \mathrm{Cl}_{4}(\mathbf{e})$

01

$\begin{array}{lccc}\text { CL } & -0.564976 & 2.564158 & -0.867690 \\ \text { CL } & -2.581068 & -0.620113 & -0.832159 \\ \text { CL } & 2.637078 & 0.632172 & -0.606346 \\ \text { CL } & 0.634674 & -2.553943 & -0.852129 \\ \text { H } & -2.230130 & 0.587182 & 2.228851 \\ \text { H } & -2.160034 & 1.915110 & 1.412244 \\ \mathrm{H} & 2.010496 & -1.951702 & 1.530964 \\ \mathrm{H} & 2.043077 & -0.642572 & 2.380524 \\ \mathrm{H} & 0.682247 & -0.673643 & -2.907012 \\ \mathrm{H} & 0.372778 & 0.854830 & -2.926140 \\ \mathrm{H} & -0.641625 & -2.122723 & 2.303600 \\ \mathrm{H} & 0.424080 & 2.081830 & 2.374986 \\ \mathrm{H} & -1.975934 & -2.034399 & 1.488538 \\ \mathrm{H} & 1.827461 & 2.008456 & 1.682924 \\ \mathrm{O} & -1.622477 & 1.182011 & 1.757045 \\ \mathrm{O} & -1.211537 & -1.518897 & 1.797332 \\ \mathrm{O} & 1.039689 & 1.487188 & 1.913110 \\ \mathrm{O} & 1.460988 & -1.218145 & 1.855688 \\ \mathrm{O} & 0.114750 & 0.012122 & -2.515159 \\ \mathrm{TH} & -0.008231 & 0.000497 & 0.062866\end{array}$




$\begin{array}{cccc}\mathrm{Th}\left(\mathrm{H}_{2} \mathrm{O}\right)_{4} \mathrm{Cl}_{4}(\mathbf{b}) & & \\ 01 & & & \\ \mathrm{CL} & -1.661044 & 1.922895 & -0.967495 \\ \mathrm{CL} & -2.062188 & -1.764503 & 0.560074 \\ \mathrm{CL} & 2.077357 & -1.743742 & -0.568049 \\ \mathrm{CL} & 1.645227 & 1.929939 & 0.978325 \\ \mathrm{H} & -1.685639 & -1.437750 & -2.281239 \\ \mathrm{H} & -0.549989 & -0.649798 & -3.017312 \\ \mathrm{H} & 1.006434 & 1.815968 & -2.345651 \\ \mathrm{H} & 2.102450 & 0.714799 & -2.230148 \\ \mathrm{H} & 1.699661 & -1.435092 & 2.271366 \\ \mathrm{H} & 0.559877 & -0.659795 & 3.013955 \\ \mathrm{H} & -2.108777 & 0.673578 & 2.235378 \\ \mathrm{H} & -1.033717 & 1.795722 & 2.349509 \\ \mathrm{O} & -0.778607 & -1.101993 & -2.187225 \\ \mathrm{O} & 1.149832 & 0.879999 & -2.122690 \\ \mathrm{O} & 0.789826 & -1.105928 & 2.180980 \\ \mathrm{O} & -1.159768 & 0.857386 & 2.126141 \\ \mathrm{TH} & 0.000116 & -0.032348 & -0.000245 \\ & & & \\ \mathrm{Th}\left(\mathrm{H}_{2} \mathrm{O}\right)_{4} \mathrm{Cl}_{4}(\mathbf{c}) & & \\ 0 \mathrm{l} & & & \\ \mathrm{CL} & 1.934868 & 0.112633 & -1.906530 \\ \mathrm{CL} & -1.934868 & -0.112633 & -1.906530 \\ \mathrm{CL} & -1.686803 & 1.486235 & 1.635976 \\ \mathrm{CL} & 1.686803 & -1.486235 & 1.635976 \\ \mathrm{H} & 0.352130 & 2.528958 & -1.792142 \\ \mathrm{H} & -0.352130 & -2.528958 & -1.792142 \\ \mathrm{H} & -0.823437 & 2.914640 & -0.843161 \\ \mathrm{H} & 0.823437 & -2.914640 & -0.843161 \\ \mathrm{H} & 2.063670 & 1.328769 & 2.035894 \\ \mathrm{H} & -2.063670 & -1.328769 & 2.035894 \\ \mathrm{H} & 1.452174 & 2.525599 & 1.230832 \\ \mathrm{H} & -1.452174 & -2.525599 & 1.230832 \\ \mathrm{O} & 0.000000 & 2.399429 & -0.894604 \\ \mathrm{O} & 0.000000 & -2.399429 & -0.894604 \\ \mathrm{O} & 1.631625 & 1.570498 & 1.199663 \\ \mathrm{O} & -1.631625 & -1.570498 & 1.199663 \\ \mathrm{TH} & 0.000000 & 0.000000 & 0.033945 \\ & & & \\ \mathrm{Th}\left(\mathrm{H}_{2} \mathrm{O}\right)_{4} \mathrm{Cl} l_{4}(\mathbf{d}) & & \\ 01 & & & \\ \mathrm{CL} & -2.697532 & 0.604776 & 0.086978 \\ \mathrm{CL} & -0.712276 & -2.331002 & -1.207615 \\ \mathrm{CL} & 2.530484 & -0.134395 & -1.009870 \\ \mathrm{CL} & 0.264529 & 2.728541 & -0.166724\end{array}$




$\begin{array}{cccc}\mathrm{H} & -0.957126 & -0.021768 & -2.926936 \\ \mathrm{H} & -0.673867 & 1.484346 & -2.621617 \\ \mathrm{H} & 1.783004 & 0.298823 & 2.651320 \\ \mathrm{H} & 1.601960 & 1.760910 & 2.116254 \\ \mathrm{H} & -0.656648 & -1.135191 & 3.007376 \\ \mathrm{H} & 2.207350 & -2.049967 & 0.954968 \\ \mathrm{H} & -2.020265 & -0.736556 & 2.350637 \\ \mathrm{H} & 0.919893 & -2.666004 & 1.574926 \\ \mathrm{O} & -0.419070 & 0.577192 & -2.381831 \\ \mathrm{O} & 1.205853 & 0.873112 & 2.119127 \\ \mathrm{O} & -1.097550 & -0.973203 & 2.156057 \\ \mathrm{O} & 1.347639 & -1.822067 & 1.349224 \\ \mathrm{TH} & -0.000531 & -0.010328 & 0.066727 \\ & & & \\ \mathrm{Th}(\mathrm{H} 2 \mathrm{O})_{3} \mathrm{Cl}{ }_{5}^{1-} & & \\ -1 \mathrm{1} & & & \\ \mathrm{CL} & -1.988874 & 1.706643 & -0.897191 \\ \mathrm{CL} & -1.091757 & -1.746348 & -1.807702 \\ \mathrm{CL} & 1.098432 & 2.448560 & 0.803951 \\ \mathrm{CL} & -2.036049 & -0.601785 & 1.792861 \\ \mathrm{TH} & 0.019629 & -0.001252 & 0.027070 \\ \mathrm{CL} & 2.546220 & -0.909349 & -0.708646 \\ \mathrm{O} & 0.391445 & -2.405719 & 1.010127 \\ \mathrm{O} & 1.186266 & -0.146160 & 2.319055 \\ \mathrm{H} & 0.967899 & -0.870621 & 2.929166 \\ \mathrm{H} & 0.944852 & -3.002320 & 0.478128 \\ \mathrm{H} & 1.290121 & 0.662270 & 2.847947 \\ \mathrm{H} & -0.423152 & -2.896036 & 1.213478 \\ \mathrm{O} & 0.732402 & 1.043307 & -2.224586 \\ \mathrm{H} & 1.685227 & 1.140257 & -2.391910 \\ \mathrm{H} & 0.312010 & 1.886443 & -2.465507\end{array}$

$\mathrm{Th}\left(\mathrm{H}_{2} \mathrm{O}\right)_{2} \mathrm{Cl}_{6}{ }^{2-}$ (a)

$-21$

$\begin{array}{lccc}\text { CL } & 1.619869 & 0.590978 & 2.213507 \\ \text { CL } & -0.770364 & 2.654940 & 0.434545 \\ \text { CL } & 2.009484 & 1.393040 & -1.341199 \\ \text { CL } & 1.844630 & -2.016226 & -0.710215 \\ \text { CL } & -2.661535 & -0.196451 & 0.995659 \\ \text { CL } & -1.211048 & 0.257048 & -2.463437 \\ \text { H } & -0.692380 & -2.851176 & -1.285645 \\ \text { H } & 0.717179 & -2.076849 & 2.321026 \\ \text { O } & -0.130351 & -1.857161 & 1.899751 \\ \text { O } & -1.165183 & -2.361659 & -0.590922 \\ \text { TH } & -0.013542 & -0.021807 & 0.029571 \\ \text { H } & -2.090571 & -2.291246 & -0.880167\end{array}$


$\mathrm{H} \quad-0.478770 \quad-2.684092 \quad 1.522137$

Table S14. Cartesian coordinates in $\AA$ for optimized geometries for gas phase organic bases (neutral and protonated) at the B3LYP/DZVP2 level.

\begin{tabular}{|c|c|c|c|}
\hline \multicolumn{4}{|c|}{ Quino $(\mathrm{OH})$} \\
\hline \\
\hline $\mathrm{C}$ & -2.749597 & -0.395162 & 0.000088 \\
\hline $\mathrm{C}$ & -1.710010 & -1.320091 & -0.111466 \\
\hline $\mathrm{C}$ & -0.373304 & -0.880744 & -0.098305 \\
\hline $\mathrm{C}$ & -0.101468 & 0.502751 & 0.011812 \\
\hline $\mathrm{C}$ & -1.158778 & 1.418430 & 0.118562 \\
\hline $\mathrm{C}$ & -2.482627 & 0.979655 & 0.120280 \\
\hline $\mathrm{H}$ & -3.776923 & -0.748465 & -0.004726 \\
\hline $\mathrm{H}$ & -1.930252 & -2.380221 & -0.206993 \\
\hline $\mathrm{C}$ & 1.302998 & 0.997121 & -0.063586 \\
\hline $\mathrm{H}$ & -0.908865 & 2.472124 & 0.186606 \\
\hline $\mathrm{H}$ & -3.298150 & 1.690386 & 0.204350 \\
\hline $\mathrm{C}$ & 1.950193 & -1.348465 & 0.360136 \\
\hline $\mathrm{H}$ & 1.887802 & -1.370748 & 1.464275 \\
\hline $\mathrm{N}$ & 2.254948 & -0.006142 & -0.114662 \\
\hline $\mathrm{N}$ & 0.695719 & -1.773402 & -0.250679 \\
\hline $\mathrm{H}$ & 0.457181 & -2.737094 & -0.050790 \\
\hline $\mathrm{H}$ & 3.211685 & 0.318461 & -0.050780 \\
\hline $\mathrm{H}$ & 2.748207 & -2.025539 & 0.043716 \\
\hline $\mathrm{O}$ & 1.611274 & 2.189619 & -0.131675 \\
\hline
\end{tabular}

Quino (=O)

01

$\begin{array}{rrrc}\mathrm{C} & -2.757203 & 0.379497 & 0.107466 \\ \mathrm{C} & -1.712831 & 1.292361 & 0.244291 \\ \mathrm{C} & -0.374163 & 0.878744 & 0.088437 \\ \mathrm{C} & -0.107261 & -0.478091 & -0.191081 \\ \mathrm{C} & -1.162245 & -1.389453 & -0.328226 \\ \mathrm{C} & -2.486118 & -0.968730 & -0.184689 \\ \mathrm{H} & -3.784547 & 0.712139 & 0.225981 \\ \mathrm{H} & -1.901353 & 2.337993 & 0.465177 \\ \mathrm{C} & 1.323357 & -0.948598 & -0.283763 \\ \mathrm{H} & -0.943084 & -2.434400 & -0.532126 \\ \mathrm{H} & -3.299769 & -1.679544 & -0.291462 \\ \mathrm{C} & 1.827825 & 1.460840 & -0.116198 \\ \mathrm{H} & 3.158500 & 0.019743 & -0.693096 \\ \mathrm{H} & 2.644337 & 2.182058 & -0.093362 \\ \mathrm{H} & 1.446078 & -1.664002 & -1.106488 \\ \mathrm{~N} & 2.174713 & 0.198626 & -0.543799 \\ \mathrm{~N} & 0.638262 & 1.847347 & 0.201263 \\ \mathrm{O} & 1.661684 & -1.615089 & 0.952323 \\ \mathrm{H} & 2.387382 & -2.234505 & 0.787126\end{array}$




$$
\begin{aligned}
& \text { QuinoH }{ }^{+} \\
& 11 \\
& \begin{array}{llll}
\text { C } & -2.774642 & 0.390286 & 0.108854
\end{array} \\
& \begin{array}{llll}
\text { C } & -1.721892 & 1.296972 & 0.237871
\end{array} \\
& \begin{array}{llll}
\text { C } & -0.409851 & 0.836616 & 0.075289
\end{array} \\
& \begin{array}{llll}
\text { C } & -0.128822 & -0.509031 & -0.199346
\end{array} \\
& \begin{array}{llll}
\text { C } & -1.197941 & -1.402310 & -0.332361
\end{array} \\
& \begin{array}{llll}
\text { C } & -2.514045 & -0.957663 & -0.182994
\end{array} \\
& \begin{array}{llll}
\mathrm{H} & 0.492678 & 2.716706 & 0.386925
\end{array} \\
& \begin{array}{llll}
\mathrm{H} & -3.796496 & 0.733817 & 0.230841
\end{array} \\
& \mathrm{H} \quad-1.919404 \quad 2.343356 \quad 0.453364 \\
& \begin{array}{llll}
\text { C } & 1.298204 & -1.008392 & -0.269632
\end{array} \\
& \mathrm{H} \quad-1.000322-2.450550-0.535310 \\
& \mathrm{H} \quad-3.336030 \quad-1.658107 \quad-0.286120 \\
& \text { C } \quad 1.910824 \quad 1.392253 \quad-0.152782 \\
& \mathrm{H} \quad 3.183015 \quad-0.059334 \quad-0.686251 \\
& \text { H } 2.691518 \quad 2.145698 \quad-0.149736 \\
& \text { H } \quad 1.432071 \quad-1.684600 \quad-1.119365 \\
& \begin{array}{llll}
\mathrm{N} & 2.209658 & 0.146414 & -0.489338
\end{array} \\
& \mathrm{~N} \quad 0.676165 \quad 1.747190 \quad 0.152895 \\
& \begin{array}{llll}
\mathrm{O} & 1.639468 & -1.641830 & 0.945723
\end{array} \\
& \begin{array}{llll}
\mathrm{H} & 2.165448 & -2.439969 & 0.785569
\end{array} \\
& \text { 2-MePy } \\
& 01 \\
& \begin{array}{llll}
\text { C } & -1.876546 & -0.070873 & 0.000155
\end{array} \\
& \begin{array}{llll}
\text { C } & -1.074016 & -1.217799 & 0.000080
\end{array} \\
& \begin{array}{llll}
\text { C } & 0.879482 & 0.006350 & -0.000295
\end{array} \\
& \begin{array}{llll}
\text { C } & 0.158515 & 1.212203 & -0.000156
\end{array} \\
& \begin{array}{llll}
\mathrm{C} & -1.239695 & 1.174452 & 0.000055
\end{array} \\
& \begin{array}{llll}
\mathrm{H} & -2.958595 & -0.156002 & 0.000238
\end{array} \\
& \begin{array}{llll}
\mathrm{H} & -1.532740 & -2.205454 & -0.000025
\end{array} \\
& \begin{array}{lrrr}
\mathrm{H} & 0.686479 & 2.160778 & -0.000269
\end{array} \\
& \begin{array}{llll}
\mathrm{H} & -1.817266 & 2.094629 & 0.000096
\end{array} \\
& \begin{array}{llll}
\mathrm{N} & 0.265640 & -1.192700 & -0.000217
\end{array} \\
& \begin{array}{llll}
\text { C } & 2.391157 & -0.012316 & 0.000164
\end{array} \\
& \begin{array}{llll}
\mathrm{H} & 2.760289 & -0.542605 & 0.883657
\end{array} \\
& \text { H } \quad 2.760686 \quad-0.552313-0.877162 \\
& \begin{array}{llll}
\mathrm{H} & 2.808282 & 0.997760 & -0.005031
\end{array} \\
& \text { 2-MePyH }{ }^{+} \\
& 11 \\
& \begin{array}{llll}
\text { C } & -1.902523 & -0.053599 & 0.000229
\end{array} \\
& \begin{array}{llll}
\text { C } & -1.133382 & -1.203080 & 0.000003
\end{array} \\
& \begin{array}{llll}
\text { C } & 0.903664 & 0.075839 & -0.000309
\end{array}
\end{aligned}
$$




$\begin{array}{rrrr}\mathrm{C} & 0.149411 & 1.250516 & -0.000252 \\ \mathrm{C} & -1.248228 & 1.189643 & 0.000066 \\ \mathrm{H} & -2.983667 & -0.127863 & 0.000408 \\ \mathrm{H} & -1.547185 & -2.204712 & -0.000011 \\ \mathrm{H} & 0.761637 & -1.962067 & -0.000344 \\ \mathrm{H} & 0.662920 & 2.204960 & -0.000439 \\ \mathrm{H} & -1.828975 & 2.107169 & 0.000122 \\ \mathrm{~N} & 0.219218 & -1.100513 & -0.000196 \\ \mathrm{C} & 2.403258 & 0.019414 & 0.000240 \\ \mathrm{H} & 2.772813 & -0.498827 & 0.891699 \\ \mathrm{H} & 2.773609 & -0.513096 & -0.882362 \\ \mathrm{H} & 2.821126 & 1.025627 & -0.007560\end{array}$

Phen

01

$\begin{array}{llll}\text { C } & 0.000000 & 3.488238 & -0.373575\end{array}$

$\begin{array}{llll}\text { C } & 0.000000 & 2.835252 & 0.845358\end{array}$

$\begin{array}{llll}\text { C } & 0.000000 & 1.419169 & 0.870302\end{array}$

$\begin{array}{llll}\text { C } & 0.000000 & 0.730029 & -0.377887\end{array}$

$\begin{array}{llll}\text { C } & 0.000000 & 2.705322 & -1.552214\end{array}$

$\begin{array}{llll}\text { C } & 0.000000 & 0.682284 & 2.106067\end{array}$

$\begin{array}{llll}\text { C } & 0.000000 & -0.730029 & -0.377887\end{array}$

$\begin{array}{llll}\text { C } & 0.000000 & -1.419169 & 0.870302\end{array}$

$\begin{array}{llll}\text { C } & 0.000000 & -0.682284 & 2.106067\end{array}$

$\begin{array}{llll}\text { C } & 0.000000 & -2.835252 & 0.845358\end{array}$

$\begin{array}{llll}\mathrm{H} & 0.000000 & -3.389773 & 1.779912\end{array}$

C $\quad 0.000000 \quad-3.488238-0.373575$

$\begin{array}{llll}\text { C } & 0.000000 & -2.705322 & -1.552214\end{array}$

$\begin{array}{llll}\mathrm{H} & 0.000000 & 1.236040 & 3.041412\end{array}$

$\mathrm{H} \quad 0.000000 \quad 4.571738 \quad-0.438865$

$\mathrm{H} \quad 0.000000 \quad 3.389773 \quad 1.779912$

$\mathrm{H} \quad 0.000000 \quad 3.197057 \quad-2.524516$

$\mathrm{H} \quad 0.000000 \quad-1.236040 \quad 3.041412$

$\mathrm{H} \quad 0.000000 \quad-4.571738-0.438865$

$\mathrm{H} \quad 0.000000 \quad-3.197057 \quad-2.524516$

$\mathrm{N} \quad 0.000000 \quad-1.381247 \quad-1.566608$

$\mathrm{N} \quad 0.000000 \quad 1.381247 \quad-1.566608$

PhenH $^{+}$

11
$\begin{array}{llll}\text { C } & -0.980652 & 3.344662 & 0.000000\end{array}$
$\begin{array}{llll}\text { C } & -1.873030 & 2.274360 & 0.000000\end{array}$
$\begin{array}{llll}\text { C } & -1.401091 & 0.939993 & 0.000000\end{array}$
$\begin{array}{llll}\text { C } & 0.000000 & 0.744358 & 0.000000\end{array}$
$\begin{array}{llll}\text { C } & 0.393964 & 3.091252 & 0.000000\end{array}$
$\begin{array}{llll}\text { C } & -2.249877 & -0.221704 & 0.000000\end{array}$ 


$$
\begin{array}{cccc}
\mathrm{C} & 0.586456 & -0.565307 & 0.000000 \\
\mathrm{C} & -0.281587 & -1.684397 & 0.000000 \\
\mathrm{C} & -1.707764 & -1.478425 & 0.000000 \\
\mathrm{C} & 0.332915 & -2.963077 & 0.000000 \\
\mathrm{H} & -0.276707 & -3.861677 & 0.000000 \\
\mathrm{C} & 1.714456 & -3.040227 & 0.000000 \\
\mathrm{C} & 2.480801 & -1.845938 & 0.000000 \\
\mathrm{H} & -3.326220 & -0.082585 & 0.000000 \\
\mathrm{H} & -1.330178 & 4.370356 & 0.000000 \\
\mathrm{H} & -2.942741 & 2.463230 & 0.000000 \\
\mathrm{H} & 1.828435 & 1.589140 & 0.000000 \\
\mathrm{H} & 1.143686 & 3.873629 & 0.000000 \\
\mathrm{H} & -2.356933 & -2.349310 & 0.000000 \\
\mathrm{H} & 2.222261 & -3.999014 & 0.000000 \\
\mathrm{H} & 3.566821 & -1.896291 & 0.000000 \\
\mathrm{~N} & 1.938871 & -0.635951 & 0.000000 \\
\mathrm{~N} & 0.830276 & 1.824412 & 0.000000
\end{array}
$$

$$
\begin{aligned}
& \text { 4-ClPy } \\
& 01 \\
& \begin{array}{llll}
\text { C } & 0.000000 & 1.207379 & -0.198357
\end{array} \\
& \text { C } \quad 0.000000 \quad 1.143205 \quad-1.597505 \\
& \text { C } \quad 0.000000 \quad-1.143205 \quad-1.597505 \\
& \begin{array}{llll}
\text { C } & 0.000000 & -1.207379 & -0.198357
\end{array} \\
& \begin{array}{llll}
\text { C } & 0.000000 & 0.000000 & 0.504248
\end{array} \\
& \mathrm{H} \quad 0.000000 \quad 2.160820 \quad 0.317511 \\
& \mathrm{H} \quad 0.000000 \quad 2.064883 \quad-2.176500 \\
& \mathrm{H} \quad 0.000000 \quad-2.064883-2.176500 \\
& \mathrm{H} \quad 0.000000 \quad-2.160820 \quad 0.317511 \\
& \begin{array}{llll}
\mathrm{N} & 0.000000 & 0.000000 & -2.299461
\end{array} \\
& \begin{array}{llll}
\text { CL } & 0.000000 & 0.000000 & 2.255239
\end{array}
\end{aligned}
$$

$$
\begin{array}{cccc}
\text { 4-ClPyH } \\
11 & & & \\
\mathrm{C} & 0.000000 & 1.219817 & -0.144334 \\
\mathrm{C} & 0.000000 & 1.190117 & -1.526698 \\
\mathrm{C} & 0.000000 & -1.190117 & -1.526698 \\
\mathrm{C} & 0.000000 & -1.219817 & -0.144334 \\
\mathrm{C} & 0.000000 & 0.000000 & 0.561288 \\
\mathrm{H} & 0.000000 & 2.169805 & 0.377320 \\
\mathrm{H} & 0.000000 & 2.086166 & -2.136708 \\
\mathrm{H} & 0.000000 & 0.000000 & -3.194014 \\
\mathrm{H} & 0.000000 & -2.086166 & -2.136708 \\
\mathrm{H} & 0.000000 & -2.169805 & 0.377320 \\
\mathrm{~N} & 0.000000 & 0.000000 & -2.176695 \\
\mathrm{CL} & 0.000000 & 0.000000 & 2.272607
\end{array}
$$




$$
\begin{array}{rrrc}
4,4 & \text {-Bipy } \\
01 & & & \\
\text { C } & 0.000000 & 0.000000 & -0.742892 \\
\text { C } & -0.364372 & 1.139904 & -1.478806 \\
\text { C } & -0.350392 & 1.086895 & -2.876214 \\
\text { C } & 0.350392 & -1.086895 & -2.876214 \\
\text { C } & 0.364372 & -1.139904 & -1.478806 \\
\text { C } & 0.000000 & 0.000000 & 0.742892 \\
\text { C } & 0.364372 & 1.139904 & 1.478806 \\
\text { C } & 0.350392 & 1.086895 & 2.876214 \\
\text { C } & -0.350392 & -1.086895 & 2.876214 \\
\text { C } & -0.364372 & -1.139904 & 1.478806 \\
\text { H } & -0.680935 & 2.049242 & -0.978278 \\
\text { H } & -0.639771 & 1.961959 & -3.455265 \\
\text { H } & 0.639771 & -1.961959 & -3.455265 \\
\text { H } & 0.680935 & -2.049242 & -0.978278 \\
\text { H } & 0.680935 & 2.049242 & 0.978278 \\
\text { H } & 0.639771 & 1.961959 & 3.455265 \\
\text { H } & -0.639771 & -1.961959 & 3.455265 \\
\text { H } & -0.680935 & -2.049242 & 0.978278 \\
\text { N } & 0.000000 & 0.000000 & 3.581365 \\
\text { N } & 0.000000 & 0.000000 & -3.581365
\end{array}
$$

$\begin{array}{rrrr}\text { 4,4'-BipyH }{ }^{+} & & \\ 11 & & & \\ \mathrm{C} & 0.000000 & 0.000000 & 0.788640 \\ \mathrm{C} & 0.604650 & 1.041224 & 1.516547 \\ \mathrm{C} & 0.584164 & 0.986561 & 2.914223 \\ \mathrm{C} & -0.584164 & -0.986561 & 2.914223 \\ \mathrm{C} & -0.604650 & -1.041224 & 1.516547 \\ \mathrm{C} & 0.000000 & 0.000000 & -0.686513 \\ \mathrm{C} & 0.001346 & 1.208755 & -1.423186 \\ \mathrm{C} & 0.000000 & 1.187575 & -2.803981 \\ \mathrm{C} & 0.000000 & -1.187575 & -2.803981 \\ \mathrm{C} & -0.001346 & -1.208755 & -1.423186 \\ \mathrm{H} & 1.119555 & 1.859881 & 1.024596 \\ \mathrm{H} & 1.059914 & 1.771666 & 3.497353 \\ \mathrm{H} & -1.059914 & -1.771666 & 3.497353 \\ \mathrm{H} & -1.119555 & -1.859881 & 1.024596 \\ \mathrm{H} & -0.023803 & 2.167486 & -0.919709 \\ \mathrm{H} & -0.009540 & 2.083919 & -3.413334 \\ \mathrm{H} & 0.000000 & 0.000000 & -4.476637 \\ \mathrm{H} & 0.009540 & -2.083919 & -3.413334 \\ \mathrm{H} & 0.023803 & -2.167486 & -0.919709 \\ \mathrm{~N} & 0.000000 & 0.000000 & -3.459747\end{array}$


$\begin{array}{llll}\mathrm{N} & 0.000000 & 0.000000 & 3.608722\end{array}$

$\begin{array}{lccc}\text { 3-ClPy } \\ 01 \\ \text { C } & 0.000000 & -0.493566 & 0.000000 \\ \mathrm{C} & -1.186967 & 0.250324 & 0.000000 \\ \mathrm{C} & -0.015752 & 2.226449 & 0.000000 \\ \mathrm{C} & 1.217091 & 1.563742 & 0.000000 \\ \mathrm{C} & 1.231771 & 0.164894 & 0.000000 \\ \mathrm{H} & -2.149936 & -0.254672 & 0.000000 \\ \mathrm{H} & -0.056127 & 3.313718 & 0.000000 \\ \mathrm{H} & 2.144505 & 2.127923 & 0.000000 \\ \mathrm{~N} & -1.196650 & 1.589814 & 0.000000 \\ \mathrm{H} & 2.161049 & -0.394714 & 0.000000 \\ \mathrm{CL} & -0.070577 & -2.246589 & 0.000000\end{array}$

$\begin{array}{lccc}\text { 3-ClPyH } \\ 11 \\ \text { 1 } & & & \\ \mathrm{C} & 0.000000 & -0.552648 & 0.000000 \\ \mathrm{C} & -1.164782 & 0.212913 & 0.000000 \\ \mathrm{C} & 0.114987 & 2.227609 & 0.000000 \\ \mathrm{C} & 1.297277 & 1.498504 & 0.000000 \\ \mathrm{C} & 1.246204 & 0.100045 & 0.000000 \\ \mathrm{H} & -2.159496 & -0.216869 & 0.000000 \\ \mathrm{H} & 0.071833 & 3.310155 & 0.000000 \\ \mathrm{H} & 2.247483 & 2.020627 & 0.000000 \\ \mathrm{~N} & -1.062406 & 1.561856 & 0.000000 \\ \mathrm{H} & 2.160546 & -0.485410 & 0.000000 \\ \mathrm{CL} & -0.112961 & -2.269608 & 0.000000 \\ \mathrm{H} & -1.925302 & 2.103315 & 0.000000\end{array}$

Phthal

01

$\begin{array}{lrrr}\mathrm{C} & 0.000000 & 0.710613 & 2.409772 \\ \mathrm{C} & 0.000000 & 1.415760 & 1.218963 \\ \mathrm{C} & 0.000000 & 0.709606 & -0.012121 \\ \mathrm{C} & 0.000000 & -0.709606 & -0.012121 \\ \mathrm{C} & 0.000000 & -1.415760 & 1.218963 \\ \mathrm{C} & 0.000000 & -0.710613 & 2.409772 \\ \mathrm{H} & 0.000000 & 2.419367 & -1.374156 \\ \mathrm{H} & 0.000000 & 1.242380 & 3.356791 \\ \mathrm{H} & 0.000000 & 2.502131 & 1.217302 \\ \mathrm{C} & 0.000000 & 1.332861 & -1.296970 \\ \mathrm{C} & 0.000000 & -1.332861 & -1.296970 \\ \mathrm{H} & 0.000000 & -2.502131 & 1.217302 \\ \mathrm{H} & 0.000000 & -1.242380 & 3.356791\end{array}$




$$
\begin{array}{llrl}
\mathrm{H} & 0.000000 & -2.419367 & -1.374156 \\
\mathrm{~N} & 0.000000 & -0.685802 & -2.445401 \\
\mathrm{~N} & 0.000000 & 0.685802 & -2.445401
\end{array}
$$

\begin{tabular}{lrrr}
\multicolumn{4}{l}{ PhthalH ${ }^{+}$} \\
11 \\
C & 2.410521 & 0.821688 & 0.000000 \\
$\mathrm{C}$ & 1.193450 & 1.481228 & 0.000000 \\
$\mathrm{C}$ & 0.000000 & 0.714266 & 0.000000 \\
$\mathrm{C}$ & 0.056616 & -0.717994 & 0.000000 \\
$\mathrm{C}$ & 1.311465 & -1.364733 & 0.000000 \\
$\mathrm{C}$ & 2.468484 & -0.597276 & 0.000000 \\
$\mathrm{H}$ & -1.462512 & 2.363340 & 0.000000 \\
$\mathrm{H}$ & 3.334772 & 1.390707 & 0.000000 \\
$\mathrm{H}$ & 1.146956 & 2.565835 & 0.000000 \\
$\mathrm{C}$ & -1.287169 & 1.291823 & 0.000000 \\
$\mathrm{C}$ & -1.187986 & -1.424648 & 0.000000 \\
$\mathrm{H}$ & 1.364763 & -2.448892 & 0.000000 \\
$\mathrm{H}$ & 3.438183 & -1.085787 & 0.000000 \\
$\mathrm{H}$ & -1.212089 & -2.511335 & 0.000000 \\
$\mathrm{H}$ & -3.294281 & 0.908897 & 0.000000 \\
$\mathrm{~N}$ & -2.371082 & -0.850293 & 0.000000 \\
$\mathrm{~N}$ & -2.358643 & 0.506166 & 0.000000
\end{tabular}

$\begin{array}{crrr}\text { Terpy } & & & \\ 01 & & & \\ \text { C } & 0.000000 & 1.156888 & 0.784723 \\ \text { C } & 0.000000 & -1.156888 & 0.784723 \\ \text { C } & 0.000000 & -1.204169 & 2.188144 \\ \text { C } & 0.000000 & 0.000000 & 2.893968 \\ \text { C } & 0.000000 & 1.204169 & 2.188144 \\ \text { C } & 0.000000 & 2.419734 & -0.013465 \\ \text { C } & 0.000000 & 4.723066 & -0.009435 \\ \text { C } & 0.000000 & 4.790763 & -1.407861 \\ \text { C } & 0.000000 & 3.588114 & -2.124170 \\ \text { C } & 0.000000 & 2.384559 & -1.417700 \\ \text { H } & 0.000000 & -2.167685 & 2.681732 \\ \text { H } & 0.000000 & 0.000000 & 3.980608 \\ \text { H } & 0.000000 & 2.167685 & 2.681732 \\ \text { H } & 0.000000 & 5.636749 & 0.582389 \\ \text { H } & 0.000000 & 5.751984 & -1.911829 \\ \text { H } & 0.000000 & 3.590797 & -3.210588 \\ \text { H } & 0.000000 & 1.425042 & -1.919220 \\ \text { C } & 0.000000 & -2.419734 & -0.013465 \\ \text { C } & 0.000000 & -2.384559 & -1.417700 \\ \text { C } & 0.000000 & -3.588114 & -2.124170\end{array}$




$\begin{array}{llll}\mathrm{H} & 0.000000 & -1.425042 & -1.919220 \\ \mathrm{C} & 0.000000 & -4.723066 & -0.009435 \\ \mathrm{C} & 0.000000 & -4.790763 & -1.407861 \\ \mathrm{H} & 0.000000 & -3.590797 & -3.210588 \\ \mathrm{H} & 0.000000 & -5.636749 & 0.582389 \\ \mathrm{H} & 0.000000 & -5.751984 & -1.911829 \\ \mathrm{~N} & 0.000000 & 0.000000 & 0.102019 \\ \mathrm{~N} & 0.000000 & 3.576192 & 0.678119 \\ \mathrm{~N} & 0.000000 & -3.576192 & 0.678119\end{array}$

\begin{tabular}{crrr}
\multicolumn{5}{c}{ TerpyH ${ }^{+}($side $)$} & & \\
11 & & & \\
$\mathrm{C}$ & -1.145044 & 0.913684 & 0.000010 \\
$\mathrm{C}$ & 1.172596 & 1.019583 & 0.000002 \\
$\mathrm{C}$ & 1.200845 & 2.414780 & 0.000034 \\
$\mathrm{C}$ & -0.037351 & 3.071617 & 0.000054 \\
$\mathrm{C}$ & -1.213813 & 2.324339 & 0.000043 \\
$\mathrm{C}$ & -2.349536 & 0.027747 & 0.000007 \\
$\mathrm{C}$ & -3.141466 & -2.132033 & 0.000105 \\
$\mathrm{C}$ & -4.478818 & -1.709406 & -0.000004 \\
$\mathrm{C}$ & -4.735575 & -0.337339 & -0.000117 \\
$\mathrm{C}$ & -3.652505 & 0.547466 & -0.000109 \\
$\mathrm{H}$ & 2.127128 & 2.977431 & 0.000053 \\
$\mathrm{H}$ & -0.079874 & 4.156192 & 0.000086 \\
$\mathrm{H}$ & -2.168169 & 2.835574 & 0.000068 \\
$\mathrm{H}$ & -2.907157 & -3.194512 & 0.000203 \\
$\mathrm{H}$ & -5.284275 & -2.436260 & -0.000003 \\
$\mathrm{H}$ & -5.753628 & 0.039196 & -0.000211 \\
$\mathrm{H}$ & -3.837921 & 1.614943 & -0.000202 \\
$\mathrm{C}$ & 2.380924 & 0.157983 & -0.000014 \\
$\mathrm{C}$ & 3.721604 & 0.545442 & -0.000028 \\
$\mathrm{C}$ & 4.715915 & -0.437284 & -0.000036 \\
$\mathrm{H}$ & 3.985687 & 1.595687 & -0.000035 \\
$\mathrm{C}$ & 3.026194 & -2.141615 & -0.000009 \\
$\mathrm{H}$ & 1.074291 & -1.347039 & 0.000010 \\
$\mathrm{C}$ & 4.370717 & -1.799301 & -0.000028 \\
$\mathrm{H}$ & 5.761903 & -0.146054 & -0.000048 \\
$\mathrm{H}$ & 2.667160 & -3.164020 & -0.000001 \\
$\mathrm{H}$ & 5.127321 & -2.574796 & -0.000035 \\
$\mathrm{~N}$ & 0.042246 & 0.301948 & -0.000006 \\
$\mathrm{~N}$ & -2.099961 & -1.297110 & 0.000104 \\
$\mathrm{~N}$ & 2.097631 & -1.169169 & -0.000005
\end{tabular}

TerpyH $^{+}$(center)

11

C $\quad-1.201341 \quad 0.968089 \quad 0.000000$ 


$\begin{array}{crrr}\mathrm{C} & 1.201274 & 0.968604 & 0.000000 \\ \mathrm{C} & 1.216205 & 2.363572 & 0.000000 \\ \mathrm{C} & -0.000456 & 3.054450 & 0.000000 \\ \mathrm{C} & -1.216763 & 2.363204 & 0.000000 \\ \mathrm{C} & -2.378225 & 0.066477 & 0.000000 \\ \mathrm{C} & -3.058255 & -2.131490 & 0.000000 \\ \mathrm{C} & -4.412289 & -1.760780 & 0.000000 \\ \mathrm{C} & -4.733463 & -0.401616 & 0.000000 \\ \mathrm{C} & -3.695835 & 0.537758 & 0.000000 \\ \mathrm{H} & 2.153303 & 2.904388 & 0.000000 \\ \mathrm{H} & -0.000598 & 4.140306 & 0.000000 \\ \mathrm{H} & -2.154095 & 2.903588 & 0.000000 \\ \mathrm{H} & -2.772225 & -3.180036 & 0.000000 \\ \mathrm{H} & -5.185491 & -2.521677 & 0.000000 \\ \mathrm{H} & -5.768712 & -0.075859 & 0.000000 \\ \mathrm{H} & -3.922425 & 1.597660 & 0.000000 \\ \mathrm{C} & 2.378279 & 0.067116 & 0.000000 \\ \mathrm{C} & 3.695837 & 0.538652 & 0.000000 \\ \mathrm{C} & 4.733588 & -0.400522 & 0.000000 \\ \mathrm{H} & 3.922158 & 1.598623 & 0.000000 \\ \mathrm{C} & 3.058637 & -2.130705 & 0.000000 \\ \mathrm{C} & 4.412561 & -1.759752 & 0.000000 \\ \mathrm{H} & 5.768808 & -0.074687 & 0.000000 \\ \mathrm{H} & 2.772668 & -3.179248 & 0.000000 \\ \mathrm{H} & 5.185916 & -2.520507 & 0.000000 \\ \mathrm{~N} & 0.000000 & 0.348370 & 0.000000 \\ \mathrm{~N} & -2.063830 & -1.243503 & 0.000000 \\ \mathrm{~N} & 2.064076 & -1.242816 & 0.000000 \\ \mathrm{H} & 0.000453 & -0.685250 & 0.000000 \\ & & & \\ \mathrm{Py} & & & \\ 01 & & & \\ \mathrm{C} & 1.213403 & 0.654701 & 0.000000 \\ \mathrm{C} & 0.000000 & 1.355398 & 0.000000 \\ \mathrm{C} & -1.225199 & -0.580456 & 0.000000 \\ \mathrm{C} & -0.073171 & -1.376384 & 0.000000 \\ \mathrm{C} & 1.175866 & -0.744104 & 0.000000 \\ \mathrm{H} & 2.155754 & 1.193895 & 0.000000 \\ \mathrm{H} & -0.001707 & 2.444214 & 0.000000 \\ \mathrm{H} & -0.156370 & -2.458954 & 0.000000 \\ \mathrm{H} & 2.093238 & -1.325876 & 0.000000 \\ \mathrm{~N} & -1.203618 & 0.762156 & 0.000000 \\ \mathrm{H} & -2.210986 & -1.043302 & 0.000000 \\ & & & \\ \mathrm{PyH} \mathrm{H}^{+} & & & \\ 1 \mathrm{1} & & & \end{array}$



$\begin{array}{llll}\text { C } & 1.214967 & -0.718058 & 0.000000\end{array}$
$\begin{array}{llll}\text { C } & 1.191927 & 0.669222 & 0.000000\end{array}$
$\begin{array}{llll}\text { C } & -1.192198 & 0.668901 & 0.000000\end{array}$
$\begin{array}{llll}\text { C } & -1.214945 & -0.718070 & 0.000000\end{array}$
$\begin{array}{llll}\text { C } & 0.000228 & -1.419878 & 0.000000\end{array}$
$\begin{array}{llll}\mathrm{H} & 2.167438 & -1.235857 & 0.000000\end{array}$
$\mathrm{H} \quad 2.083608 \quad 1.285280 \quad 0.000000$
$\mathrm{H} \quad-2.167125 \quad-1.236394 \quad 0.000000$
$\begin{array}{llll}\mathrm{H} & 0.000069 & -2.505609 & 0.000000\end{array}$
$\begin{array}{llll}\mathrm{N} & 0.000000 & 1.312046 & 0.000000\end{array}$
$\begin{array}{llll}\mathrm{H} & -2.083598 & 1.285383 & 0.000000\end{array}$
$\mathrm{H} \quad-0.000263 \quad 2.330181 \quad 0.00000$ 


\section{REFERENCES.}

(1) Spek, A. L. Single-crystal structure validation with the program PLATON. J. Appl. Cryst. 2003, 36, 7-13.

(2) Janiak, C. A critical account on pi-pi stacking in metal complexes with aromatic nitrogencontaining ligands. J. Chem. Soc., Dalton Trans. 2000, 3885-3896.

(3) Steiner, T. C-H-O Hydrogen Bonding in Crystals. Crystallogr. Rev. 1996, 6, 1-51.

(4) Steiner, T. The Hydrogen Bond in the Solid State. Angew. Chem. Int. Ed. 2002, 41, 48-76.

(5) Zhan, C-G.; Dixon, D. A. Absolute Hydration Free Energy of the Proton from First-Principles Electronic Structure Calculations. J. Phys. Chem. A 2001, 105, $11534-11540$.

(6) Camaioni D.M.; Schwerdtfeger, C.A. Comment on "Accurate Experimental Values for the Free Energies of Hydration of $\mathrm{H}^{+}, \mathrm{OH}^{-}$, and $\mathrm{H}_{3} \mathrm{O}^{+}$". J. Phys. Chem. A 2005, 109, 10795-10797.

(7) Hunter, E. P. L.; Lias, S. G. Evaluated Gas Phase Basicities and Proton Affinities of Molecules: An Update. J. Phys. Chem. Ref. Data, 1998, 27, 413-656.

(8) Haynes, W. M. CRC Handbook of Chemistry and Physics, 91 ${ }^{\text {st }}$ ed.; CRC Press (Taylor \& Francis Group): Boca Raton, FL, 2010.

(9) Krumholz, P. Structural Studies on Polynuclear Pyridine Compounds. J. Am. Chem. Soc. 1951, 73, 3487-3492. 